\title{
Ni-Catalyzed Reductive Aryl-Acylation of Alkenes toward Carbonyl-Containing Oxindoles
}

\author{
Sheng $\mathrm{Xu},{ }^{+}$Kuai Wang, ${ }^{+}$Wangqing Kong* \\ The Center for Precision Synthesis (CPS), Institute for Advanced Studies (IAS), Wuhan University, \\ Wuhan 430072, People's Republic of China \\ Email: wqkong@whu.edu.cn
}




\section{Table of Contents}

1. General information S3

2. General procedures $\quad$ S4

3. Optimization of reaction conditions $\quad$ S6

4. Control experiments $\quad$ S9

5. Characterization data of products $\quad$ S13

6. Copies of the ${ }^{1} \mathrm{H},{ }^{19} \mathrm{~F}$ and ${ }^{13} \mathrm{C}$ NMR spectra $\quad \mathrm{S} 34$

$\begin{array}{ll}\text { 7. References } & \text { S69 }\end{array}$ 


\section{General Information}

${ }^{1} \mathrm{H}$ and ${ }^{13} \mathrm{C}$ NMR data were recorded with Bruker ADVANCE III (400 MHz) or JNMECZ400S/L1 (400 MHz) spectrometers. Chemical shifts are given in ppm. The spectra are calibrated to the residual ${ }^{1} \mathrm{H}$ and ${ }^{13} \mathrm{C}$ signals of the solvents. Multiplicities are abbreviated as follows: singlet $(\mathrm{s})$, doublet $(\mathrm{d})$, triplet $(\mathrm{t})$, quartet $(\mathrm{q})$, doublet-doublet $(\mathrm{dd})$, quintet (quint), septet (sept), multiplet (m), and broad (b). ${ }^{19} \mathrm{~F}$ NMR spectra were recorded using $\mathrm{CFCl}_{3}$ as internal standard. Gas chromatography were determined with a SHIMADZU Nexis GC 2030 gas chromatography instrument with a FID detector. High-resolution mass spectra (HRMS) were recorded on DIONEX UltiMate 3000 \& Bruker Compact TOF mass spectrometer. Enantiomeric excesses were determined with a SHIMADZU LC-20ADXR system using chiral stationary phase columns (DAICEL) by comparing the samples with the corresponding racemic samples. Column and elution details were specified in each entry.

Materials and Methods: Unless otherwise stated, starting materials were purchased from commercial suppliers (Adamas-beta ${ }^{\circledR}$, Alfa, Aldrich and so on). Mn powder (Alfa Aesar140+325 mesh, 99.6\% metals basis). All reactions dealing with air- or moisture-sensitive compounds were performed in the argon-filled glove box or by standard Schlenk techniques in oven-dried reaction vessels under argon atmosphere. Solvents were purchased in HPLC quality, degassed by purging thoroughly with argon and dried over activated molecular sieves of appropriate size. More sensitive compounds were stored in a desiccator or in a glove-box if required. Reactions were monitored by thin layer chromatography (TLC) using glass $0.25 \mathrm{~mm}$ silica gel plates. Compounds were visualized by UV-light at $254 \mathrm{~nm}$ and by dipping the plates in an aqueous potassium permanganate solution followed by heating. Flash column chromatography was performed over silica gel (200-400 mesh). 


\section{General Procedure}

\subsection{General Procedure for Alkyl lodides:}

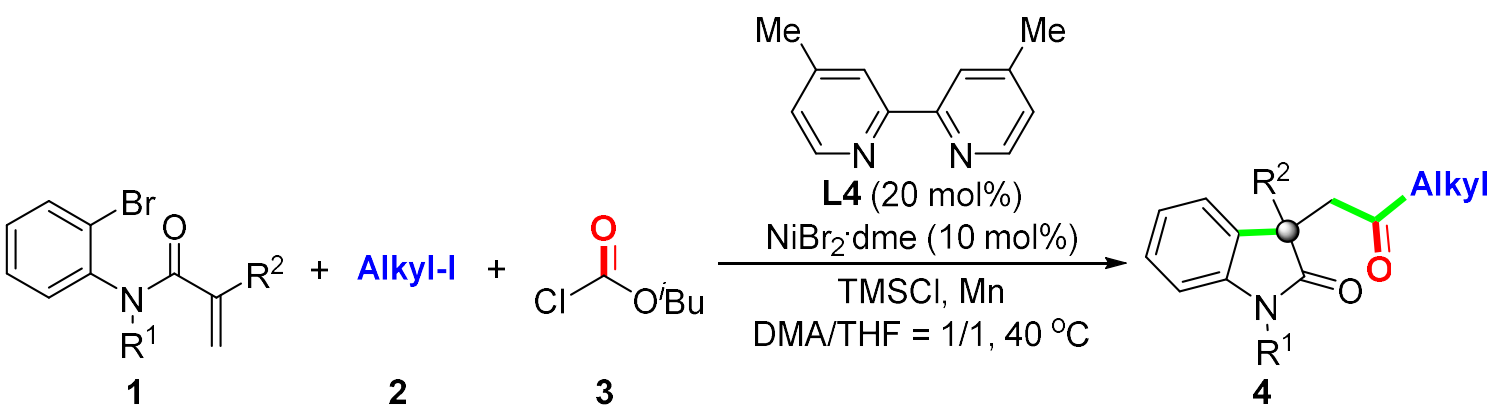

An oven-dried seal-tube equipped with a PTFE-coated stir bar was charged with $\mathrm{NiBr}_{2}$. DME (10 mol\%), L4 (20 mol\%), Mn0 (4 equiv), substrate 1 (0.2 mmol), alkyl iodide 2 (3 equiv), isobutyl chloroformate 3 (3 equiv), TMSCI (0.5 equiv), anhydrous DMA ( $1 \mathrm{~mL})$ and THF (1 mL). The seal-tube was sealed and removed from the glovebox. Then the reaction was stirred at $40{ }^{\circ} \mathrm{C}$ until the reaction was complete (monitored by TLC). The resulting mixture was quenched with saturated $\mathrm{NH}_{4} \mathrm{Cl}$ solution $(5 \mathrm{~mL})$ and further diluted with water $(10 \mathrm{~mL})$. The aqueous layer was extracted with EtOAc and the combined organic layers were washed with brine, dried over anhydrous $\mathrm{Na}_{2} \mathrm{SO}_{4}$, filtered, and concentrated under vacuum. The residue was purified by chromatography on silica gel, eluting with PE/EtOAc (10/1 4/1) to afford the desired oxindole 4.

\subsection{General Procedure for Benzyl Chlorides:}

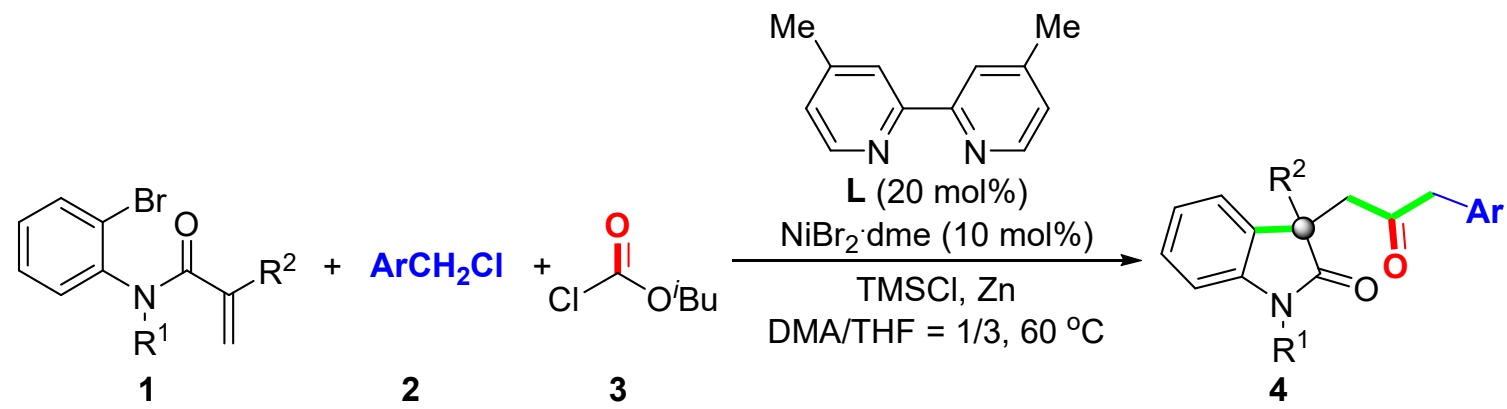

An oven-dried seal-tube equipped with a PTFE-coated stir bar was charged with $\mathrm{NiBr}_{2} \cdot \mathrm{DME}$ (10 mol\%), L4 (20 mol\%), Zn (4 equiv), substrate 1 (0.2 mmol), benzyl chloride 2 (3 equiv), isobutyl chloroformate 3 (3 equiv), TMSCI ( 0.5 equiv), anhydrous 
DMA $(0.5 \mathrm{~mL})$ and THF $(1.5 \mathrm{~mL})$. The seal-tube was sealed and removed from the glovebox. Then the reaction was stirred at $60{ }^{\circ} \mathrm{C}$ until the reaction was complete (monitored by TLC). The resulting mixture was quenched with saturated $\mathrm{NH}_{4} \mathrm{Cl}$ solution (5 $\mathrm{mL}$ ) and further diluted with water $(10 \mathrm{~mL})$. The aqueous layer was extracted with EtOAc and the combined organic layers were washed with brine, dried over anhydrous $\mathrm{Na}_{2} \mathrm{SO}_{4}$, filtered, and concentrated under vacuum. The residue was purified by chromatography on silica gel, eluting with PE/EtOAc (10/1 2/1) to afford the desired oxindole 4.

\subsection{Procedure for $1.0 \mathrm{mmol}$ scale reaction.}

An oven-dried seal-tube $(25 \mathrm{~mL})$ equipped with a PTFE-coated stir bar was charged with $\mathrm{NiBr}_{2}$. DME (30.9 mg, $0.1 \mathrm{mmol}, 0.1$ equiv), L4 (37.3 mg, $0.2 \mathrm{mmol}, 0.2$ equiv), $\mathrm{Mn}^{0}$ (220.0 mg, $4 \mathrm{mmol}, 4$ equiv), substrate 1a $(253.7 \mathrm{mg}, 1 \mathrm{mmol})$, alkyl iodide 2a $(0.4 \mathrm{~mL}, 3$ mmol, 3 equiv), isobutyl chloroformate $3 a(0.4 \mathrm{~mL}, 3 \mathrm{mmol}, 3$ equiv), TMSCl $(0.05 \mathrm{~mL}, 0.5$ mmol, 0.5 equiv), anhydrous DMA ( $5 \mathrm{~mL})$ and THF $(5 \mathrm{~mL})$. The seal-tube was sealed and removed from the glovebox. Then the reaction was stirred at $40{ }^{\circ} \mathrm{C}$ until the reaction was complete (monitored by TLC). The resulting mixture was quenched with saturated $\mathrm{NH}_{4} \mathrm{Cl}$ solution $(10 \mathrm{~mL})$ and further diluted with water $(30 \mathrm{~mL})$. The aqueous layer was extracted with EtOAc and the combined organic layers were washed with brine, dried over anhydrous $\mathrm{Na}_{2} \mathrm{SO}_{4}$, filtered, and concentrated under vacuum. The residue was purified by chromatography on silica gel, eluting with PE/EtOAc (10/1 4/1) to afford the desired oxindole 4aa as colorless oil (185.3 mg, $65 \%$ yield), 


\section{Optimization of reaction conditions}

Table S3-1 Optimization reaction conditions (solvent screening)

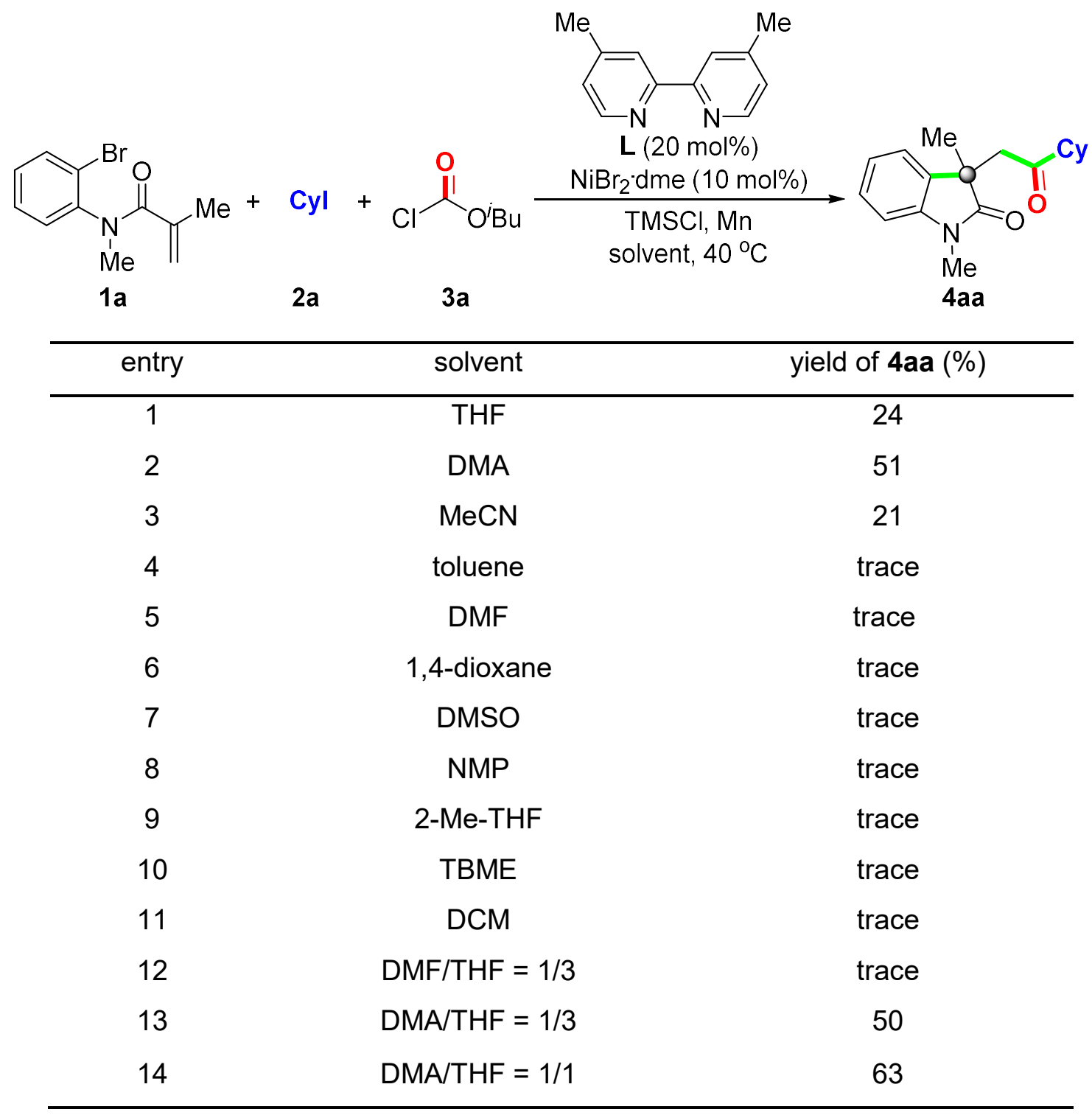


Table S3-2 Optimization reaction conditions ( $\mathrm{Ni}$ sources screening)

3
1


Table S3-3 Optimization of reaction conditions for the enantioselective synthesis of oxindole $4 a a$

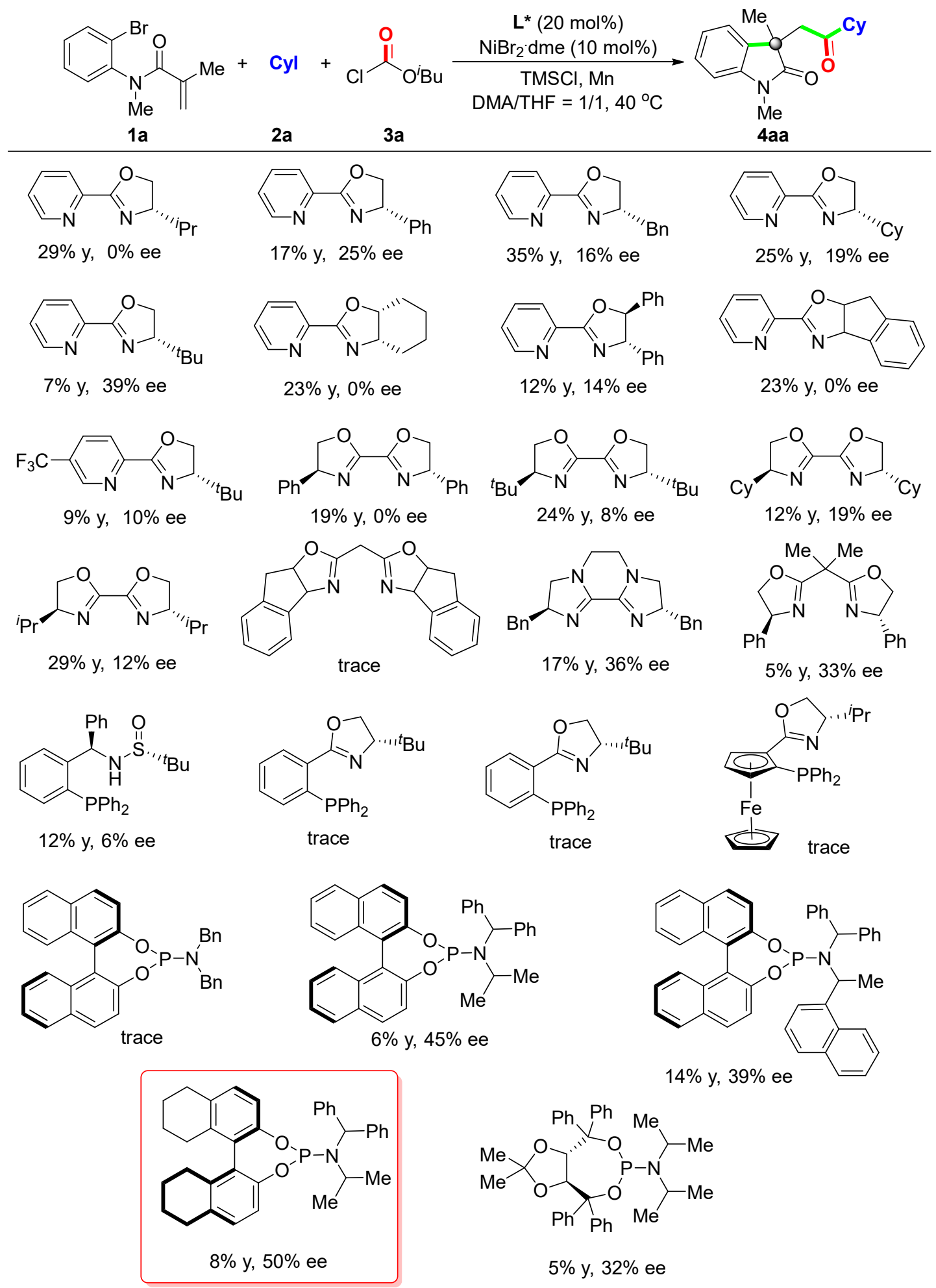




\section{Control experiments}

\subsection{Stoichiometric reaction of $\sigma$-alkyl-Ni(II) 5 with $2 a$ and $3 a$}

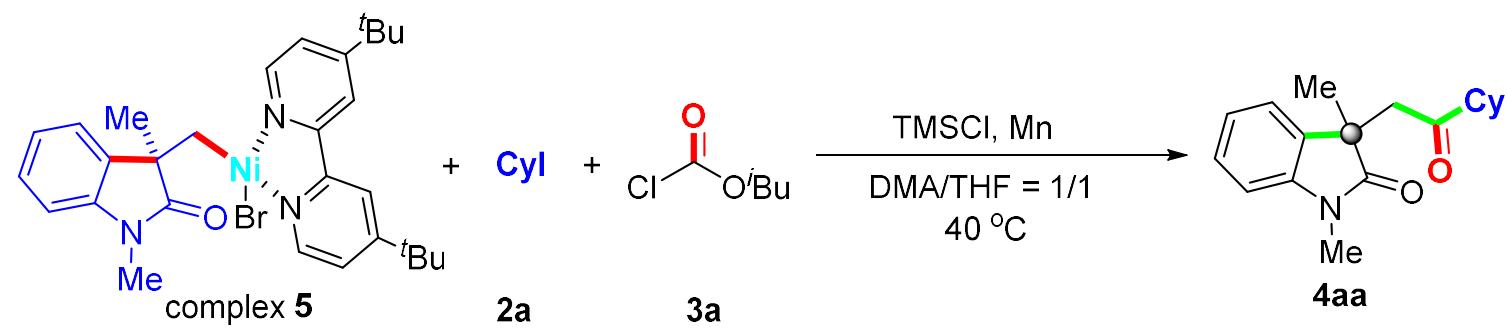

Experimental procedure: An oven-dried seal-tube equipped with a PTFE-coated stir bar was charged with $2 \mathrm{a}(0.15 \mathrm{mmol}, 31.4 \mathrm{mg}), 3 \mathrm{a}(0.15 \mathrm{mmol}, 20.4 \mathrm{mg}), \mathrm{TMSCl}(0.05 \mathrm{mmol}$, $5.4 \mathrm{mg}), \mathrm{Mn}^{0}(0.2 \mathrm{mmol}, 11.0 \mathrm{mg})$ and complex $5(0.05 \mathrm{mmol}, 29.1 \mathrm{mg})$ in anhydrous DMA $(0.5 \mathrm{~mL})$ and THF $(0.5 \mathrm{~mL})$. The seal-tube was sealed and removed from the glovebox. Then the reaction was stirred at $40{ }^{\circ} \mathrm{C}$ until the reaction was complete (monitored by TLC). The resulting mixture was quenched with saturated $\mathrm{NH}_{4} \mathrm{Cl}$ solution $(5 \mathrm{~mL})$ and further diluted with water $(10 \mathrm{~mL})$. The aqueous layer was extracted with EtOAc and the combined organic layers were washed with brine, dried over anhydrous $\mathrm{Na}_{2} \mathrm{SO}_{4}$, filtered, and concentrated under vacuum. The residue was purified by chromatography on silica gel, eluting with PE/EtOAc (10/1 4/1) to afford the desired oxindole 4aa in 36\% yield.

Conclusion: The control experiment without nickel-catalyst did not consume the aryl bromide 1a, suggesting that direct insertion of $\mathrm{Mn}^{0}$ into aryl bromide $1 \mathrm{a}$ is not likely. Taking together, these results indicate that $\sigma$-alkyl-Ni" species $\mathbf{5}$ serves as the key intermediate in the catalytic cycle. 


\subsection{Aryl-acylation reaction with a radical-clock substrate $2 p$}

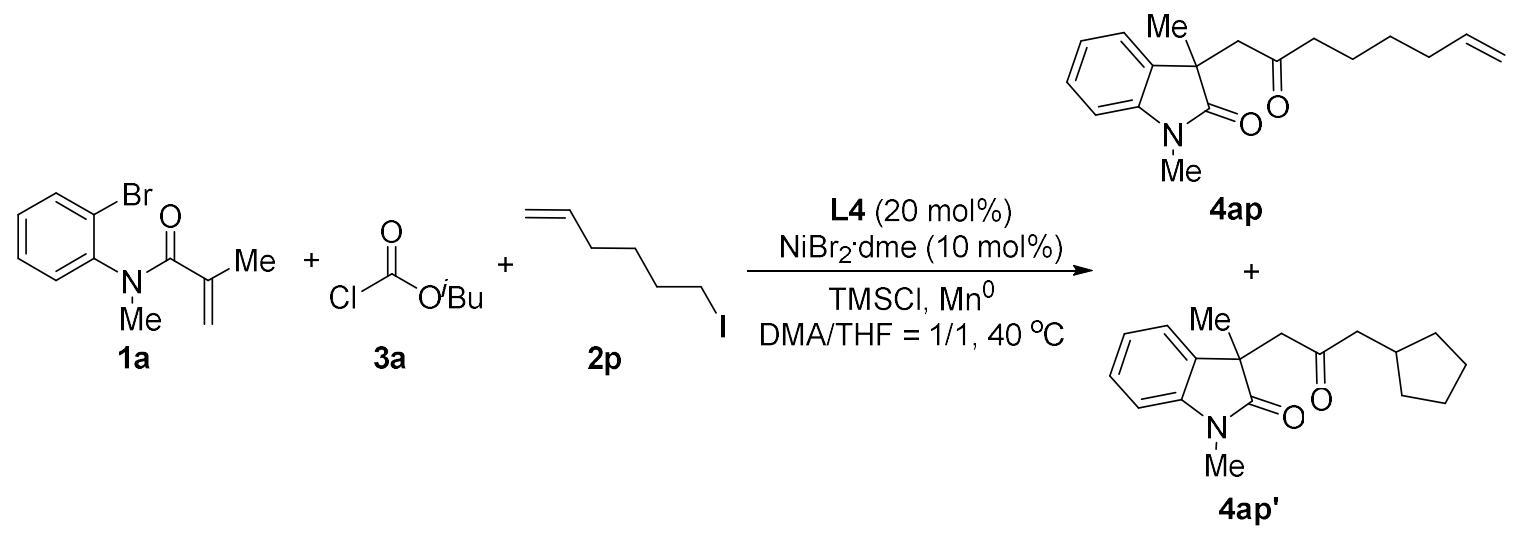

Experimental procedure: An oven-dried seal-tube equipped with a PTFE-coated stir bar was charged with $\mathrm{NiBr}_{2} \cdot \mathrm{DME}(0.01 \mathrm{mmol}, 3.1 \mathrm{mg}), \mathbf{L} 4(0.02 \mathrm{mmol}, 3.7 \mathrm{mg}), 1 \mathrm{a}(0.1 \mathrm{mmol}$, $25.3 \mathrm{mg}$ ), 2 p (0.3 mmol, $63.4 \mathrm{mg}), 3 \mathbf{a}(0.3 \mathrm{mmol}, 40.8 \mathrm{mg}), \mathrm{TMSCl}(0.05 \mathrm{mmol}, 5.4 \mathrm{mg})$, $\mathrm{Mn}^{0}(0.4 \mathrm{mmol}, 22.0 \mathrm{mg})$ in anhydrous DMA $(1 \mathrm{~mL})$ and THF $(1 \mathrm{~mL})$. The seal-tube was sealed and removed from the glovebox. Then the reaction was stirred at $40{ }^{\circ} \mathrm{C}$ until the reaction was complete (monitored by TLC). The resulting mixture was quenched with satuated $\mathrm{NH}_{4} \mathrm{Cl}$ solution $(5 \mathrm{~mL})$ and further diluted with water $(10 \mathrm{~mL})$. The aqueous layer was extracted with EtOAc and the combined organic layers were washed with brine, dried over anhydrous $\mathrm{Na}_{2} \mathrm{SO}_{4}$, filtered, and concentrated under vacuum. The residue was purified by chromatography on silica gel, eluting with PE/EtOAc (10/1 5/1) to afford a 1:1 mixture of the two products 4ap and 4ap'. The overall yield is $60 \%$. The ratio was determined according to the ${ }^{1} \mathrm{H}$ NMR spectrum. ${ }^{1} \mathrm{H}$ NMR $\left(400 \mathrm{MHz}, \mathrm{CDCl}_{3}\right) \delta 7.27-7.20$ $(\mathrm{m}, 2 \mathrm{H}), 7.16-7.09(\mathrm{~m}, 2 \mathrm{H}), 7.04-6.94(\mathrm{~m}, 2 \mathrm{H}), 6.89-6.82(\mathrm{~m}, 2 \mathrm{H})$, 5.79-5.64 (m, 1H), 4.99$4.86(\mathrm{~m}, 2 \mathrm{H}), 3.26(\mathrm{~s}, 6 \mathrm{H}), 3.06(\mathrm{~s}, 3 \mathrm{H}), 3.05(\mathrm{~s}, 3 \mathrm{H}), 2.35-2.13(\mathrm{~m}, 4 \mathrm{H}), 2.35-2.13(\mathrm{~m}, 4 \mathrm{H})$, 1.99-1.90 (m, 2H), 1.62-1.57 (m, 2H), 1.54-1.36 (m, 5H), $1.32(\mathrm{~s}, 6 \mathrm{H}), 1.27-1.18(\mathrm{~s}, 3 \mathrm{H})$; HRMS: (ESI) calcd for $\mathrm{C}_{18} \mathrm{H}_{23} \mathrm{NNaO}_{2}{ }^{+}[\mathrm{M}+\mathrm{Na}]^{+} 308.1621$; found 308.1596 .

Conclusion: This result confirm the involvement of alkyl radical in this transformation. 


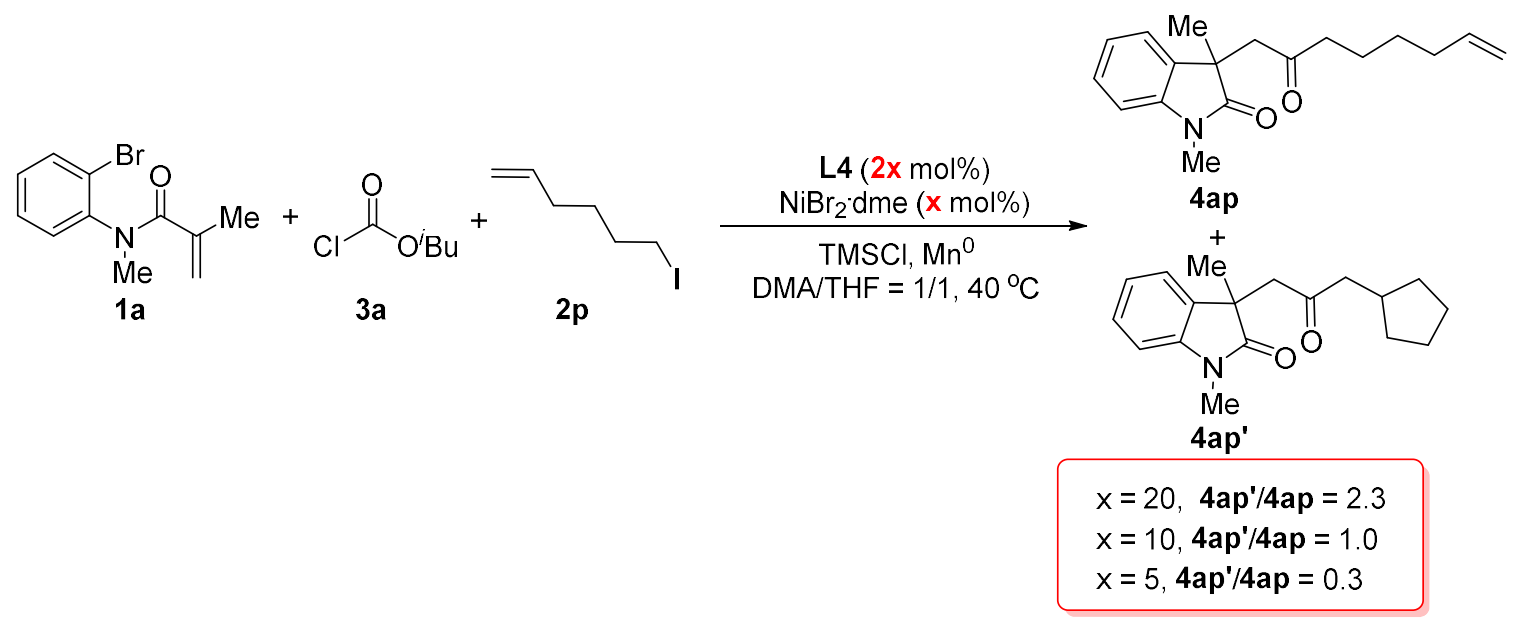

Conclusion: A linear relationship between the Ni-catalyst loading and the product ratio of 4ap and 4ap' was observed, supporting that a radical chain mechanism instead of cagebound oxidative addition. 


\subsection{Aryl-alkylation reaction with $2 a$}<smiles>C=C(C)C(=O)N(C)c1ccccc1Br</smiles>

$1 \mathrm{a}$

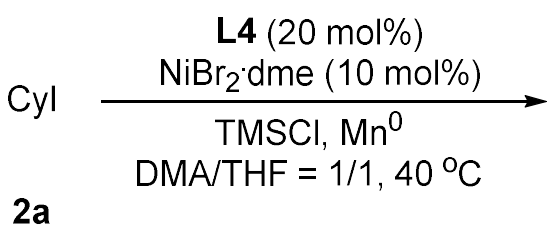

$2 \mathrm{a}$<smiles>CN1C(=O)C(C)(CC2CCCCC2)c2ccccc21</smiles>

6aa $(46 \%)$

Experimental procedure: An oven-dried seal-tube equipped with a PTFE-coated stir bar was charged with $\mathrm{NiBr}_{2}$. DME (0.01 mmol, $\left.3.1 \mathrm{mg}\right)$, L4 (0.02 mmol, $\left.3.7 \mathrm{mg}\right), 1 \mathrm{a}(0.1 \mathrm{mmol}$, $25.3 \mathrm{mg}$ ), 2a (0.3 mmol, $63.4 \mathrm{mg}), \mathrm{TMSCl}$ (0.05 mmol, $5.4 \mathrm{mg}), \mathrm{Mn}^{0}$ (0.4 mmol, $\left.22.0 \mathrm{mg}\right)$ in anhydrous DMA $(1 \mathrm{~mL})$ and THF $(1 \mathrm{~mL})$. The seal-tube was sealed and removed from the glovebox. Then the reaction was stirred at $40{ }^{\circ} \mathrm{C}$ until the reaction was complete (monitored by TLC). The resulting mixture was quenched with satuated $\mathrm{NH}_{4} \mathrm{Cl}$ solution (5 $\mathrm{mL}$ ) and further diluted with water $(10 \mathrm{~mL})$. The aqueous layer was extracted with EtOAc and the combined organic layers were washed with brine, dried over anhydrous $\mathrm{Na}_{2} \mathrm{SO}_{4}$, filtered, and concentrated under vacuum. The residue was purified by chromatography on silica gel, eluting with PE/EtOAc (25/1 15/1) to afford the desired oxindole 6aa in 46\% yield. The NMR data matched those reported in the literature. ${ }^{11} \mathrm{H}$ NMR $\left(400 \mathrm{MHz}, \mathrm{CDCl}_{3}\right)$ ס 7.29-7.23 (m, 1H), 7.17-7.14 (m, 1H), 7.08-7.03 (m, 1H), 6.86-6.82 (m, 1H), 3.22 (s, 3H), $1.93(\mathrm{dd}, J=14.0,6.9 \mathrm{~Hz}, 1 \mathrm{H}), 1.72(\mathrm{dd}, J=14.0,5.2 \mathrm{~Hz}, 1 \mathrm{H}), 1.54-1.43(\mathrm{~m}, 3 \mathrm{H}), 1.38-$ $1.33(\mathrm{~m}, 1 \mathrm{H}), 1.31(\mathrm{~s}, 3 \mathrm{H}), 1.23-1.17(\mathrm{~m}, 1 \mathrm{H}), 1.01-0.69(\mathrm{~m}, 6 \mathrm{H})$.

Conclusion: The alkyl radical reacts faster with the acyl species $\mathbf{D}$ than with the $\sigma$-alkyl$\mathrm{Nil}^{1}$ intermediate $\mathrm{A}$, which leads to the selectivity towards dialkyl ketone product 4 , rather than the noncarbonylative product 6 . 


\section{Characterization data of products}

3-(2-cyclohexyl-2-oxoethyl)-1,3-dimethylindolin-2-one (4aa)

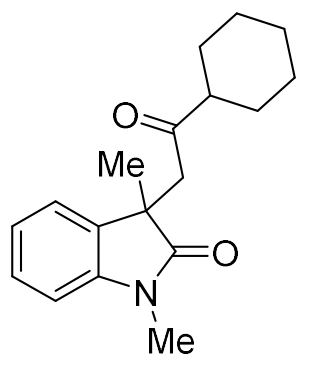

Chemical Formula: $\mathrm{C}_{18} \mathrm{H}_{23} \mathrm{NO}_{2}$

Exact Mass: 285.1729

4aa was prepared according to general procedure 2.1 using $\mathbf{1 a}$ and $\mathbf{2 a}$ and was purified by silica gel column chromatography $(\mathrm{PE} / \mathrm{EtOAc}=10 / 1 \sim 4 / 1)$ to obtain 4aa as colorless oil (35.9 mg, 63\% yield). The NMR data matched those reported in the literature. ${ }^{2}{ }^{1} \mathrm{H}$ NMR $\left(400 \mathrm{MHz}, \mathrm{CDCl}_{3}\right)$ ठ 7.24-7.19 (m, 1H), $7.08(\mathrm{dd}, J=7.4,1.3 \mathrm{~Hz}, 1 \mathrm{H}), 6.99-6.94(\mathrm{~m}, 1 \mathrm{H})$, $6.83(\mathrm{dd}, J=7.8,0.9 \mathrm{~Hz}, 1 \mathrm{H}), 3.25(\mathrm{~d}, J=0.5 \mathrm{~Hz}, 3 \mathrm{H}), 3.14(\mathrm{~d}, J=17.9 \mathrm{~Hz}, 1 \mathrm{H}), 3.06$ (d, $J=17.8 \mathrm{~Hz}, 1 \mathrm{H}), 2.21-2.11(\mathrm{~m}, 1 \mathrm{H}), 1.73-1.65(\mathrm{~m}, 4 \mathrm{H}), 1.62-1.54(\mathrm{~m}, 1 \mathrm{H}), 1.30(\mathrm{~s}, 3 \mathrm{H})$, 1.25-1.03 (m, 5H); ${ }^{13} \mathrm{C}$ NMR (101 MHz, $\left.\mathrm{CDCl}_{3}\right) \delta 210.0,180.5,143.8,133.7,127.7,122.0$, $121.5,108.1,50.4,47.8,45.0,28.2$, 27.9, 26.3, 25.7, 25.5, 25.4, 24.5.

The enantiomeric purity was established by HPLC analysis using a chiral column: AD-H column, $30{ }^{\circ} \mathrm{C}, n$-Hexane/i-Propanol $=95 / 5$ as eluent, $254 \mathrm{~nm}, 1 \mathrm{~mL} / \mathrm{min}$. $\mathrm{tR}=9.0 \mathrm{~min}$ (minor), $10.5 \mathrm{~min}$ (major). 
$\mathrm{mAU}$

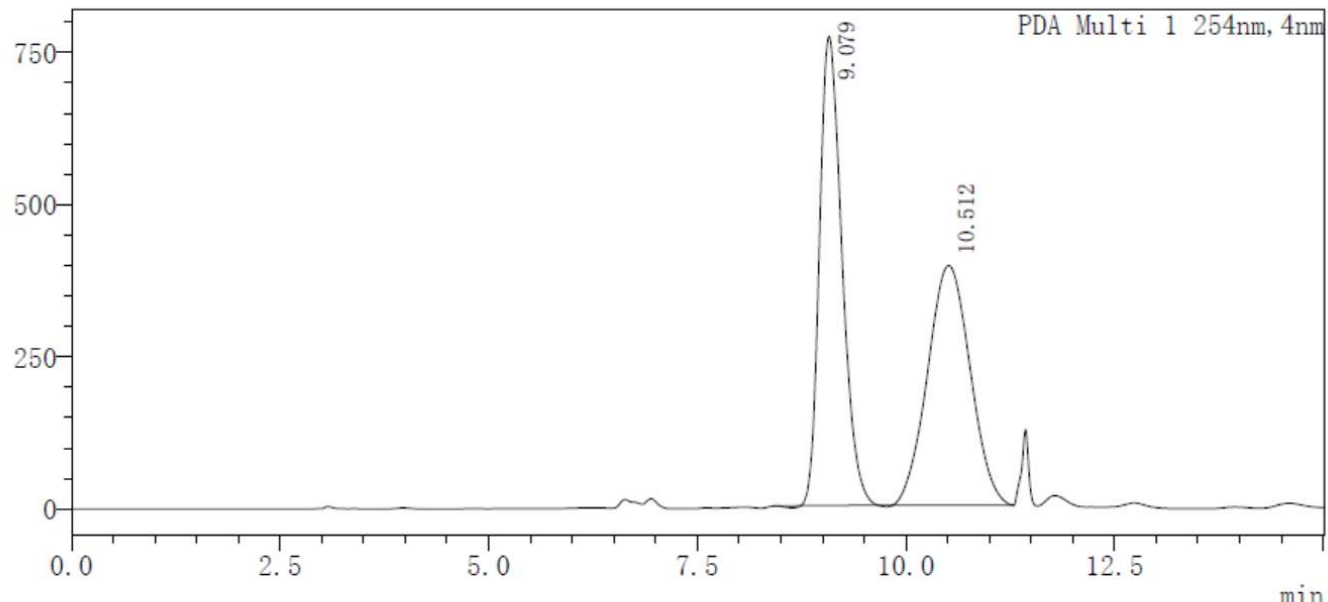

〈峰表〉

\begin{tabular}{|c|c|c|c|c|c|c|c|}
\hline 峰号 & 保留时间 & 面积 & 高度 & 浓度 & 浓度单位 & 标记 & 化合物名 \\
\hline$\$ 1$ & $\$ 9.079$ & \$14444433 & 爪 771294 & 0.000 & & M & \\
\hline 2 & 0.512 & 14006604 & 393751 & 0.000 & & M & \\
\hline 总计 & & 28451037 & 1165046 & & & & \\
\hline $\mathrm{am}$ & & rea & & & & & \\
\hline
\end{tabular}

$\mathrm{mAU}$

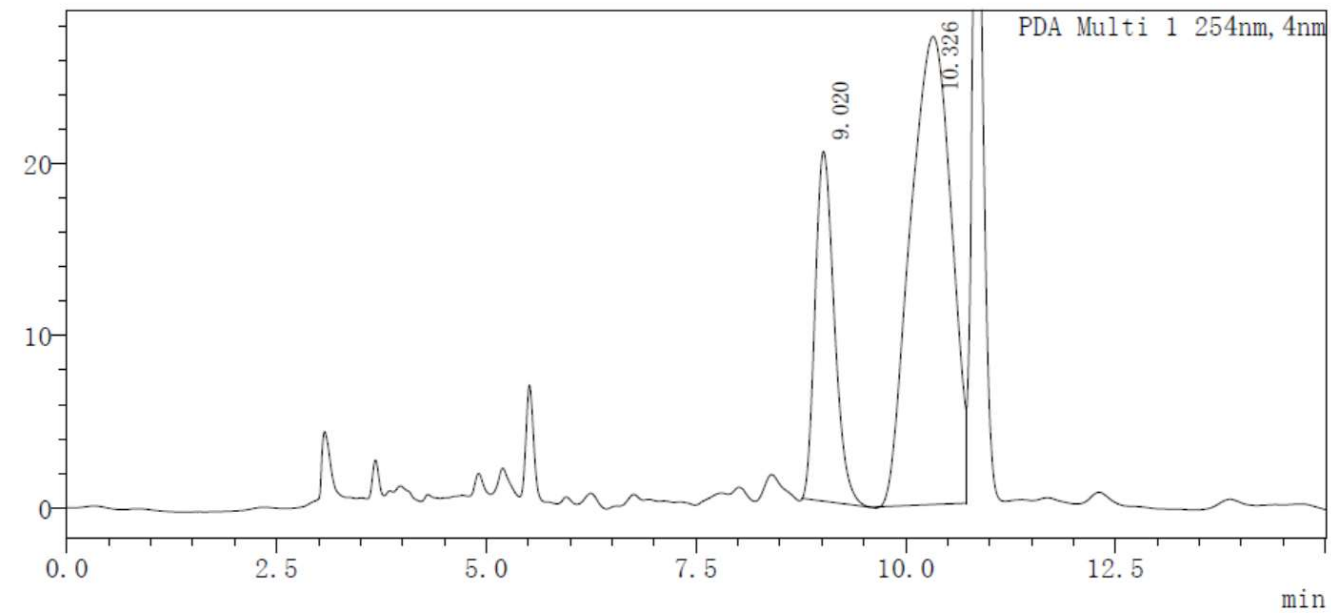

〈峰表〉

\begin{tabular}{|c|c|c|c|c|c|c|c|}
\hline 峰号 & 保留时间 & 面积 & 高度 & 浓度 & 浓度单位 & 标记 & 化合物名 \\
\hline$\$ 1$ & $\triangle 9.020$ & $\wedge 323908$ & 木 20340 & 0.000 & & M & \\
\hline 2 & 10.326 & 922853 & 27226 & 0.000 & & M & \\
\hline 总计 & & 1246761 & 47566 & & & & \\
\hline
\end{tabular}

retention time 
3-(2-cyclohexyl-2-oxoethyl)-1,3,5-trimethylindolin-2-one (4ba)<smiles>Cc1ccc2c(c1)C(C)(CC(=O)C1CCCCC1)C(=O)N2C</smiles>

Chemical Formula: $\mathrm{C}_{19} \mathrm{H}_{25} \mathrm{NO}_{2}$

Exact Mass: 299.1885

4ba was prepared according to general procedure 2.1 using $\mathbf{1 b}$ and $\mathbf{2 a}$ and was purified by silica gel column chromatography $(\mathrm{PE} / \mathrm{EtOAc}=10 / 1 \sim 4 / 1)$ to obtain $\mathbf{4 b a}$ as colorless oil (35.9 mg, 60\% yield). ${ }^{1} \mathrm{H}$ NMR (400 MHz, $\left.\mathrm{CDCl}_{3}\right) \delta$ 7.05-7.00 (m, 1H), 6.93-6.88 (m, 1H), $6.73(\mathrm{~d}, J=7.8 \mathrm{~Hz}, 1 \mathrm{H}), 3.24(\mathrm{~s}, 3 \mathrm{H}), 3.12(\mathrm{~d}, J=17.8 \mathrm{~Hz}, 1 \mathrm{H}), 3.06(\mathrm{~d}, J=17.8 \mathrm{~Hz}, 1 \mathrm{H})$, $2.30(\mathrm{~d}, J=0.7 \mathrm{~Hz}, 3 \mathrm{H}), 2.23-2.11(\mathrm{~m}, 1 \mathrm{H}), 1.73-1.67(\mathrm{~m}, 4 \mathrm{H}), 1.63-1.56(\mathrm{~m}, 1 \mathrm{H}), 1.30(\mathrm{~s}$, $3 \mathrm{H}), 1.25-1.06(\mathrm{~m}, 5 \mathrm{H}) ;{ }^{13} \mathrm{C}$ NMR (101 MHz, $\left.\mathrm{CDCl}_{3}\right) \delta$ 210.1, 180.5, 141.4, 133.7, 131.5, 127.9, 122.5, 107.8, 50.4, 47.7, 45.1, 28.2, 28.0, 26.4, 25.7, 25.6, 25.5, 24.6, 21.1; HRMS: (ESI) calcd for $\mathrm{C}_{19} \mathrm{H}_{25} \mathrm{NNaO}_{2}{ }^{+}[\mathrm{M}+\mathrm{Na}]^{+} 322.1777$; found 322.1751 .

3-(2-cyclohexyl-2-oxoethyl)-5-methoxy-1,3-dimethylindolin-2-one (4ca)<smiles>COc1ccc2c(c1)C(C)(CC(=O)C1CCCCC1)C(=O)N2C</smiles>

Chemical Formula: $\mathrm{C}_{19} \mathrm{H}_{25} \mathrm{NO}_{3}$

Exact Mass: 315.1834

4ca was prepared according to general procedure 2.1 using $\mathbf{1 c}$ and $\mathbf{2 a}$ and was purified by silica gel column chromatography $(\mathrm{PE} / \mathrm{EtOAc}=10 / 1 \sim 2 / 1)$ to obtain 4 ca as colorless oil (42.4 mg, 67\% yield). The NMR data matched those reported in the literature. ${ }^{2}{ }^{1} \mathrm{H}$ NMR $\left(400 \mathrm{MHz}, \mathrm{CDCl}_{3}\right) \delta 6.75$ (d, J = $1.5 \mathrm{~Hz}, 2 \mathrm{H}$ ), 6.74 (dd, J = 1.5, $0.8 \mathrm{~Hz}, 1 \mathrm{H}$ ), $3.76(\mathrm{~s}, 3 \mathrm{H})$, 3.24 (s, 3H), 3.12 (d, J = $17.9 \mathrm{~Hz}, 1 \mathrm{H}), 3.08(\mathrm{~d}, J=17.9 \mathrm{~Hz}, 1 \mathrm{H}), 2.24-2.14(\mathrm{~m}, 1 \mathrm{H}), 1.76-$ $1.67(\mathrm{~m}, 4 \mathrm{H}), 1.65-1.56(\mathrm{~m}, 1 \mathrm{H}), 1.31(\mathrm{~s}, 3 \mathrm{H}), 1.28-1.03(\mathrm{~m}, 5 \mathrm{H}) ;{ }^{13} \mathrm{C}$ NMR $(101 \mathrm{MHz}$, 
$\left.\mathrm{CDCl}_{3}\right) \delta 210.1,180.3,155.8,137.5,135.3,111.4,109.9,108.3,55.9,50.5,47.8,45.6$, 28.3, 28.1, 26.6, 25.8, 25.7, 25.6, 24.7.

3-(2-cyclohexyl-2-oxoethyl)-1,3-dimethyl-5-(trifluoromethyl)indolin-2-one (4da)<smiles>CN1C(=O)C(C)(CC(=O)C2CCCCC2)c2cc(C(F)(F)F)ccc21</smiles>

Chemical Formula: $\mathrm{C}_{19} \mathrm{H}_{22} \mathrm{~F}_{3} \mathrm{NO}_{2}$

Exact Mass: 353.1603

4da was prepared according to general procedure 2.1 using $1 \mathbf{d}$ and $\mathbf{2 a}$ and was purified by silica gel column chromatography $(\mathrm{PE} / \mathrm{EtOAc}=10 / 1 \sim 2 / 1)$ to obtain $\mathbf{4 d a}$ as colorless oil (41.0 mg, 58\% yield). ${ }^{1} \mathrm{H}$ NMR (400 MHz, $\left.\mathrm{CDCl}_{3}\right) \delta 7.55-7.51(\mathrm{~m}, 1 \mathrm{H}), 7.30(\mathrm{~d}, \mathrm{~J}=1.9 \mathrm{~Hz}$, $1 \mathrm{H}), 6.92(\mathrm{~d}, J=8.2 \mathrm{~Hz}, 1 \mathrm{H}), 3.30(\mathrm{~s}, 3 \mathrm{H}), 3.21(\mathrm{~d}, J=18.2 \mathrm{~Hz}, 1 \mathrm{H}), 3.15(\mathrm{~d}, J=18.2 \mathrm{~Hz}$, $1 \mathrm{H}), 2.25-2.16(\mathrm{~m}, 1 \mathrm{H}), 1.76-1.67(\mathrm{~m}, 4 \mathrm{H}), 1.64-1.57(\mathrm{~m}, 1 \mathrm{H}), 1.34(\mathrm{~s}, 3 \mathrm{H}), 1.27-1.03(\mathrm{~m}$, $5 \mathrm{H}) ;{ }^{13} \mathrm{C}$ NMR (101 MHz, $\left.\mathrm{CDCl}_{3}\right) \delta 210.1,180.6,147.0,134.5,125.7$ (q, J=4.1 Hz), 124.6 (q, $J=272.7 \mathrm{~Hz}), 124.3(\mathrm{q}, J=32.3 \mathrm{~Hz}), 118.5(\mathrm{q}, J=3.6 \mathrm{~Hz}), 107.9,50.4,48.0$, 45.0, 28.2, 28.1, 26.7, 25.7, 25.6, 25.5, 24.5; ${ }^{19} \mathrm{~F} \mathrm{NMR} \mathrm{(376} \mathrm{MHz,} \mathrm{CDCl}_{3}$ ) $\delta-61.21$; HRMS: (ESI) calcd for $\mathrm{C}_{19} \mathrm{H}_{22} \mathrm{~F}_{3} \mathrm{NNaO}_{2}{ }^{+}[\mathrm{M}+\mathrm{Na}]^{+}$376.1495; found 376.1495.

3-(2-cyclohexyl-2-oxoethyl)-5-fluoro-1,3-dimethylindolin-2-one (4ea)<smiles>CN1C(=O)C(C)(CC(=O)C2CCCCC2)c2cc(F)ccc21</smiles>

Chemical Formula: $\mathrm{C}_{18} \mathrm{H}_{22} \mathrm{FNO}_{2}$

Exact Mass: 303.1635 
4ea was prepared according to general procedure 2.1 using $1 \mathbf{e}$ and $2 \mathrm{a}$ and was purified by silica gel column chromatography $(\mathrm{PE} / \mathrm{EtOAc}=10 / 1 \sim 4 / 1)$ to obtain 4 ea as colorless oil (36.4 mg, 60\% yield). The ${ }^{1} \mathrm{H}$ NMR data matched those reported in the literature. ${ }^{2}{ }^{1} \mathrm{H}$ NMR $\left(400 \mathrm{MHz}, \mathrm{CDCl}_{3}\right) \delta$ 6.97-6.91 (m, 1H), $6.86(\mathrm{dd}, J=7.9,2.6 \mathrm{~Hz}, 1 \mathrm{H}), 6.77$ (dd, $J=8.5$, $4.2 \mathrm{~Hz}, 1 \mathrm{H}), 3.25(\mathrm{~s}, 3 \mathrm{H}), 3.12(\mathrm{~s}, 2 \mathrm{H}), 2.25-2.16(\mathrm{~m}, 1 \mathrm{H}), 1.75-1.69(\mathrm{~m}, 4 \mathrm{H}), 1.65-1.57(\mathrm{~m}$, 1H), 1.32 (s, 3H), 1.26-1.08 (m, 5H); ${ }^{13} \mathrm{C}$ NMR (101 MHz, $\left.\mathrm{CDCl}_{3}\right) \delta$ 209.9, 180.2, 159.1 (d, $J=239.6 \mathrm{~Hz}), 139.7,135.4$ (d, $J=7.8 \mathrm{~Hz}), 113.7$ (d, $J=23.3 \mathrm{~Hz}), 109.8$ (d, $J=24.7 \mathrm{~Hz})$, $108.5(\mathrm{~d}, J=8.1 \mathrm{~Hz}), 50.3,47.7,45.5,28.2,28.0,26.5,25.7,25.5,25.4,24.4 ;{ }^{19} \mathrm{~F}$ NMR (376 $\left.\mathrm{MHz} \mathrm{CDCl}_{3}\right) \delta-121.24$.

3-(2-cyclohexyl-2-oxoethyl)-1,3,6-trimethylindolin-2-one (4fa)<smiles>Cc1ccc2c(c1)N(C)C(=O)C2(C)CC(=O)C1CCCCC1</smiles>

Chemical Formula: $\mathrm{C}_{19} \mathrm{H}_{25} \mathrm{NO}_{2}$

Exact Mass: 299.1885

4fa was prepared according to general procedure 2.1 using $\mathbf{1 f}$ and $\mathbf{2 a}$ and was purified by silica gel column chromatography $(\mathrm{PE} / \mathrm{EtOAc}=10 / 1 \sim 4 / 1)$ to obtain $\mathbf{4 f a}$ as colorless oil (39.5 mg, 66\% yield). ${ }^{1} \mathrm{H}$ NMR $\left(400 \mathrm{MHz}, \mathrm{CDCl}_{3}\right)$ ठ 6.98 (d, J = 7.4 Hz, 1H), 6.82-6.76 (m, $1 \mathrm{H}), 6.70-6.65(\mathrm{~m}, 1 \mathrm{H}), 3.25(\mathrm{~s}, 3 \mathrm{H}), 3.13(\mathrm{~d}, J=17.8 \mathrm{~Hz}, 1 \mathrm{H}), 3.05(\mathrm{~d}, J=17.8 \mathrm{~Hz}, 1 \mathrm{H})$, $2.36(\mathrm{~d}, J=0.7 \mathrm{~Hz}, 3 \mathrm{H}), 2.23-2.12(\mathrm{~m}, 1 \mathrm{H}), 1.78-1.66(\mathrm{~m}, 5 \mathrm{H}), 1.65-1.56(\mathrm{~m}, 1 \mathrm{H}), 1.31$ (s, $3 \mathrm{H}), 1.27-1.08(\mathrm{~m}, 5 \mathrm{H}) ;{ }^{13} \mathrm{C}$ NMR $\left(101 \mathrm{MHz}, \mathrm{CDCl}_{3}\right) \delta$ 210.1, 180.8, 143.8, 137.7, 130.7, 122.5, 121.3, 109.1, 50.4, 47.8, 44.8, 28.2, 27.9, 26.3, 25.7, 25.6, 25.4, 24.6, 21.7; HRMS: (ESI) calcd for $\mathrm{C}_{19} \mathrm{H}_{25} \mathrm{NNaO}_{2}{ }^{+}[\mathrm{M}+\mathrm{Na}]^{+} 322.1777$; found 322.1771 .

3-(2-cyclohexyl-2-oxoethyl)-6-methoxy-1,3-dimethylindolin-2-one (4ga) 
<smiles>COc1ccc2c(c1)N(C)C(=O)C2(C)CC(=O)C1CCCCC1</smiles>

Chemical Formula: $\mathrm{C}_{19} \mathrm{H}_{25} \mathrm{NO}_{3}$

Exact Mass: 315.1834

4ga was prepared according to general procedure 2.1 using $\mathbf{1 g}$ and $\mathbf{2 a}$ and was purified by silica gel column chromatography $(\mathrm{PE} / \mathrm{EtOAc}=10 / 1 \sim 2 / 1)$ to obtain $\mathbf{4 g a}$ as colorless oil (43.5 mg, 69\% yield). The ${ }^{1} \mathrm{H}$ NMR data matched those reported in the literature. ${ }^{2}{ }^{1} \mathrm{H}$ NMR $\left(400 \mathrm{MHz}, \mathrm{CDCl}_{3}\right) \delta 7.00(\mathrm{~d}, J=8.1 \mathrm{~Hz}, 1 \mathrm{H}), 6.48(\mathrm{dd}, J=8.1,2.3 \mathrm{~Hz}, 1 \mathrm{H}), 6.44(\mathrm{~d}, J=$ $2.3 \mathrm{~Hz}, 1 \mathrm{H}), 3.80(\mathrm{~s}, 3 \mathrm{H}), 3.24(\mathrm{~s}, 3 \mathrm{H}), 3.12(\mathrm{~d}, J=17.8 \mathrm{~Hz}, 1 \mathrm{H}), 3.03(\mathrm{~d}, J=17.7 \mathrm{~Hz}, 1 \mathrm{H})$, 2.22-2.13 (m, 1H), 1.75-1.67 (m, 4H), 1.65-1.55 (m, 1H), $1.30(\mathrm{~s}, 3 \mathrm{H}), 1.27-1.02(\mathrm{~m}, 5 \mathrm{H})$; ${ }^{13} \mathrm{C} \mathrm{NMR}\left(101 \mathrm{MHz}, \mathrm{CDCl}_{3}\right) \delta 210.1,181.0,159.9,144.9,125.6,122.1,105.9,96.1,55.35$, $50.5,47.8,44.5,28.1,27.9,26.3,25.5,25.5,25.4,24.5$.

6-chloro-3-(2-cyclohexyl-2-oxoethyl)-1,3-dimethylindolin-2-one (4ha)<smiles>CN1C(=O)C(C)(CC(=O)C2CCCCC2)c2ccc(Cl)cc21</smiles>

Chemical Formula: $\mathrm{C}_{18} \mathrm{H}_{22} \mathrm{CINO}_{2}$

Exact Mass: 319.1339

4ha was prepared according to general procedure 2.1 using $\mathbf{1 h}$ and $\mathbf{2 a}$ and was purified by silica gel column chromatography $(\mathrm{PE} / \mathrm{EtOAc}=10 / 1 \sim 4 / 1)$ to obtain 4 ha as colorless oil (37.0 mg, 58\% yield). ${ }^{1} \mathrm{H}$ NMR $\left(400 \mathrm{MHz}, \mathrm{CDCl}_{3}\right) \delta 7.00$ (d, $\left.J=7.9 \mathrm{~Hz}, 1 \mathrm{H}\right), 6.95$ (dd, $J=$ 7.9, $1.8 \mathrm{~Hz}, 1 \mathrm{H}), 6.85$ (d, J=1.8 Hz, 1H), 3.25 (s, 3H), 3.14 (d, J = $18.0 \mathrm{~Hz}, 1 \mathrm{H}), 3.09$ (d, $J=18.0 \mathrm{~Hz}, 1 \mathrm{H}), 2.24-2.14(\mathrm{~m}, 1 \mathrm{H}), 1.77-1.66(\mathrm{~m}, 4 \mathrm{H}), 1.65-1.57(\mathrm{~m}, 1 \mathrm{H}), 1.30(\mathrm{~s}, 3 \mathrm{H})$, 1.25-1.04 (m, 5H); ${ }^{13} \mathrm{C}$ NMR (101 MHz, $\left.\mathrm{CDCl}_{3}\right) \delta 209.9,180.5,145.1,133.5,132.1,122.3$, 
121.8, 108.9, 50.3, 47.8, 44.8, 28.21, 27.9, 26.5, 25.7, 25.6, 25.4, 24.4; HRMS: (ESI) calcd for $\mathrm{C}_{18} \mathrm{H}_{22} \mathrm{ClNNaO}_{2}{ }^{+}[\mathrm{M}+\mathrm{Na}]^{+} 342.1231$; found 342.1231 .

3-(2-cyclohexyl-2-oxoethyl)-1,3-dimethyl-6-phenylindolin-2-one (4ia)

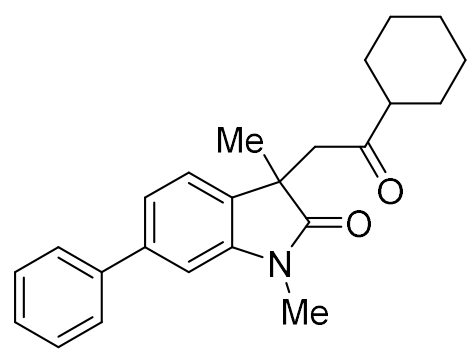

Chemical Formula: $\mathrm{C}_{24} \mathrm{H}_{27} \mathrm{NO}_{2}$

Exact Mass: 361.2042

4ia was prepared according to general procedure 2.1 using 3-( $\mathrm{N}$-methylmethacrylamido)[1,1'-biphenyl]-4-yl trifluoromethanesulfonate $1 \mathbf{i}$ and $2 \mathbf{a}$ and was purified by silica gel column chromatography (PE/EtOAc $=10 / 1 \sim 5 / 1)$ to obtain 4ia as colorless oil $(43.3 \mathrm{mg}$, $60 \%$ yield). ${ }^{1} \mathrm{H}$ NMR $\left(400 \mathrm{MHz}, \mathrm{CDCl}_{3}\right)$ ठ 7.60-7.56 (m, 2H), 7.46-7.41 (m, 2H), 7.37-7.32 $(\mathrm{m}, 1 \mathrm{H}), 7.21-7.14(\mathrm{~m}, 2 \mathrm{H}), 7.04(\mathrm{dd}, J=1.6,0.6 \mathrm{~Hz}, 1 \mathrm{H}), 3.32(\mathrm{~s}, 3 \mathrm{H}), 3.19(\mathrm{~d}, J=17.9$ $\mathrm{Hz}, 1 \mathrm{H}), 3.11(\mathrm{~d}, J=17.9 \mathrm{~Hz}, 1 \mathrm{H}), 2.28-2.14(\mathrm{~m}, 1 \mathrm{H}), 1.75-1.68(\mathrm{~m}, 4 \mathrm{H}), 1.65-1.57(\mathrm{~m}$, $1 \mathrm{H}), 1.36(\mathrm{~s}, 3 \mathrm{H}), 1.27-1.05(\mathrm{~m}, 5 \mathrm{H}) ;{ }^{13} \mathrm{C} \mathrm{NMR}\left(101 \mathrm{MHz}, \mathrm{CDCl}_{3}\right) \delta 210.1,180.7,144.3$, 141.3, 141.2, 132.7, 128.7, 127.3, 127.2, 121.8, 121.1, 107.1, 50.4, 47.8, 45.0, 28.2, 28.0, 26.4, 25.7, 25.6, 25.4, 24.6; HRMS: (ESI) calcd for $\mathrm{C}_{24} \mathrm{H}_{27} \mathrm{NNaO}_{2}{ }^{+}[\mathrm{M}+\mathrm{Na}]^{+} 384.1934$; found 384.1935 .

3-(2-cyclohexyl-2-oxoethyl)-1,3,7-trimethylindolin-2-one (4ja)<smiles>Cc1cccc2c1N(C)C(=O)C2(C)CC(=O)C1CCCCC1</smiles>

Chemical Formula: $\mathrm{C}_{19} \mathrm{H}_{25} \mathrm{NO}_{2}$

Exact Mass: 299.1885 
4ja was prepared according to general procedure 2.1 using $\mathbf{1 j}$ and $\mathbf{2 a}$ and was purified by silica gel column chromatography $(\mathrm{PE} / \mathrm{EtOAc}=10 / 1 \sim 4 / 1)$ to obtain $4 \mathrm{ja}$ as colorless oil (28.1 mg, 47\% yield). 1H NMR (400 MHz, $\left.\mathrm{CDCl}_{3}\right)$ ס 6.97-6.93 (m, 1H), 6.92-6.89 (m, 1H), $6.86(\mathrm{t}, J=7.4 \mathrm{~Hz}, 1 \mathrm{H}), 3.55(\mathrm{~s}, 3 \mathrm{H}), 3.13(\mathrm{~d}, J=17.8 \mathrm{~Hz}, 1 \mathrm{H}), 3.08(\mathrm{~d}, J=17.8 \mathrm{~Hz}, 1 \mathrm{H})$, $2.59(\mathrm{~s}, 3 \mathrm{H}), 2.22-2.11(\mathrm{~m}, 1 \mathrm{H}), 1.75-1.66(\mathrm{~m}, 4 \mathrm{H}), 1.64-1.57(\mathrm{~m}, 1 \mathrm{H}), 1.29(\mathrm{~s}, 3 \mathrm{H}), 1.26-$ $1.05(\mathrm{~m}, 5 \mathrm{H}) ;{ }^{13} \mathrm{C}$ NMR $\left(101 \mathrm{MHz}, \mathrm{CDCl}_{3}\right) \delta$ 210.0, 181.3, 141.6, 134.3, 131.5, 121.9, 119.7, 119.3, 50.4, 48.1, 44.4, 29.7, 28.2, 28.0, 25.7, 25.6, 25.4, 25.1, 19.1; HRMS: (ESI) calcd for $\mathrm{C}_{19} \mathrm{H}_{25} \mathrm{NNaO}_{2}{ }^{+}[\mathrm{M}+\mathrm{Na}]^{+} 322.1777$; found 322.1777 .

3-(2-cyclohexyl-2-oxoethyl)-1,3-dimethyl-1,3-dihydro-2H-benzo[f]indol-2-one (4ka)

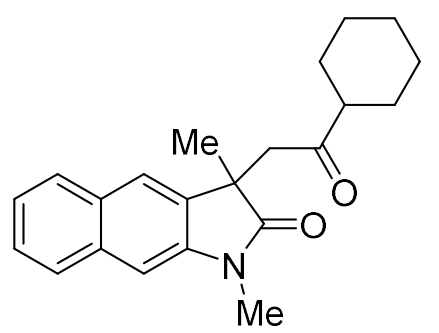

Chemical Formula: $\mathrm{C}_{22} \mathrm{H}_{25} \mathrm{NO}_{2}$

Exact Mass: 335.1885

4ka was prepared according to general procedure 2.1 using 3-( $N$-methylmethacrylamido) naphthalen-2-yl trifluoromethanesulfonate $1 \mathbf{k}$ and $2 \mathrm{a}$ and was purified by silica gel column chromatography $(\mathrm{PE} /$ EtOAc $=10 / 1 \sim 5 / 1)$ to obtain 4ka as colorless oil (45.6 mg, 68\% yield). ${ }^{1} \mathrm{H}$ NMR $\left(400 \mathrm{MHz}, \mathrm{CDCl}_{3}\right)$ ठ 7.79-7.70 (m, 2H), $7.49(\mathrm{~s}, 1 \mathrm{H}), 7.45-7.39(\mathrm{~m}, 1 \mathrm{H}), 7.36-7.31$ $(\mathrm{m}, 1 \mathrm{H}), 7.12(\mathrm{~s}, 1 \mathrm{H}), 3.36(\mathrm{~s}, 3 \mathrm{H}), 3.26(\mathrm{~d}, J=18.1 \mathrm{~Hz}, 1 \mathrm{H}), 3.20(\mathrm{~d}, J=18.1 \mathrm{~Hz}, 1 \mathrm{H})$, 2.24-2.13 (m, 1H), 1.77-1.65 (m, 4H), 1.62-1.55 (m, 1H), $1.40(\mathrm{~s}, 3 \mathrm{H}), 1.25-1.03(\mathrm{~m}, 5 \mathrm{H})$; ${ }^{13} \mathrm{C}$ NMR $\left(101 \mathrm{MHz} \mathrm{CDCl}_{3}\right) \delta 210.0,180.1,142.3,135.1,133.8,130.2,127.8,127.1$, 126.1, 123.9, 120.5, 103.7, 50.3 48.2, 44.5, 28.1, 28.0, 26.6, 25.6, 25.5, 25.4, 25.0; HRMS: (ESI) calcd for $\mathrm{C}_{22} \mathrm{H}_{25} \mathrm{NNaO}_{2}{ }^{+}[\mathrm{M}+\mathrm{Na}]^{+} 358.1777$; found 358.1770.

3-(2-cyclohexyl-2-oxoethyl)-1,3-dimethyl-1,3-dihydro-2H-pyrrolo[2,3-b]pyridin-2-one (4la) 


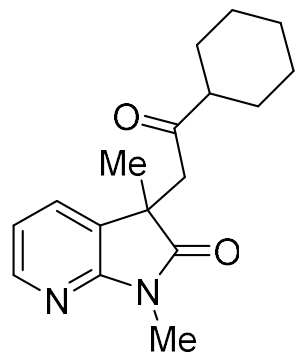

Chemical Formula: $\mathrm{C}_{17} \mathrm{H}_{22} \mathrm{~N}_{2} \mathrm{O}_{2}$

Exact Mass: 286.1681

4la was prepared according to general procedure 2.1 using $1 \mathbf{I}$ and $\mathbf{2 a}$ and was purified by silica gel column chromatography $(\mathrm{PE} / \mathrm{EtOAc}=10 / 1 \sim 2 / 1)$ to obtain 4la as colorless oil (28.0 mg, 49\% yield). ${ }^{1} \mathrm{H}$ NMR (400 MHz, $\mathrm{CDCl}_{3}$ ) $\delta 8.16$ (dd, $\left.J=5.3,1.6 \mathrm{~Hz}, 1 \mathrm{H}\right), 7.38$ (dd, $J=7.3,1.6 \mathrm{~Hz}, 1 \mathrm{H}), 6.88(\mathrm{dd}, J=7.2,5.3 \mathrm{~Hz}, 1 \mathrm{H}), 3.35(\mathrm{~s}, 3 \mathrm{H}), 3.16(\mathrm{~d}, J=18.1 \mathrm{~Hz}$, $1 \mathrm{H}), 3.10(\mathrm{~d}, J=18.1 \mathrm{~Hz}, 1 \mathrm{H}), 2.26-2.16(\mathrm{~m}, 1 \mathrm{H}), 1.77-1.67(\mathrm{~m}, 4 \mathrm{H}), 1.66-1.58(\mathrm{~m}, 1 \mathrm{H})$, 1.35 (s, 3H), 1.27-1.10 (m, 5H); ${ }^{13} \mathrm{C}$ NMR (101 MHz, $\left.\mathrm{CDCl}_{3}\right) \delta$ 210.0, 180.3, 157.3, 146.6, 129.2, 128.3, 117.8, 50.5, 47.38, 44.9, 28.3, 28.1, 25.7, 25.6, 25.5, 23.8; HRMS: (ESI) calcd for $\mathrm{C}_{17} \mathrm{H}_{22} \mathrm{~N}_{2} \mathrm{NaO}_{2}{ }^{+}[\mathrm{M}+\mathrm{Na}]^{+} 309.1574$; found 309.1580 .

1-(2-cyclohexyl-2-oxoethyl)-1,8-dimethyl-5,6-dihydro-4H-pyrrolo[3,2,1-ij]quinolin-2(1H)one (4ma)<smiles>Cc1cc2c3c(c1)C(C)(CC(=O)C1CCCCC1)C(=O)N3CCC2</smiles>

Chemical Formula: $\mathrm{C}_{21} \mathrm{H}_{27} \mathrm{NO}_{2}$

Exact Mass: 325.2042

4ma was prepared according to general procedure 2.1 using $1 \mathrm{~m}$ and $2 \mathrm{a}$ and was purified by silica gel column chromatography $(\mathrm{PE} / \mathrm{EtOAc}=10 / 1 \sim 5 / 1)$ to obtain $4 \mathrm{ma}$ as colorless oil (45.5 mg, 70\% yield). ${ }^{1} \mathrm{H}$ NMR (400 MHz, $\left.\mathrm{CDCl}_{3}\right) \delta$ 6.85-6.79 (m, 1H), 6.79-6.73 (m, $1 \mathrm{H}), 3.74(\mathrm{dd}, J=6.6,5.1 \mathrm{~Hz}, 2 \mathrm{H}), 3.10(\mathrm{~d}, J=17.8 \mathrm{~Hz}, 1 \mathrm{H}), 3.03(\mathrm{~d}, J=17.7 \mathrm{~Hz}, 1 \mathrm{H})$, 2.82-2.67 (m, 2H), $2.27(\mathrm{~s}, 3 \mathrm{H}), 2.24-2.15(\mathrm{~m}, 1 \mathrm{H}), 2.11-1.94(\mathrm{~m}, 2 \mathrm{H}), 1.76-1.67(\mathrm{~m}, 4 \mathrm{H})$, 1.64-1.56 (m, 1H), $1.33(\mathrm{~s}, 3 \mathrm{H}), 1.29-1.05(\mathrm{~m}, 5 \mathrm{H}) ;{ }^{13} \mathrm{C} \mathrm{NMR}\left(101 \mathrm{MHz}, \mathrm{CDCl}_{3}\right) \delta 210.2$, 179.2 , 137.0, 132.2, 131.0, 127.0, 120.5, 119.7, 50.5, 47.5, 46.4, 38.8, 28.2, 28.0, 25.7, 
25.6, 25.4, 24.5, 24.2, 21.3; HRMS: (ESI) calcd for $\left.\mathrm{C}_{21} \mathrm{H}_{27} \mathrm{NNaO}_{2}+\mathrm{M}+\mathrm{Na}\right]^{+} 348.1934$; found 348.1933 .

3-(2-cyclohexyl-2-oxoethyl)-3-isopropyl-1-methylindolin-2-one (4na)<smiles>CC(C)C1(CC(=O)C2CCCCC2)C(=O)N(C)c2ccccc21</smiles>

Chemical Formula: $\mathrm{C}_{20} \mathrm{H}_{27} \mathrm{NO}_{2}$

Exact Mass: 313.2042

4na was prepared according to general procedure 2.1 using $1 \mathbf{n}$ and $2 \mathbf{a}$ and was purified by silica gel column chromatography $(\mathrm{PE} / \mathrm{EtOAc}=10 / 1 \sim 5 / 1)$ to obtain 4 na as colorless oil (20.0 mg, 32\% yield). ' $\mathrm{H}$ NMR (400 MHz, $\left.\mathrm{CDCl}_{3}\right)$ ठ 7.26-7.21 (m, 1H), 7.06-7.02 (m, 1H), 6.99-6.94 (m, 1H), 6.85-6.81 (m, 1H), $3.28(\mathrm{~d}, J=17.7 \mathrm{~Hz}, 1 \mathrm{H}), 3.25(\mathrm{~s}, 3 \mathrm{H}), 3.07(\mathrm{~d}, \mathrm{~J}=$ $17.6 \mathrm{~Hz}, 1 \mathrm{H}), 2.23-2.13(\mathrm{~m}, 1 \mathrm{H}), 2.12-2.02(\mathrm{~m}, 1 \mathrm{H}), 1.71-1.64(\mathrm{~m}, 4 \mathrm{H}), 1.62-1.56(\mathrm{~m}, 1 \mathrm{H})$, 1.23-0.99 (m, 5H), $0.94(\mathrm{~d}, J=6.9 \mathrm{~Hz}, 3 \mathrm{H}), 0.68(\mathrm{~d}, J=6.8 \mathrm{~Hz}, 3 \mathrm{H}) ;{ }^{13} \mathrm{C}$ NMR $(101 \mathrm{MHz}$, $\left.\mathrm{CDCl}_{3}\right)$ $\delta 210.3,180.1,145.2,130.6,127.9,122.6,121.7,107.8,52.4,50.7,46.0,35.5$, 28.4, 28.1, 26.2, 25.8, 25.7, 25.5, 17.3, 16.8; HRMS: (ESI) calcd for $\mathrm{C}_{20} \mathrm{H}_{27} \mathrm{NNaO}_{2}{ }^{+}$ $[\mathrm{M}+\mathrm{Na}]^{+} 336.1934$; found 336.1936 .

3-(2-cyclohexyl-2-oxoethyl)-3-hexyl-1-methylindolin-2-one (4oa)

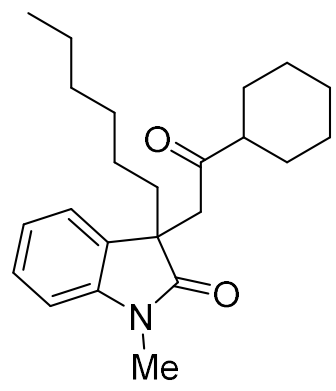

Chemical Formula: $\mathrm{C}_{23} \mathrm{H}_{33} \mathrm{NO}_{2}$

Exact Mass: 355.2511 
4oa was prepared according to general procedure 2.1 using 10 and $2 \mathrm{a}$ and was purified by silica gel column chromatography $(\mathrm{PE} / \mathrm{EtOAc}=15 / 1 \sim 10 / 1)$ to obtain 4 oa as colorless oil (36.2 mg, 51\% yield). ${ }^{1} \mathrm{H}$ NMR (400 MHz, $\left.\mathrm{CDCl}_{3}\right) \delta$ 7.26-7.21 (m, 1H), 7.08-7.04 (m, $1 \mathrm{H}), 7.01-6.95(\mathrm{~m}, 1 \mathrm{H}), 6.86-6.81(\mathrm{~m}, 1 \mathrm{H}), 3.26(\mathrm{~s}, 3 \mathrm{H}), 3.16(\mathrm{~d}, J=17.6 \mathrm{~Hz}, 1 \mathrm{H}), 3.05(\mathrm{~d}$, $J=17.7 \mathrm{~Hz}, 1 \mathrm{H}), 2.21-2.11(\mathrm{~m}, 1 \mathrm{H}), 1.84-1.64(\mathrm{~m}, 7 \mathrm{H}), 1.63-1.56(\mathrm{~m}, 1 \mathrm{H}), 1.24-1.00(\mathrm{~m}$, $12 \mathrm{H}), 0.80$ (t, $J=7.0 \mathrm{~Hz}, 3 \mathrm{H}) ;{ }^{13} \mathrm{C}$ NMR $\left(101 \mathrm{MHz}, \mathrm{CDCl}_{3}\right) \delta 210.0,180.0,144.6,132.1$, 127.7, 121.9, 121.7, 107.9, 50.5, 49.2, 47.5, 38.3, 31.4, 29.3, 28.2, 27.9, 26.2, 25.7, 25.6, 25.4, 23.2, 22.5, 14.0; HRMS: (ESI) calcd for $\mathrm{C}_{23} \mathrm{H}_{33} \mathrm{NNaO}_{2}{ }^{+}[\mathrm{M}+\mathrm{Na}]^{+}$378.2403; found 378.2404 .

3-benzyl-3-(2-cyclohexyl-2-oxoethyl)-1-methylindolin-2-one (4pa)

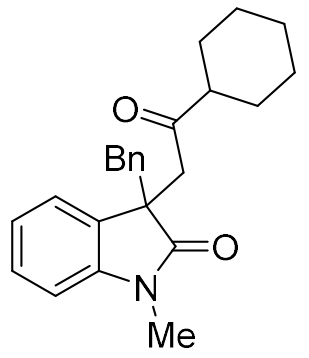

Chemical Formula: $\mathrm{C}_{24} \mathrm{H}_{27} \mathrm{NO}_{2}$

Exact Mass: 361.2042

4pa was prepared according to general procedure 2.1 using $1 \mathrm{p}$ and $2 \mathrm{a}$ and was purified by silica gel column chromatography $(\mathrm{PE} / \mathrm{EtOAc}=10 / 1 \sim 5 / 1)$ to obtain $4 \mathrm{pa}$ as colorless oil (48.4 mg, 67\% yield). The ${ }^{1} \mathrm{H}$ NMR data matched those reported in the literature. ${ }^{2}{ }^{1} \mathrm{H}$ NMR $\left(400 \mathrm{MHz} \mathrm{CDCl}_{3}\right)$ ठ 7.18-7.12 (m, 1H), 7.09-7.02 (m, 3H), 7.01-6.92 (m, 2H), 6.81-6.75 $(\mathrm{m}, 2 \mathrm{H}), 6.60-6.56(\mathrm{~m}, 1 \mathrm{H}), 3.23(\mathrm{~s}, 1 \mathrm{H}), 3.23(\mathrm{~s}, 1 \mathrm{H}), 3.23(\mathrm{~s}, 1 \mathrm{H}), 3.02(\mathrm{~d}, J=12.8 \mathrm{~Hz}$, $1 \mathrm{H}), 3.00(\mathrm{~s}, 3 \mathrm{H}), 2.96(\mathrm{~d}, J=12.7 \mathrm{~Hz}, 1 \mathrm{H}), 2.25-2.15(\mathrm{~m}, 1 \mathrm{H}), 1.78-1.65(\mathrm{~m}, 4 \mathrm{H}), 1.65-$ $1.57(\mathrm{~m}, 1 \mathrm{H}), 1.29-1.03(\mathrm{~m}, 5 \mathrm{H}) ;{ }^{13} \mathrm{C}$ NMR $\left(101 \mathrm{MHz}, \mathrm{CDCl}_{3}\right) \delta$ 209.8, 179.2, 135.0, 131.0, 130.1 , 128.0, 127.5, 126.7, 122.6, 121.7, 107.1, 50.8, 50.6, 46.8, 44.2, 28.3, 28.1, 26.1, $25.8,25.7,25.5$.

3-(2-cyclohexyl-2-oxoethyl)-3-(methoxymethyl)-1-methylindolin-2-one (4qa) 
<smiles>COCC1(CC(=O)C2CCCCC2)C(=O)N(C)c2ccccc21</smiles>

Chemical Formula: $\mathrm{C}_{19} \mathrm{H}_{25} \mathrm{NO}_{3}$

Exact Mass: 315.1834

4qa was prepared according to general procedure 2.1 using $1 \mathbf{q}$ and $\mathbf{2 a}$ and was purified by silica gel column chromatography $(\mathrm{PE} / \mathrm{EtOAc}=10 / 1 \sim 2 / 1)$ to obtain $\mathbf{4 q a}$ as colorless oil (22.1 mg, 35\% yield). ${ }^{1} \mathrm{H}$ NMR (400 MHz, $\left.\mathrm{CDCl}_{3}\right)$ ठ 7.28-7.23 (m, 1H), 7.21-7.18 (m, 1H), 7.01-6.96 (m, 1H), 6.87-6.83 (m, 1H), $3.62(\mathrm{~d}, J=8.8 \mathrm{~Hz}, 1 \mathrm{H}), 3.38(\mathrm{~d}, J=10.5 \mathrm{~Hz}, 1 \mathrm{H})$, 3.35 (d, J = 1.4 Hz, 1H), 3.26 (s, 3H), 3.25 (s, 3H), 3.05 (d, J=17.8 Hz, 1H), 2.25-2.15 (m, $1 \mathrm{H}), 1.75-1.66(\mathrm{~m}, 4 \mathrm{H}), 1.65-1.54(\mathrm{~m}, 1 \mathrm{H}), 1.28-1.03(\mathrm{~m}, 5 \mathrm{H}) ;{ }^{13} \mathrm{C} \mathrm{NMR}\left(101 \mathrm{MHz}, \mathrm{CDCl}_{3}\right)$ ठ 209.9, 177.9, 144.4, 130.8, 128.1, 122.9, 121.9, 108.0, 76.8, 59.52, 50.6, 50.4, 43.9, 28.2, 27.9, 26.4, 25.7, 25.5, 25.4; HRMS: (ESI) calcd for $\mathrm{C}_{19} \mathrm{H}_{25} \mathrm{NNaO}_{3}{ }^{+}[\mathrm{M}+\mathrm{Na}]^{+} 338.1727$; found 338.1722 .

1-benzyl-3-(2-cyclohexyl-2-oxoethyl)-3-methylindolin-2-one (4ra)<smiles>CC1(CC(=O)C2CCCCC2)C(=O)N(Cc2ccccc2)c2ccccc21</smiles>

Chemical Formula: $\mathrm{C}_{24} \mathrm{H}_{27} \mathrm{NO}_{2}$

Exact Mass: 361.2042

4ra was prepared according to general procedure 2.1 using $1 \mathbf{r}$ and $2 \mathbf{a}$ and was purified by silica gel column chromatography $(\mathrm{PE} / \mathrm{EtOAc}=10 / 1 \sim 5 / 1)$ to obtain 4ra as colorless oil (43.3 $\mathrm{mg}, 60 \%$ yield). The NMR data matched those reported in the literature. ${ }^{2}{ }^{1} \mathrm{H}$ NMR $\left(400 \mathrm{MHz} \mathrm{CDCl}_{3}\right)$ ठ 7.39-7.29 (m, 4H), 7.28-7.21 (m, 1H), 7.13-7.06 (m, 2H), 6.97-6.92 (m, 1H), 6.69 (dd, J = 8.3, 1.0 Hz, 1H), $5.08(\mathrm{~d}, J=15.9 \mathrm{~Hz}, 1 \mathrm{H}), 4.87(\mathrm{~d}, J=15.8 \mathrm{~Hz}, 1 \mathrm{H})$, $3.21(\mathrm{~d}, J=17.8 \mathrm{~Hz}, 1 \mathrm{H}), 3.14(\mathrm{~d}, J=17.9 \mathrm{~Hz}, 1 \mathrm{H}), 2.26-2.16(\mathrm{~m}, 1 \mathrm{H}), 1.79-1.69(\mathrm{~m}, 4 \mathrm{H})$, 
1.65-1.57 (m, 1H), $1.38(\mathrm{~s}, 3 \mathrm{H}), 1.30-1.09(\mathrm{~m}, 5 \mathrm{H}) ;{ }^{13} \mathrm{C} \mathrm{NMR}\left(101 \mathrm{MHz}, \mathrm{CDCl}_{3}\right) \delta 209.8$, $180.6,142.8,136.2,133.7,128.6,127.6,127.3,127.2,122.1,121.5,109.2,50.4,47.5$, $45.1,43.9,28.2,28.0,25.7,25.56,25.4,25.1$.

1,3-dimethyl-3-(2-oxo-6-phenylhexyl)indolin-2-one (4ab)

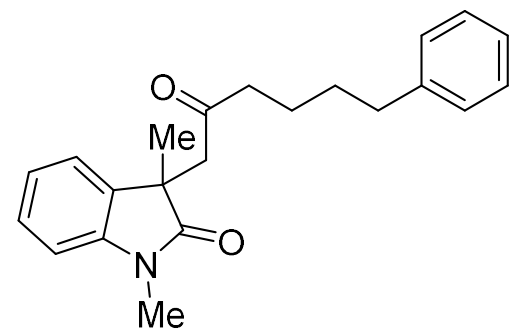

Chemical Formula: $\mathrm{C}_{22} \mathrm{H}_{25} \mathrm{NO}_{2}$

Exact Mass: 335.1885

4ab was prepared according to general procedure 2.1 using $\mathbf{1 a}$ and $\mathbf{2} \mathbf{b}$ and was purified by silica gel column chromatography $(\mathrm{PE} / \mathrm{EtOAc}=10 / 1 \sim 3 / 1)$ to obtain $4 \mathrm{ab}$ as colorless oil (33.5 mg, 50\% yield). ${ }^{1} \mathrm{H}$ NMR (400 MHz, $\left.\mathrm{CDCl}_{3}\right)$ ठ 7.27-7.21 (m, 3H), 7.18-7.12 (m, 1H), 7.11-7.06 (m, 3H), 7.01-6.95 (m, 1H), 6.87-6.82 (m, 1H), $3.26(\mathrm{~s}, 3 \mathrm{H}), 3.07(\mathrm{~d}, J=17.6$ $\mathrm{Hz}, 1 \mathrm{H}), 3.02(\mathrm{~d}, J=17.6 \mathrm{~Hz}, 1 \mathrm{H}), 2.55-2.48(\mathrm{~m}, 2 \mathrm{H}), 2.33-2.19(\mathrm{~m}, 2 \mathrm{H}), 1.49-1.42(\mathrm{~m}$, 4H), 1.32 (s, 3H); ${ }^{13} \mathrm{C}$ NMR (101 MHz, $\left.\mathrm{CDCl}_{3}\right) \delta$ 206.8, 180.3, 143.7, 142.1, 133.5, 128.3, 128.2, 127.8, 125.7, 122.1, 121.7, 108.1, 49.6, 45.1, 42.6, 35.6, 30.7, 26.3, 24.4, 23.0; HRMS: (ESI) calcd for $\mathrm{C}_{22} \mathrm{H}_{25} \mathrm{NNaO}_{2}{ }^{+}[\mathrm{M}+\mathrm{Na}]^{+} 358.1777$; found 358.1777.

7-(1,3-dimethyl-2-oxoindolin-3-yl)-6-oxoheptanenitrile (4ac)

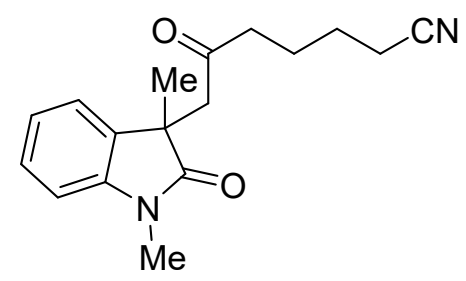

Chemical Formula: $\mathrm{C}_{17} \mathrm{H}_{20} \mathrm{~N}_{2} \mathrm{O}_{2}$

Exact Mass: 284.1525

4ac was prepared according to general procedure 2.1 using $1 \mathbf{a}$ and $\mathbf{2 c}$ and was purified by silica gel column chromatography $(\mathrm{PE} / \mathrm{EtOAc}=10 / 1 \sim 1 / 2)$ to obtain 4 ac as colorless oil 
(23.9 mg, 42\% yield). ${ }^{1} \mathrm{H}$ NMR (400 MHz, $\left.\mathrm{CDCl}_{3}\right)$ ठ 7.30-7.24 (m, 1H), 7.15-7.10 (m, 1H), 7.04-6.99 (m, 1H), 6.89-6.85 (m, 1H), $3.27(\mathrm{~s}, 3 \mathrm{H}), 3.09(\mathrm{~d}, J=17.4 \mathrm{~Hz}, 1 \mathrm{H}), 3.04(\mathrm{~d}, J=$ $17.4 \mathrm{~Hz}, 1 \mathrm{H}), 2.40-2.27(\mathrm{~m}, 2 \mathrm{H}), 2.24-2.19(\mathrm{~m}, 2 \mathrm{H}), 1.57-1.42(\mathrm{~m}, 4 \mathrm{H}), 1.33(\mathrm{~s}, 3 \mathrm{H}) ;{ }^{13} \mathrm{C}$ NMR $\left(101 \mathrm{MHz}, \mathrm{CDCl}_{3}\right) \delta 205.8,180.2,143.6,133.2,128.0,122.2,121.6,119.3,108.2$, 49.6, 45.2, 41.5, 26.3, 24.5, 24.4, 22.2, 16.9; HRMS: (ESI) calcd for $\mathrm{C}_{17} \mathrm{H}_{20} \mathrm{~N}_{2} \mathrm{NaO}_{2}{ }^{+}[\mathrm{M}+\mathrm{Na}]^{+}$307.1417; found 307.1415.

methyl 7-(1,3-dimethyl-2-oxoindolin-3-yl)-6-oxoheptanoate (4ad)

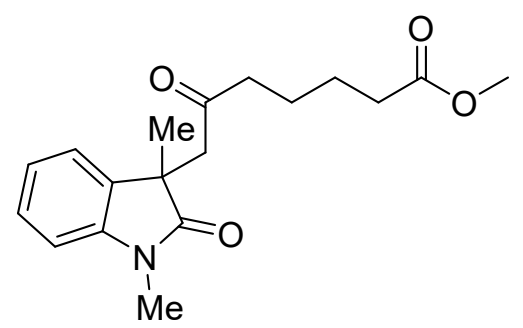

Chemical Formula: $\mathrm{C}_{18} \mathrm{H}_{23} \mathrm{NO}_{4}$

Exact Mass: 317.1627

4ad was prepared according to general procedure 2.1 using $\mathbf{1 a}$ and $\mathbf{2 d}$ and was purified by silica gel column chromatography $(\mathrm{PE} / \mathrm{EtOAc}=10 / 1 \sim 1 / 1)$ to obtain 4ad as colorless oil (38.1 mg, 60\% yield). ${ }^{1} \mathrm{H}$ NMR (400 MHz, $\left.\mathrm{CDCl}_{3}\right) \delta$ 7.28-7.22 (m, 1H), 7.12 (dd, J = 7.5, $1.6 \mathrm{~Hz}, 1 \mathrm{H}), 7.03-6.97(\mathrm{~m}, 1 \mathrm{H}), 6.88-6.84(\mathrm{~m}, 1 \mathrm{H}), 3.63(\mathrm{~s}, 3 \mathrm{H}), 3.26(\mathrm{~s}, 3 \mathrm{H}), 3.09(\mathrm{~d}, J=$ $16.0 \mathrm{~Hz}, 1 \mathrm{H}), 3.04(\mathrm{~d}, J=16.0 \mathrm{~Hz}, 1 \mathrm{H}), 2.36-2.18(\mathrm{~m}, 4 \mathrm{H}), 1.51-1.38(\mathrm{~m}, 4 \mathrm{H}), 1.33(\mathrm{~s}, 3 \mathrm{H})$; ${ }^{13} \mathrm{C}$ NMR $\left(101 \mathrm{MHz}, \mathrm{CDCl}_{3}\right) \delta 206.4,180.3,173.7,143.6,133.4,127.8,122.2,121.7$, 108.1, 51.4, 49.6, 45.1, 42.2, 33.6, 26.3, 24.4, 24.1, 22.7; HRMS: (ESI) calcd for $\mathrm{C}_{18} \mathrm{H}_{23} \mathrm{NNaO}_{4}{ }^{+}[\mathrm{M}+\mathrm{Na}]^{+} 340.1519$; found 340.1511 .

1,3-dimethyl-3-(2-oxo-5-phenoxypentyl)indolin-2-one (4ae) 


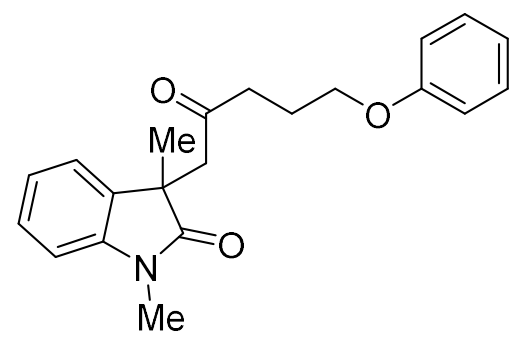

Chemical Formula: $\mathrm{C}_{21} \mathrm{H}_{23} \mathrm{NO}_{3}$

Exact Mass: 337.1678

4ae was prepared according to general procedure 2.1 using $N$-(2-iodophenyl)- $N$ methylmethacrylamide and $2 \mathrm{e}$ and was purified by silica gel column chromatography $(\mathrm{PE} / \mathrm{EtOAc}=10 / 1 \sim 2 / 1)$ to obtain 4ae as colorless oil (43.2 mg, 64\% yield). ${ }^{1} \mathrm{H}$ NMR (400 $\left.\mathrm{MHz}, \mathrm{CDCl}_{3}\right) \delta$ 7.27-7.21 (m, 3H), 7.12-7.08 (m, 1H), 6.99-6.94 (m, 1H), 6.94-6.89 (m, 1H), 6.87-6.83 (m, 1H), 6.82-6.77 (m, 2H), 3.84-3.71 (m, 2H), $3.26(\mathrm{~s}, 3 \mathrm{H}), 3.12(\mathrm{~d}, J=17.6$ $\mathrm{Hz}, 1 \mathrm{H}), 3.07(\mathrm{~d}, J=17.6 \mathrm{~Hz}, 1 \mathrm{H}), 2.56-2.39(\mathrm{~m}, 2 \mathrm{H}), 1.91-1.82(\mathrm{~m}, 2 \mathrm{H}), 1.33(\mathrm{~s}, 3 \mathrm{H}) ;{ }^{13} \mathrm{C}$ NMR (101 MHz, $\left.\mathrm{CDCl}_{3}\right) \delta 206.3,180.2,158.7,143.6,133.3,129.3,127.9,122.2,121.7$, 120.6, 114.4, 108.1, 66.4, 49.7, 45.2, 39.0, 26.3, 24.4, 23.0; HRMS: (ESI) calcd for $\mathrm{C}_{21} \mathrm{H}_{23} \mathrm{NNaO}_{3}{ }^{+}[\mathrm{M}+\mathrm{Na}]^{+} 360.1570$; found 360.1570 .

2-(4-(1,3-dimethyl-2-oxoindolin-3-yl)-3-oxobutyl)isoindoline-1,3-dione (4af)

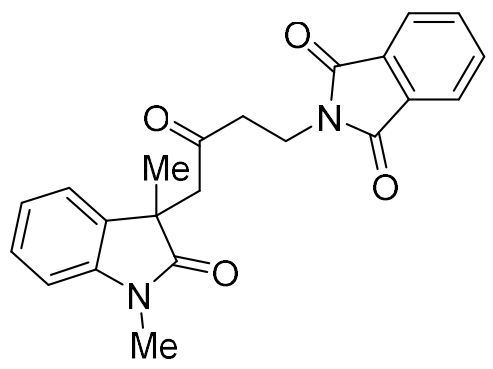

Chemical Formula: $\mathrm{C}_{22} \mathrm{H}_{20} \mathrm{~N}_{2} \mathrm{O}_{4}$

Exact Mass: 376.1423

4af was prepared according to general procedure 2.1 using $\mathbf{1 a}$ and $\mathbf{2 f}$ and was purified by silica gel column chromatography $(\mathrm{PE} / \mathrm{EtOAc}=10 / 1 \sim 1 / 2)$ to obtain 4af as colorless oil (36.1 mg, 48\% yield). ${ }^{1} \mathrm{H}$ NMR (400 MHz, $\mathrm{CDCl}_{3}$ ) $\delta 7.80$ (dd, $\left.J=5.5,3.0 \mathrm{~Hz}, 2 \mathrm{H}\right), 7.70$ (dd, $J=5.4,3.1 \mathrm{~Hz}, 2 \mathrm{H}), 7.25-7.20(\mathrm{~m}, 1 \mathrm{H}), 7.13(\mathrm{dd}, J=7.3,1.3 \mathrm{~Hz}, 1 \mathrm{H}), 7.01-6.95(\mathrm{~m}$, $1 \mathrm{H}), 6.87-6.83(\mathrm{~m}, 1 \mathrm{H}), 3.82-3.71(\mathrm{~m}, 2 \mathrm{H}), 3.26(\mathrm{~s}, 3 \mathrm{H}), 3.13(\mathrm{~d}, J=28.7 \mathrm{~Hz}, 1 \mathrm{H}), 3.05(\mathrm{~d}$, $J=32.5 \mathrm{~Hz}, 1 \mathrm{H}), 2.78-2.63(\mathrm{~m}, 2 \mathrm{H}), 1.33(\mathrm{~s}, 3 \mathrm{H}) ;{ }^{13} \mathrm{C} \mathrm{NMR}\left(101 \mathrm{MHz}, \mathrm{CDCl}_{3}\right) \delta 203.9$, $180.0,167.9,143.5,133.9,133.1,131.9,127.9,123.2,122.3,121.9,108.2,49.5,45.1$, 
40.6, 32.5, 26.4, 24.4; HRMS: (ESI) calcd for $\mathrm{C}_{22} \mathrm{H}_{20} \mathrm{~N}_{2} \mathrm{NaO}_{4}{ }^{+}[\mathrm{M}+\mathrm{Na}]^{+}$399.1315; found 399.1312 .

3-(6-chloro-2-oxohexyl)-1,3-dimethylindolin-2-one (4ag)

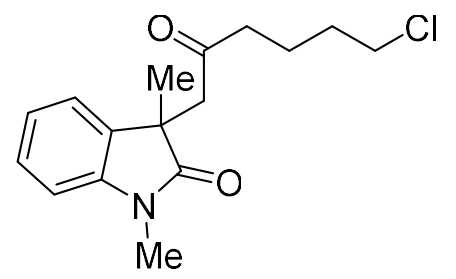

Chemical Formula: $\mathrm{C}_{16} \mathrm{H}_{20} \mathrm{CINO}_{2}$

Exact Mass: 293.1183

4ag was prepared according to general procedure 2.1 using $\mathbf{1 a}$ and $\mathbf{2} \mathbf{g}$ and was purified by silica gel column chromatography $(\mathrm{PE} / \mathrm{EtOAc}=10 / 1 \sim 2 / 1)$ to obtain 4 ag as colorless oil (33.4 mg, 57\% yield). ${ }^{1} \mathrm{H}$ NMR (400 MHz, $\left.\mathrm{CDCl}_{3}\right)$ ठ 7.29-7.22 (m, 1H), 7.14-7.10 (m, 1H), 7.04-6.98 (m, 1H), 6.89-6.84 (m, 1H), $3.42(\mathrm{t}, J=6.1 \mathrm{~Hz}, 2 \mathrm{H}), 3.27(\mathrm{~s}, 3 \mathrm{H}), 3.09(\mathrm{~d}, J=$ $20.0 \mathrm{~Hz}, 1 \mathrm{H}), 3.05(\mathrm{~d}, J=20.0 \mathrm{~Hz}, 1 \mathrm{H}), 2.38-2.22(\mathrm{~m}, 2 \mathrm{H}), 1.64-1.50(\mathrm{~m}, 4 \mathrm{H}), 1.33(\mathrm{~s}, 3 \mathrm{H})$; ${ }^{13} \mathrm{C} \mathrm{NMR}\left(101 \mathrm{MHz}, \mathrm{CDCl}_{3}\right) \delta 206.2,143.6,133.3,127.9,122.2,121.6,108.2,49.6,45.2$, 44.5, 41.7, 31.6, 26.4, 24.5, 20.6; HRMS: (ESI) calcd for $\mathrm{C}_{16} \mathrm{H}_{20} \mathrm{CINNaO}_{2}{ }^{+}[\mathrm{M}+\mathrm{Na}]^{+}$ 316.1075; found 316.1071 .

1,3-dimethyl-3-(2-oxo-2-(tetrahydro-2H-pyran-4-yl)ethyl)indolin-2-one (4ah)<smiles>[M]C1(CC(=O)C2CCOCC2)C(=O)N(C)c2ccccc21</smiles>

Chemical Formula: $\mathrm{C}_{17} \mathrm{H}_{21} \mathrm{NO}_{3}$

Exact Mass: 287.1521

4ah was prepared according to general procedure 2.1 using $\mathbf{1 a}$ and $\mathbf{2 h}$ and was purified by silica gel column chromatography $(\mathrm{PE} / \mathrm{EtOAc}=10 / 1 \sim 2 / 1)$ to obtain 4ah as colorless oil (32.1 mg, 56\% yield). ${ }^{1} \mathrm{H}$ NMR (400 MHz, $\left.\mathrm{CDCl}_{3}\right) \delta$ 7.31-7.20 (m, 1H), 7.10 (dd, J = 7.3, $1.3 \mathrm{~Hz}, 1 \mathrm{H}), 7.05-6.95(\mathrm{~m}, 1 \mathrm{H}), 6.87$ (dd, $J=7.8,0.9 \mathrm{~Hz}, 1 \mathrm{H}), 3.97-3.85(\mathrm{~m}, 2 \mathrm{H}), 3.34$ (td, 
$J=11.5,2.7 \mathrm{~Hz}, 3 \mathrm{H}), 3.27(\mathrm{~s}, 3 \mathrm{H}), 3.15(\mathrm{~d}, J=17.8 \mathrm{~Hz}, 2 \mathrm{H}), 3.10(\mathrm{~d}, J=17.8 \mathrm{~Hz}, 2 \mathrm{H})$, 2.47-2.32 (m, 1H), 1.70-1.42 (m, 5H), 1.34 (s, 3H); $\left.{ }^{13} \mathrm{C} \mathrm{NMR} \mathrm{(101} \mathrm{MHz,} \mathrm{CDCl}_{3}\right) \delta 207.9$, 180.3, 143.7, 133.4, 127.9, 122.2, 121.5, 108.2, 67.0, 67.0, 47.4, 47.3, 45.0, 27.8, 27.6, 26.4, 24.5; HRMS: (ESI) calcd for $\mathrm{C}_{17} \mathrm{H}_{21} \mathrm{NNaO}_{3}{ }^{+}[\mathrm{M}+\mathrm{Na}]^{+} 310.1414$; found 310.1412.

3-(4-(1H-indol-1-yl)-2-oxobutyl)-1,3-dimethylindolin-2-one (4ai)

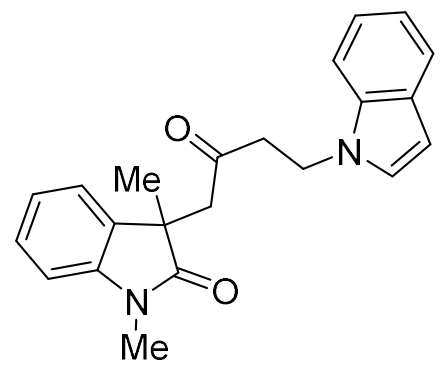

Chemical Formula: $\mathrm{C}_{22} \mathrm{H}_{22} \mathrm{~N}_{2} \mathrm{O}_{2}$

Exact Mass: 346.1681

4ai was prepared according to general procedure 2.1 using $N$-(2-iodophenyl)- $N$ methylmethacrylamide and $\mathbf{2} \mathbf{i}$ and was purified by silica gel column chromatography $(\mathrm{PE} / \mathrm{EtOAc}=10 / 1 \sim 2 / 1)$ to obtain 4ai as colorless oil $\left(47.8 \mathrm{mg}, 69 \%\right.$ yield). ${ }^{1} \mathrm{H}$ NMR (400 $\left.\mathrm{MHz}, \mathrm{CDCl}_{3}\right) \delta$ 7.61-7.57 (m, 1H), 7.28-7.22 (m, 1H), 7.19-7.13 (m, 2H), 7.11-7.06 (m, 1H), 7.02-6.95 (m, 2H), $6.87(\mathrm{~d}, J=3.2 \mathrm{~Hz}, 1 \mathrm{H}), 6.86-6.82(\mathrm{~m}, 1 \mathrm{H}), 6.40(\mathrm{~d}, J=3.2 \mathrm{~Hz}, 1 \mathrm{H})$, $4.21(\mathrm{t}, J=6.9 \mathrm{~Hz}, 2 \mathrm{H}), 3.23(\mathrm{~s}, 3 \mathrm{H}), 3.03(\mathrm{~d}, J=17.4 \mathrm{~Hz}, 1 \mathrm{H}), 2.90(\mathrm{~d}, J=17.4 \mathrm{~Hz}, 1 \mathrm{H})$, 2.81-2.65 (m, 2H), 1.29 (s, 3H); ${ }^{13} \mathrm{C}$ NMR (101 MHz, $\left.\mathrm{CDCl}_{3}\right) \delta$ 204.7, 178.0, 143.4, 135.4, 133.0, 128.6, 128.0, 127.8, 122.3, 121.8, 121.5, 121.0, 119.4, 109.0, 108.2, 101.4, 50.0, 45.2, 42.8, 40.3, 26.3, 24.4; HRMS: (ESI) calcd for $\mathrm{C}_{22} \mathrm{H}_{22} \mathrm{~N}_{2} \mathrm{NaO}_{2}{ }^{+}[\mathrm{M}+\mathrm{Na}]^{+} 369.1556$; found 369.1573 .

3-(2-((3S,8S,9S, 10R,13R,14S,17R)-10,13-dimethyl-17-((R)-6-methylheptan-2-yl)2,3,4,7,8,9,10,11,12,13,14,15,16,17-tetradecahydro-1H-cyclopenta[a]phenanthren-3-yl)2-oxoethyl)-1,3-dimethylindolin-2-one (4aj) 


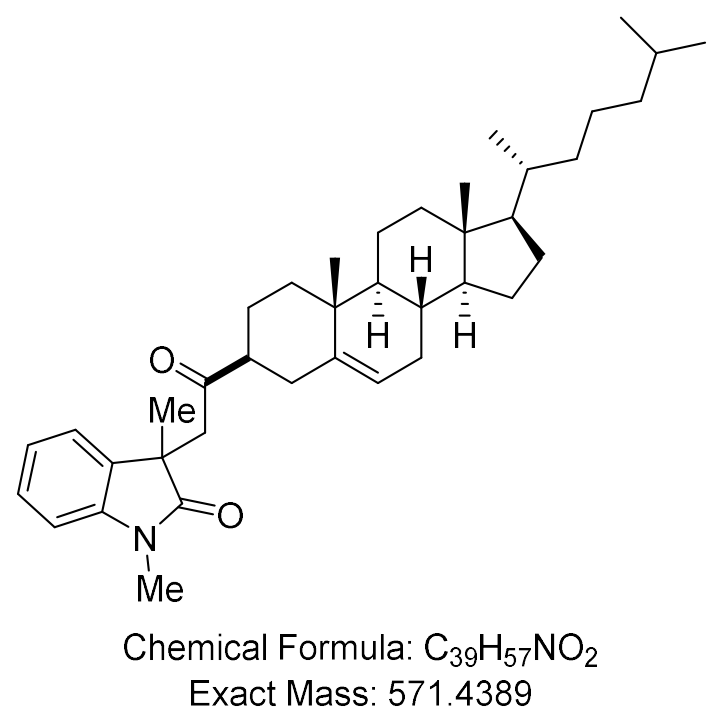

4aj was prepared according to general procedure 2.1 using $\mathrm{N}$-(2-iodophenyl)- $\mathrm{N}$ methylmethacrylamide and $\mathbf{2} \mathbf{j}$ and was purified by silica gel column chromatography $(\mathrm{PE} / \mathrm{EtOAc}=10 / 1 \sim 3 / 1)$ to obtain 4 aj as colorless oil $(67.4 \mathrm{mg}, 59 \%$ yield, d.r. $3 / 1) .{ }^{1} \mathrm{H}$ NMR $\left(400 \mathrm{MHz}, \mathrm{CDCl}_{3}\right)$ ठ 7.29-7.19 (m, 1H), 7.15-7.07 (m, 1H), 7.05-6.91 (m, 1H), 6.90-6.81 $(\mathrm{m}, 1 \mathrm{H}), 5.37-5.24(\mathrm{~m}, 1 \mathrm{H}), 3.27(\mathrm{~s}, 3 \mathrm{H}), 3.21-2.98(\mathrm{~m}, 2 \mathrm{H}), 2.60-2.16(\mathrm{~m}, 1 \mathrm{H}), 2.15-1.76$ $(\mathrm{m}, 5 \mathrm{H}), 1.73-1.21(\mathrm{~m}, 16 \mathrm{H}), 1.18-0.78(\mathrm{~m}, 24 \mathrm{H}), 0.65(\mathrm{~s}, 3 \mathrm{H}) ;{ }^{13} \mathrm{C} \mathrm{NMR}\left(101 \mathrm{MHz}, \mathrm{CDCl}_{3}\right)$ б 209.6, 208.8, 180.5, 180.4, 143.8, 141.1, 141.0, 139.4, 133.6, 133.6, 127.8, 127.7, 122.1, $121.9,121.9,121.7,121.5,120.9,120.9,108.1,108.1,56.7,56.5,56.1,56.0,51.8,51.6$, $50.2,49.7,48.5,48.2,48.1,47.4,45.3,45.0,42.2,42.2$, 39.7, 39.6, 39.5, 38.8, 38.7, 36.9, $36.9,36.9,36.2,35.8,35.7,35.3,34.1,33.9,32.9,31.9,31.7,31.7,31.6,28.2,28.0,26.4$, 24.6, 24.5, 24.5, 24.3, 24.2, 23.8, 23.8, 22.8, 22.5, 22.4, 20.8, 20.6, 19.3, 19.2, 18.7, 11.8; HRMS: (ESI) calcd for $\mathrm{C}_{39} \mathrm{H}_{57} \mathrm{NNaO}_{2}{ }^{+}[\mathrm{M}+\mathrm{Na}]^{+} 594.4281$; found 594.4277 .

1,3-dimethyl-3-(2-oxo-3-phenylpropyl)indolin-2-one (4ak)<smiles>[M]C1(CC(=O)Cc2ccccc2)C(=O)N(C)c2ccccc21</smiles>

Chemical Formula: $\mathrm{C}_{19} \mathrm{H}_{19} \mathrm{NO}_{2}$

Exact Mass: 293.1416 
4ak was prepared according to general procedure 2.2 using $\mathbf{1 a}$ and $\mathbf{2 k}$ and was purified by silica gel column chromatography $(\mathrm{PE} / \mathrm{EtOAc}=10 / 1 \sim 2 / 1)$ to obtain 4ak as colorless oil (40.4 mg, 69\% yield). ${ }^{1} \mathrm{H}$ NMR (400 MHz, $\left.\mathrm{CDCl}_{3}\right)$ ठ 7.30-7.19 (m, 4H), 7.04-6.99 (m, 2H), 6.96-6.92 (m, 2H), 6.85-6.81 (m, 1H), $3.52(\mathrm{~d}, J=1.2 \mathrm{~Hz}, 2 \mathrm{H}), 3.24(\mathrm{~s}, 3 \mathrm{H}), 3.11(\mathrm{~d}, J=$ $20.1 \mathrm{~Hz}, 1 \mathrm{H}), 3.06(\mathrm{~d}, J=20.1 \mathrm{~Hz}, 1 \mathrm{H}), 1.28(\mathrm{~s}, 3 \mathrm{H}) ;{ }^{13} \mathrm{C} \mathrm{NMR}\left(101 \mathrm{MHz}, \mathrm{CDCl}_{3}\right) \delta 204.3$, 180.4 , 143.8, 133.6, 133.4, 129.5, 128.8, 128.0, 127.2, 122.3, 121.9, 108.3, 50.2, 48.8, 45.3, 26.5, 24.5; HRMS: (ESI) calcd for $\mathrm{C}_{19} \mathrm{H}_{19} \mathrm{NNaO}_{2}{ }^{+}[\mathrm{M}+\mathrm{Na}]^{+} 316.1308$; found 316.1307.

1,3-dimethyl-3-(2-oxo-3-(4-(trifluoromethyl)phenyl)propyl)indolin-2-one (4al)<smiles>CN1C(=O)C(C)(CC(=O)Cc2ccc(C(F)(F)F)cc2)c2ccccc21</smiles>

Chemical Formula: $\mathrm{C}_{20} \mathrm{H}_{18} \mathrm{~F}_{3} \mathrm{NO}_{2}$

Exact Mass: 361.1290

4al was prepared according to general procedure 2.2 using $N$-(2-iodophenyl)- $N$ methylmethacrylamide and $\mathbf{2}$ land was purified by silica gel column chromatography $(\mathrm{PE} / \mathrm{EtOAc}=10 / 1 \sim 2 / 1)$ to obtain 4 al as colorless oil $\left(40.4 \mathrm{mg}, 56 \%\right.$ yield). ${ }^{1} \mathrm{H}$ NMR (400 $\left.\mathrm{MHz}, \mathrm{CDCl}_{3}\right) \delta$ 7.56-7.45 (m, 2H), 7.27-7.21 (m, 1H), 7.15-7.09 (m, 2H), 7.01-6.93 (m, 2H), 6.87-6.82 (m, 1H), $3.62(\mathrm{~s}, 2 \mathrm{H}), 3.25$ (s, 3H), 3.15 (d, J = $17.6 \mathrm{~Hz}, 1 \mathrm{H}), 3.10$ (d, J = 17.6 $\mathrm{Hz}, 1 \mathrm{H}), 1.32$ (s, 3H); ${ }^{13} \mathrm{C}$ NMR (101 MHz, $\left.\mathrm{CDCl}_{3}\right) \delta$ 203.1, 180.0, 143.6, 137.3, 133.0, 129.8, 129.4 (q $J=32.5 \mathrm{~Hz}$ ), 128.0, 124.1 (q, $J=273.7 \mathrm{~Hz}), 125.5$ (q, $J=3.8 \mathrm{~Hz}), 122.2$, 121.7, 108.3, 49.5, 49.1, 45.2, 26.4, 24.4; ${ }^{19} \mathrm{~F}$ NMR (376 MHz, $\mathrm{CDCl}_{3}$ ) $\delta-62.48$; HRMS: (ESI) calcd for $\mathrm{C}_{20} \mathrm{H}_{18} \mathrm{~F}_{3} \mathrm{NNaO}_{2}{ }^{+}[\mathrm{M}+\mathrm{Na}]^{+} 384.1182$; found 384.1179.

3-(3-(4-fluorophenyl)-2-oxopropyl)-1,3-dimethylindolin-2-one (4am) 


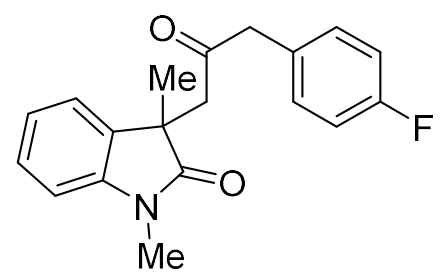

Chemical Formula: $\mathrm{C}_{19} \mathrm{H}_{18} \mathrm{FNO}_{2}$

Exact Mass: 311.1322

4am was prepared according to general procedure 2.2 using $\mathrm{N}$-(2-iodophenyl)- $\mathrm{N}$ methylmethacrylamide and $\mathbf{2 m}$ and was purified by silica gel column chromatography (PE/EtOAc $=10 / 1 \sim 2 / 1)$ to obtain 4 am as colorless oil (38.6 mg, 62\% yield). ${ }^{1} \mathrm{H}$ NMR (400 $\left.\mathrm{MHz}, \mathrm{CDCl}_{3}\right)$ ठ 7.26-7.21 (m, 1H), 7.01-6.91 (m, 6H), 6.86-6.82 (m, 1H), $3.51(\mathrm{~s}, 2 \mathrm{H}), 3.25$ (s, 3H), 3.10 (s, 2H), 1.30 (s, 3H); $\left.{ }^{13} \mathrm{C} \mathrm{NMR} \mathrm{(101} \mathrm{MHz,} \mathrm{CDCl}_{3}\right) \delta$ 204.0, 180.2, 162.1 (d, J $=245.5 \mathrm{~Hz}$ ), 143.8, 133.3, $131.0(\mathrm{~d}, J=8.1 \mathrm{~Hz}), 129.3(\mathrm{~d}, J=3.3 \mathrm{~Hz}), 128.0,122.3,121.8$, 115.6 (d, $J=21.4 \mathrm{~Hz}), 108.3,49.1,48.9,45.3,26.5,24.5$; ${ }^{19} \mathrm{~F} \mathrm{NMR}\left(376 \mathrm{MHz}, \mathrm{CDCl}_{3}\right) \delta$ 115.52; HRMS: (ESI) calcd for $\mathrm{C}_{19} \mathrm{H}_{18} \mathrm{FNNaO}_{2}{ }^{+}[\mathrm{M}+\mathrm{Na}]^{+} 334.1214$; found 334.1204.

3-(3-(4-chlorophenyl)-2-oxopropyl)-1,3-dimethylindolin-2-one (4an)<smiles>CN1C(=O)C(C)(CC(=O)Cc2ccc(Cl)cc2)c2ccccc21</smiles>

Chemical Formula: $\mathrm{C}_{19} \mathrm{H}_{18} \mathrm{ClNO}_{2}$

Exact Mass: 327.1026

4an was prepared according to general procedure 2.2 using $\mathrm{N}$-(2-iodophenyl)- $\mathrm{N}$ methylmethacrylamide and $\mathbf{2 n}$ and was purified by silica gel column chromatography $(\mathrm{PE} / \mathrm{EtOAc}=10 / 1 \sim 2 / 1)$ to obtain 4 an as colorless oil $\left(44.5 \mathrm{mg}, 68 \%\right.$ yield). ${ }^{1} \mathrm{H}$ NMR (400 $\left.\mathrm{MHz}, \mathrm{CDCl}_{3}\right)$ ठ 7.26-7.21 (m, 3H), 6.99-6.92 (m, 4H), 6.86-6.82 (m, 1H), $3.51(\mathrm{~s}, 2 \mathrm{H}), 3.24$ (s, 3H), 3.09 (s, 2H), 1.30 (s, 3H); ${ }^{13} \mathrm{C} \mathrm{NMR} \mathrm{(101} \mathrm{MHz,} \mathrm{CDCl} 3$ ) ठ 203.6, 180.1, 143.6, 133.1, 133.0, 131.8, 130.7, 128.8, 127.9, 122.2, 121.7, 108.2, 49.1 48.9, 45.2, 26.4, 24.4; HRMS: (ESI) calcd for $\mathrm{C}_{19} \mathrm{H}_{18} \mathrm{CINNaO}_{2}{ }^{+}[\mathrm{M}+\mathrm{Na}]^{+} 350.0918$; found 350.0907 .

3-(3-(3-methoxyphenyl)-2-oxopropyl)-1,3-dimethylindolin-2-one (4ao) 


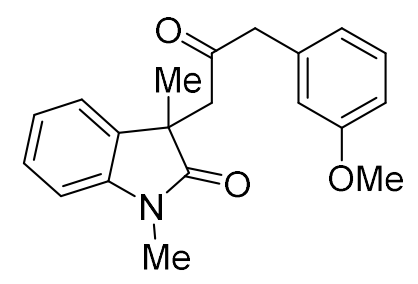

Chemical Formula: $\mathrm{C}_{20} \mathrm{H}_{21} \mathrm{NO}_{3}$

Exact Mass: 323.1521

4ao was prepared according to general procedure 2.2 using $1 \mathrm{a}$ and $\mathbf{2 0}$ and was purified by silica gel column chromatography (PE/EtOAc $=10 / 1 \sim 2 / 1)$ to obtain 4 ao as colorless oil (40.7 mg, 63\% yield). ${ }^{1} \mathrm{H} \mathrm{NMR}\left(400 \mathrm{MHz}, \mathrm{CDCl}_{3}\right) \delta$ 7.25-7.17 (m, 2H), 6.96-6.88 (m, 2H), 6.86-6.81 (m, 1H), 6.81-6.76 (m, 1H), 6.66-6.60 (m, 1H), $6.52(\mathrm{t}, J=2.1 \mathrm{~Hz}, 1 \mathrm{H}), 3.73(\mathrm{~s}$, $3 \mathrm{H}), 3.51(\mathrm{~d}, J=15.2 \mathrm{~Hz} 1 \mathrm{H}), 3.47(\mathrm{~d}, J=15.2 \mathrm{~Hz}, 1 \mathrm{H}), 3.25(\mathrm{~s}, 3 \mathrm{H}), 3.12(\mathrm{~d}, J=17.7 \mathrm{~Hz}$, $1 \mathrm{H}), 3.06(\mathrm{~d}, J=17.9 \mathrm{~Hz}, 1 \mathrm{H}), 1.28(\mathrm{~s}, 3 \mathrm{H}) ;{ }^{13} \mathrm{C} \mathrm{NMR}\left(101 \mathrm{MHz}, \mathrm{CDCl}_{3}\right) \delta 204.1,180.2$, 159.7, 143.7, 134.9, 133.2, 129.7, 127.8, 122.1, 121.7, 114.7, 112.8, 108.1, 55.1, 50.1, 48.5, 45.1, 26.3, 24.3; HRMS: (ESI) calcd for $\mathrm{C}_{20} \mathrm{H}_{21} \mathrm{NNaO}_{3}{ }^{+}[\mathrm{M}+\mathrm{Na}]^{+} 346.1414$; found 346.1414 . 


\section{Copies of the ${ }^{1} \mathrm{H},{ }^{19} \mathrm{~F}$ and ${ }^{13} \mathrm{C}$ NMR spectra}

4aa

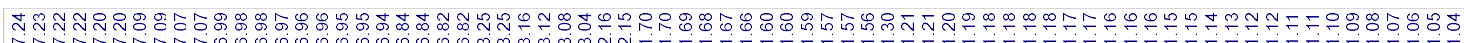
年
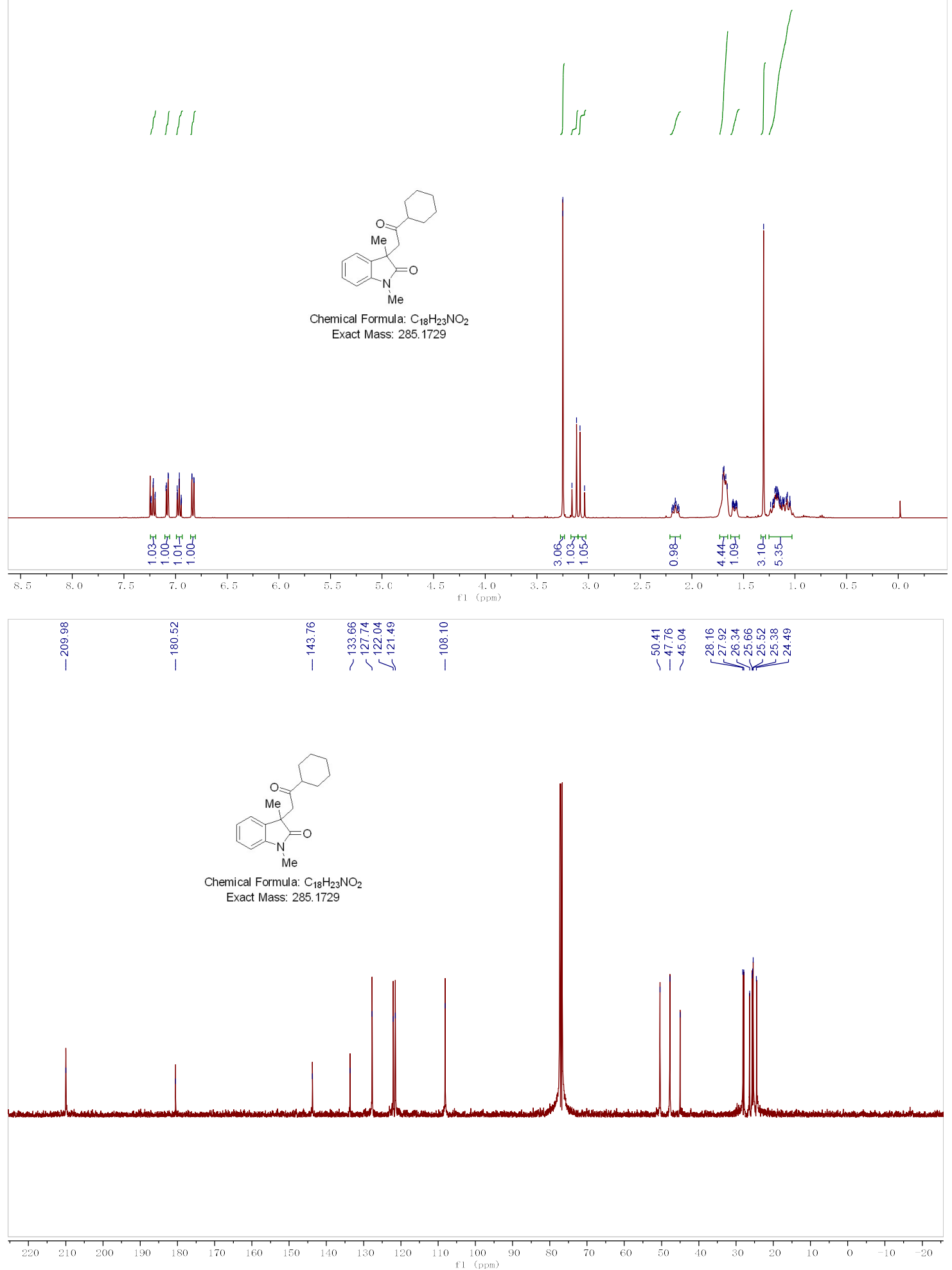
4ba

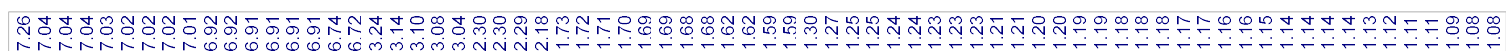

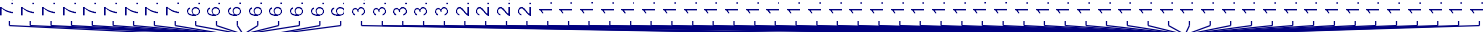

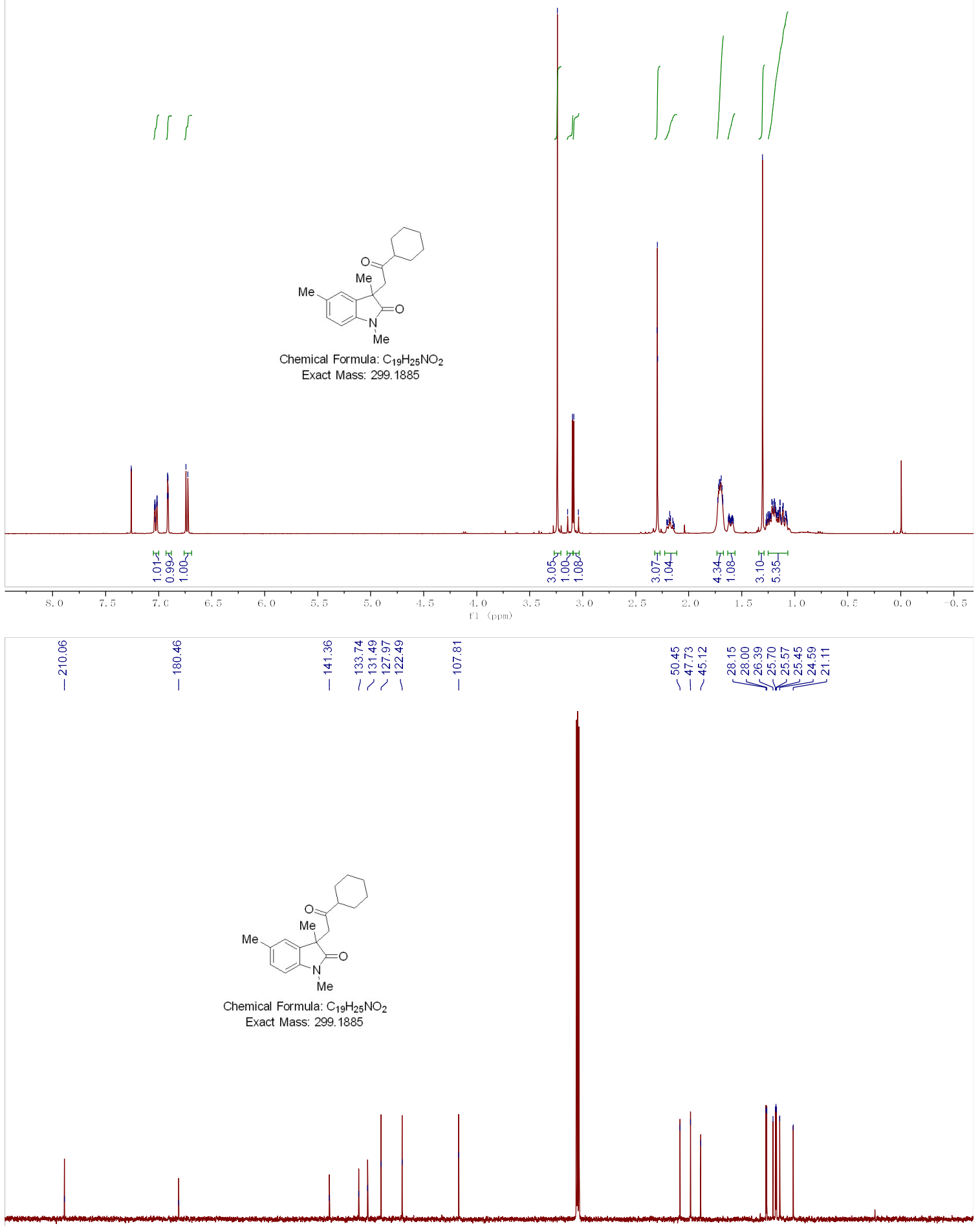

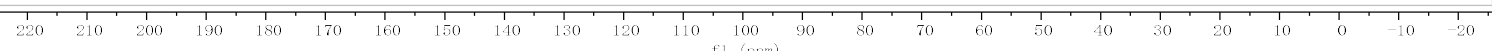




\section{4ca}

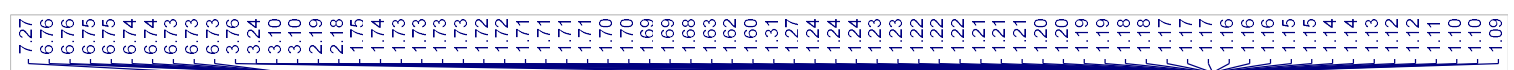
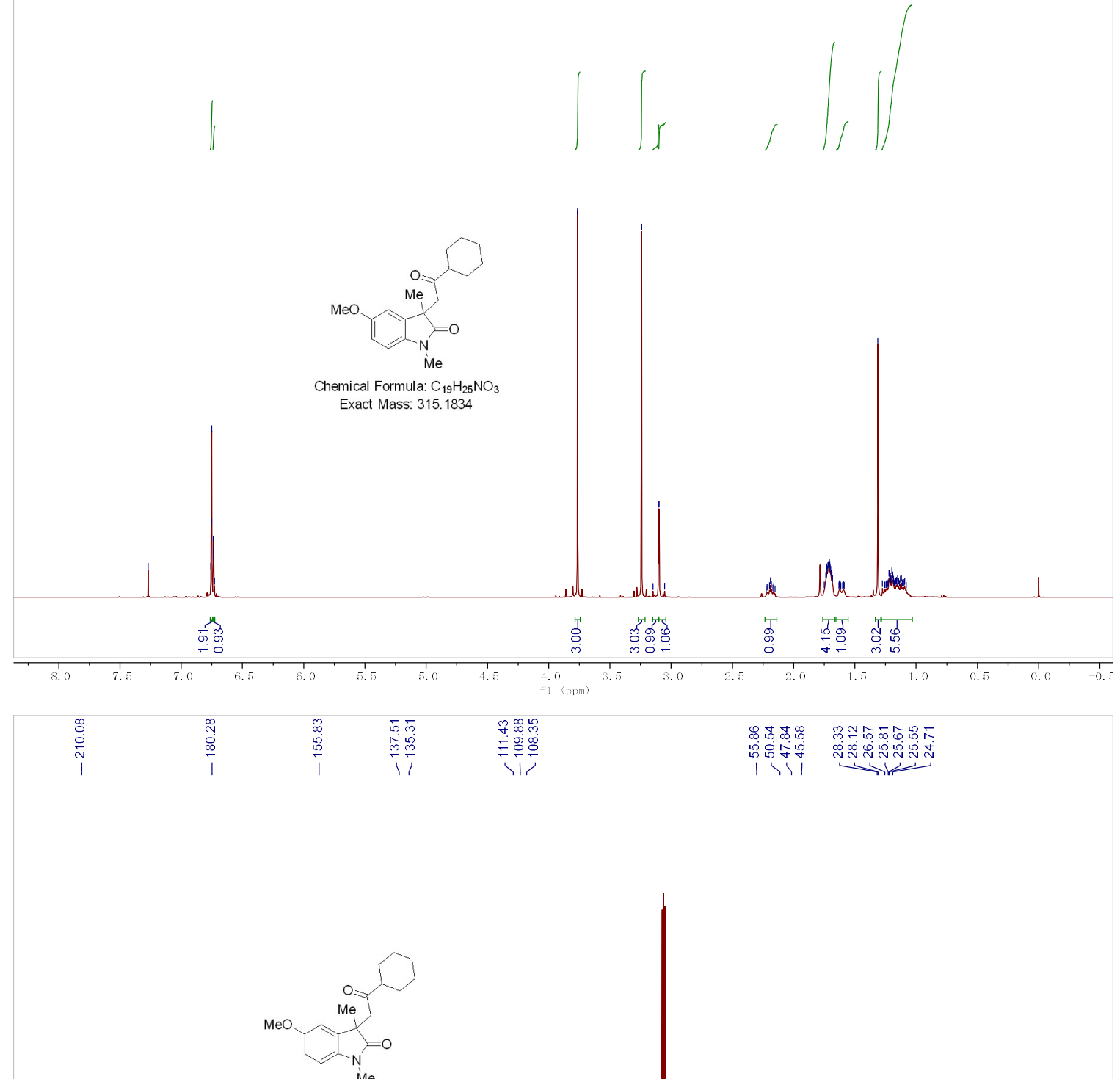

Chemical Formula: $\mathrm{C}_{19} \mathrm{H}_{25} \mathrm{NO}_{3}$

Exact Mass: 315.1834
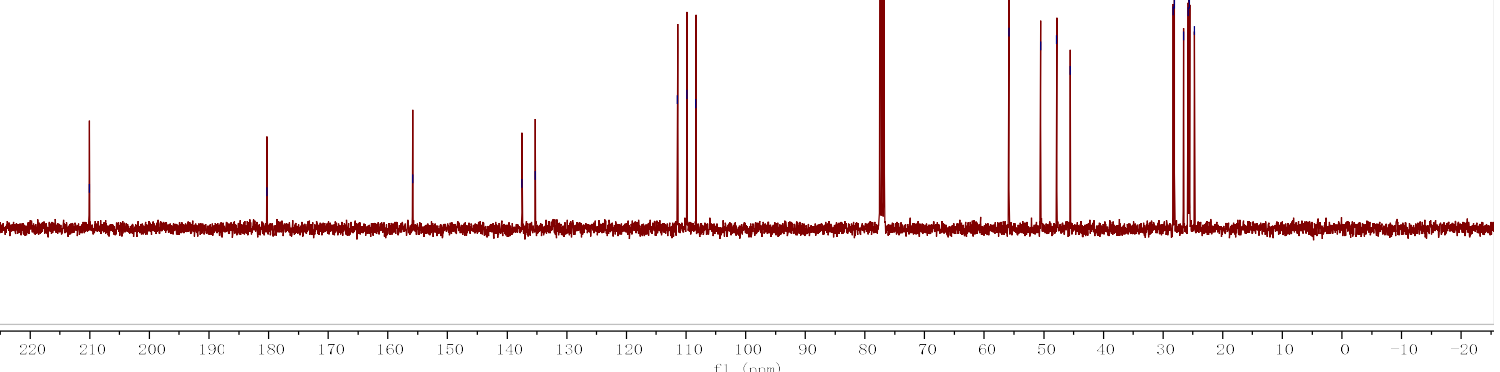


\section{4da}

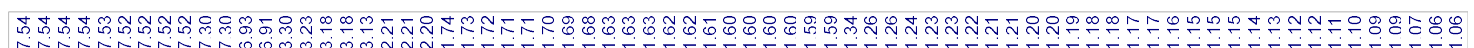

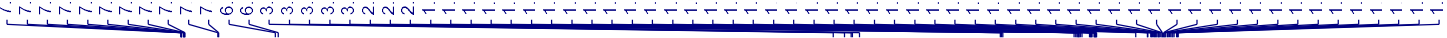
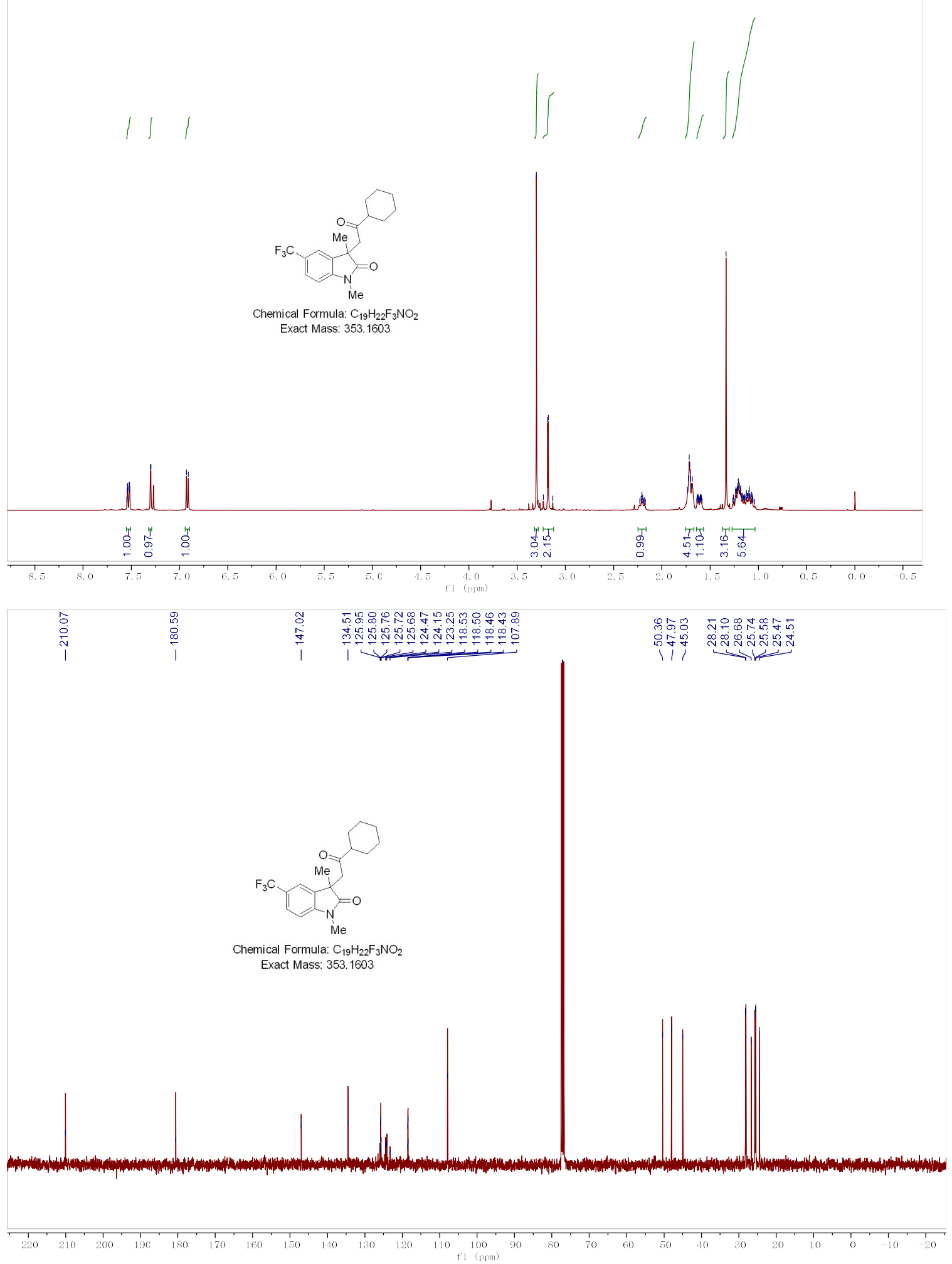


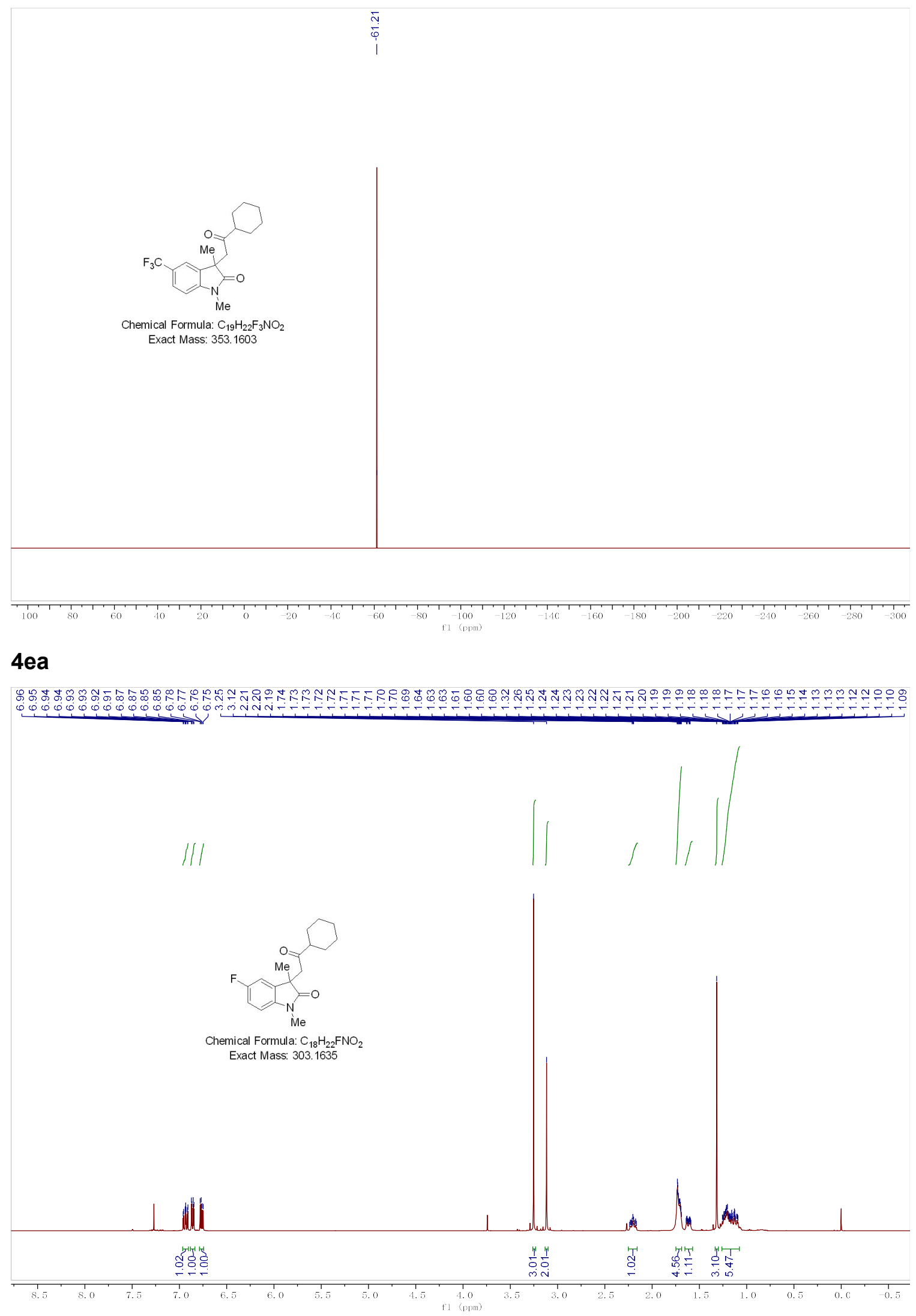



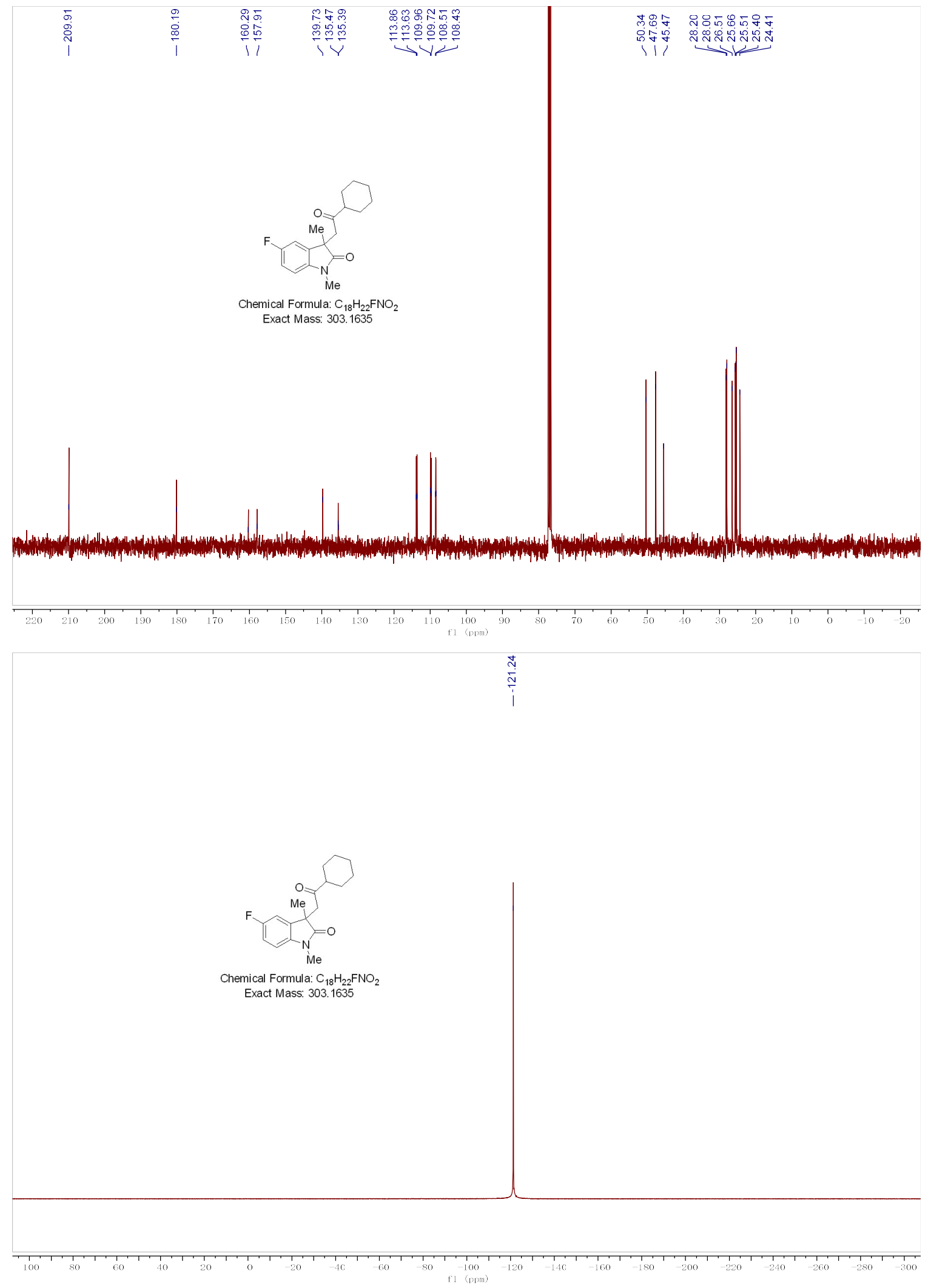

S39 


\section{$4 \mathrm{fa}$}

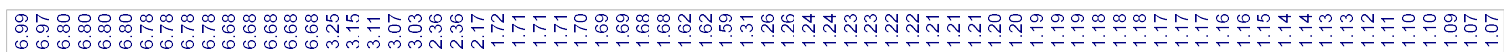
$000000000000000 \mathrm{mmmmmann}$
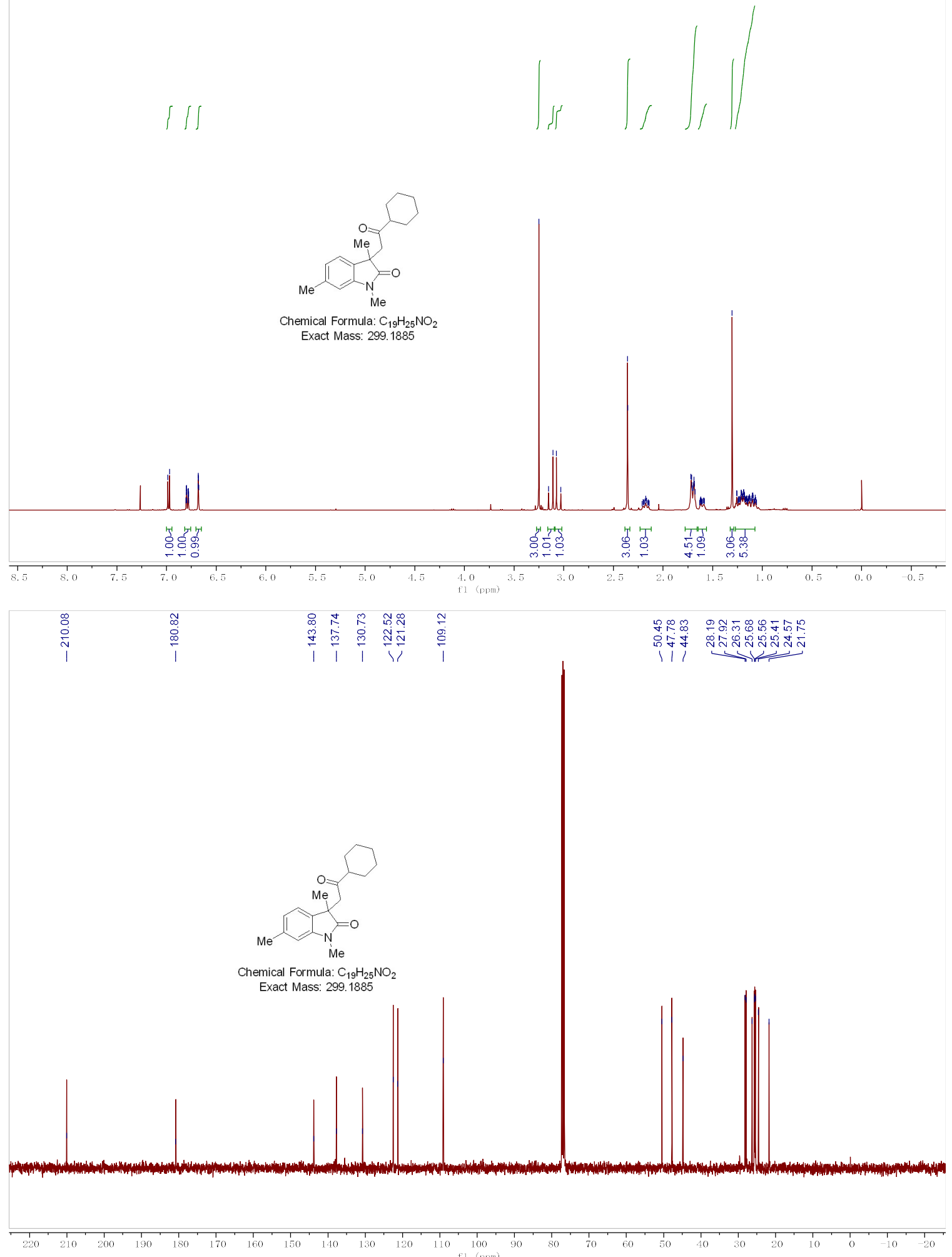


\section{4ga}

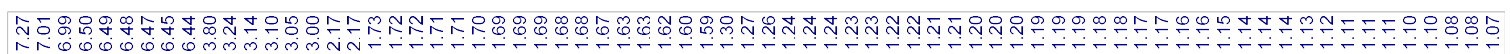

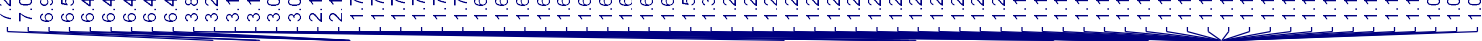
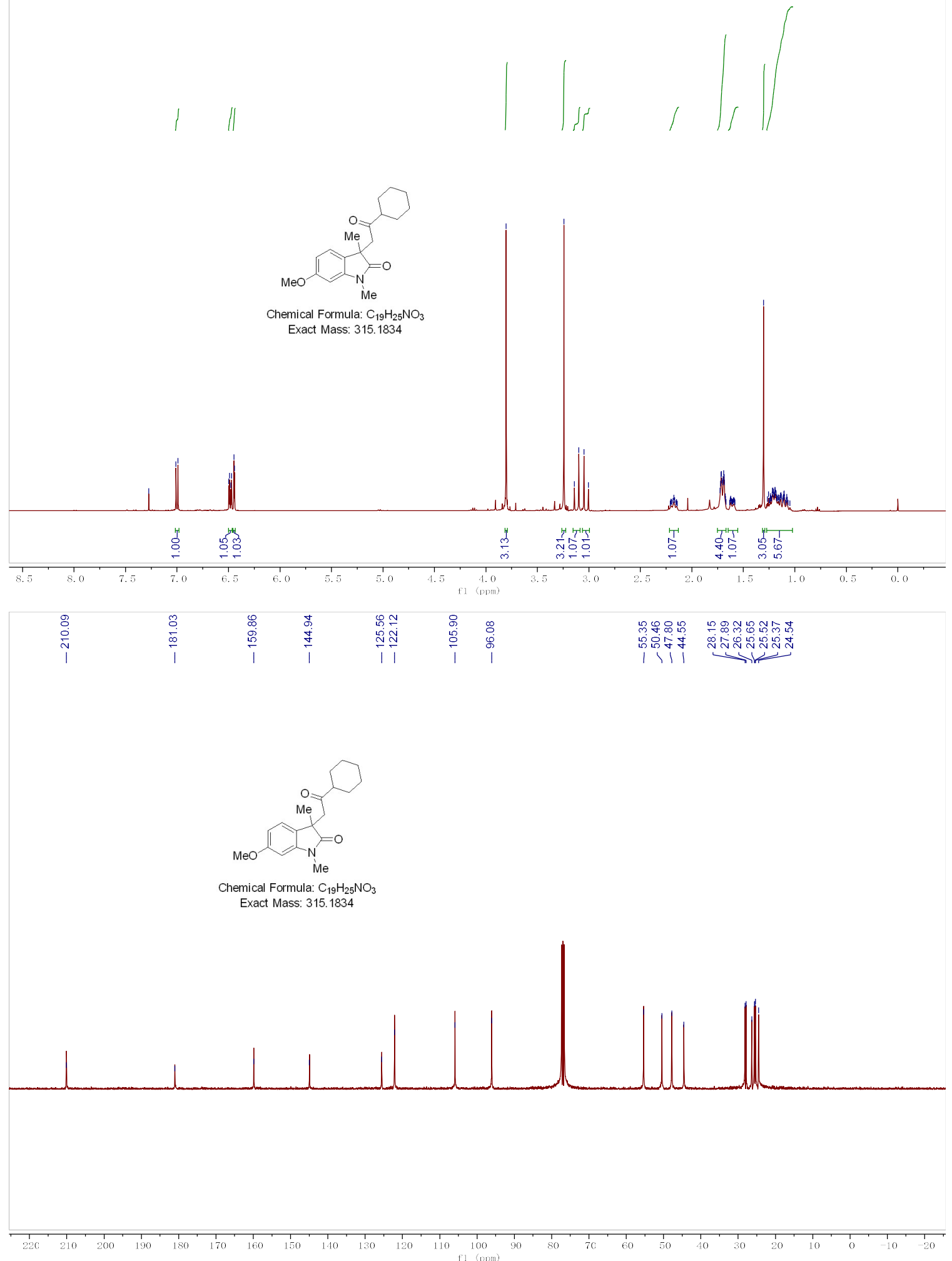


\section{4ha}

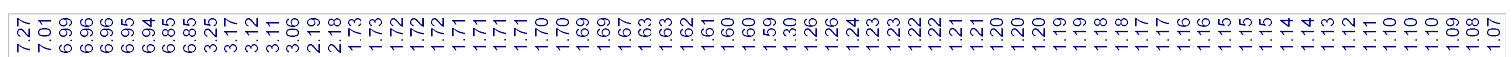

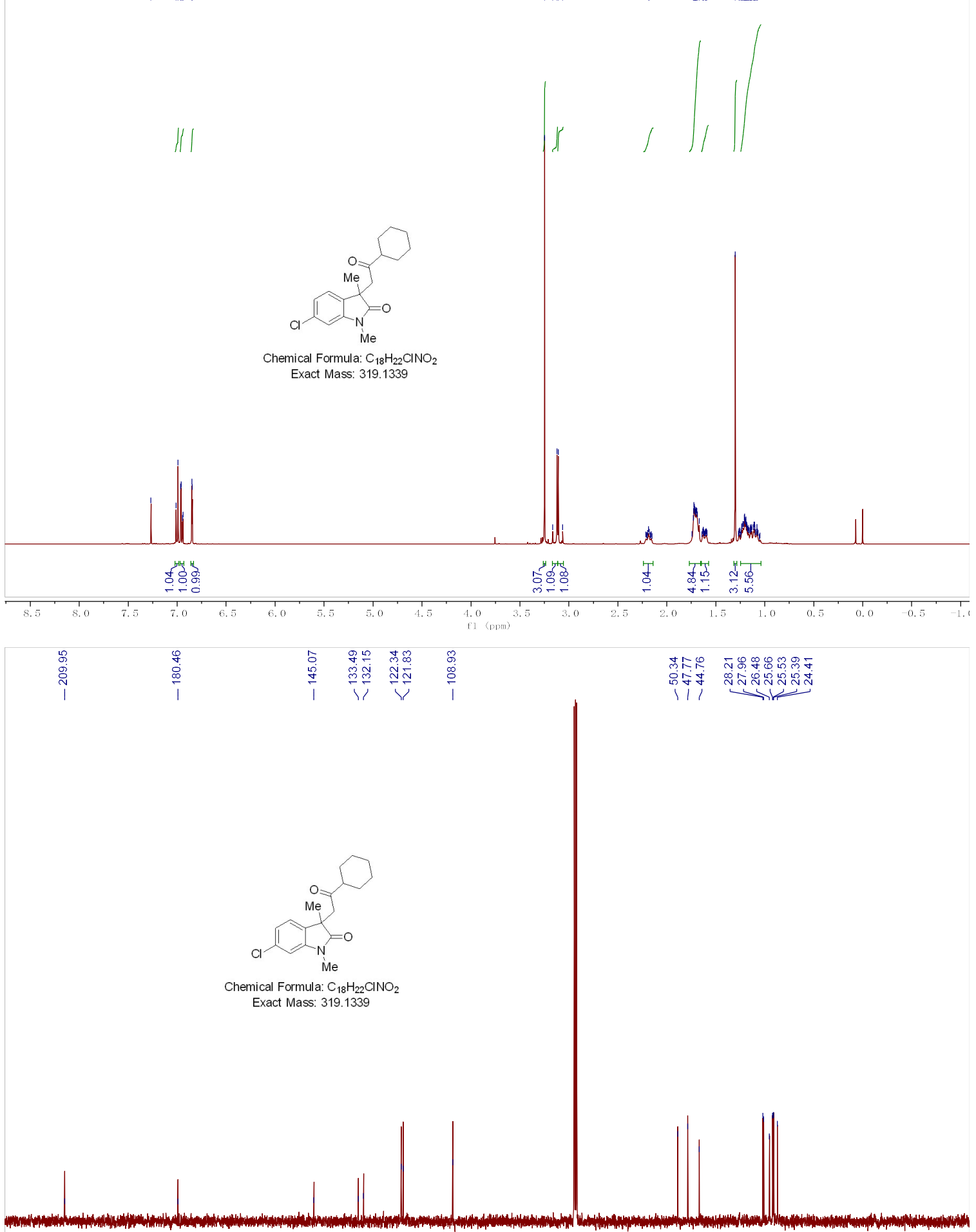

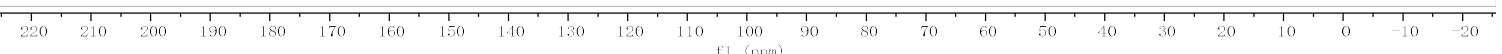


4ia

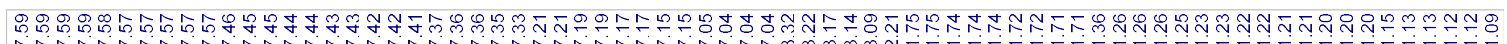

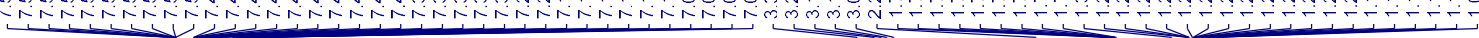

1111
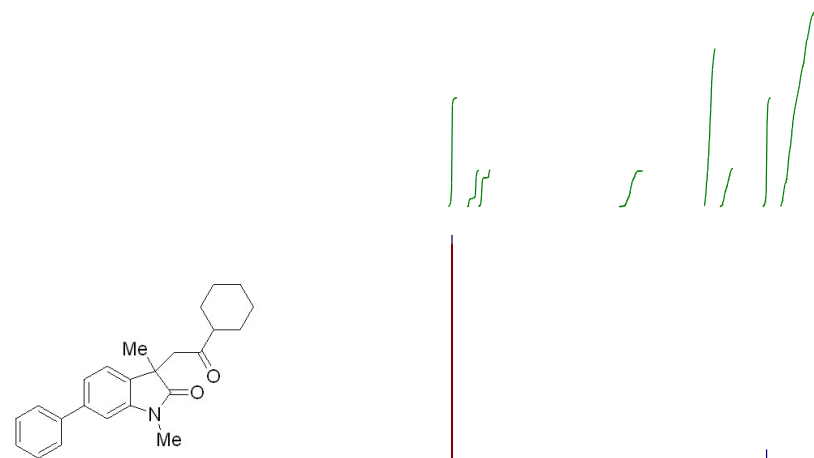

Chemical Formula: $\mathrm{C}_{24} \mathrm{H}_{27} \mathrm{NO}_{2}$ Exact Mass: 361.2042
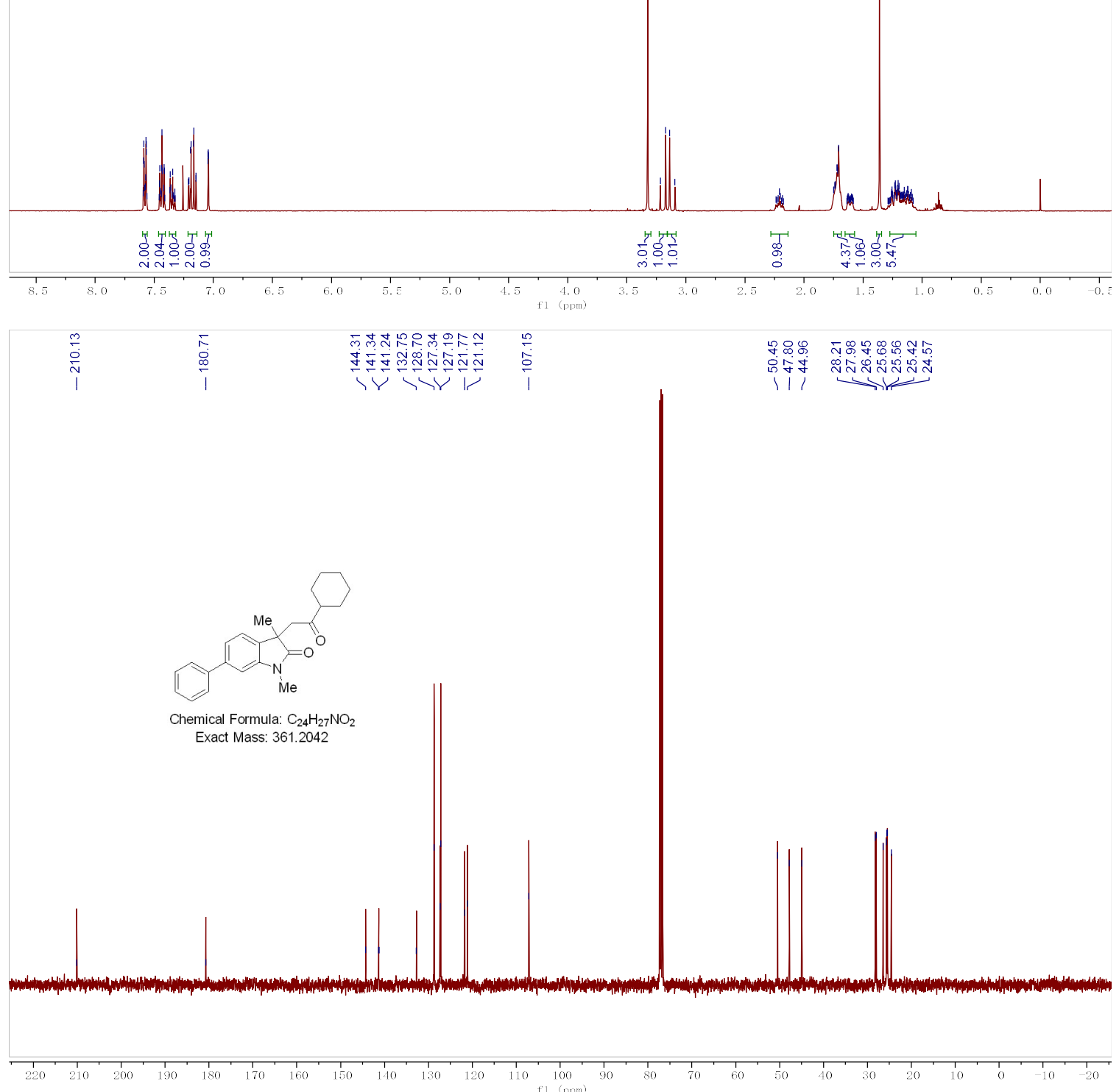


\section{$4 \mathrm{ja}$}

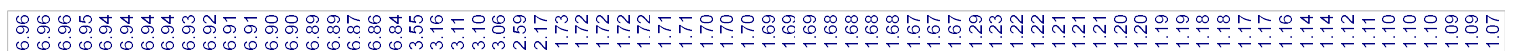

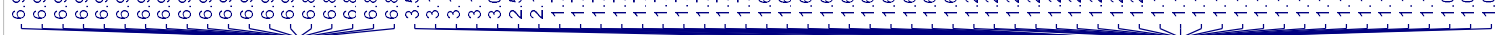

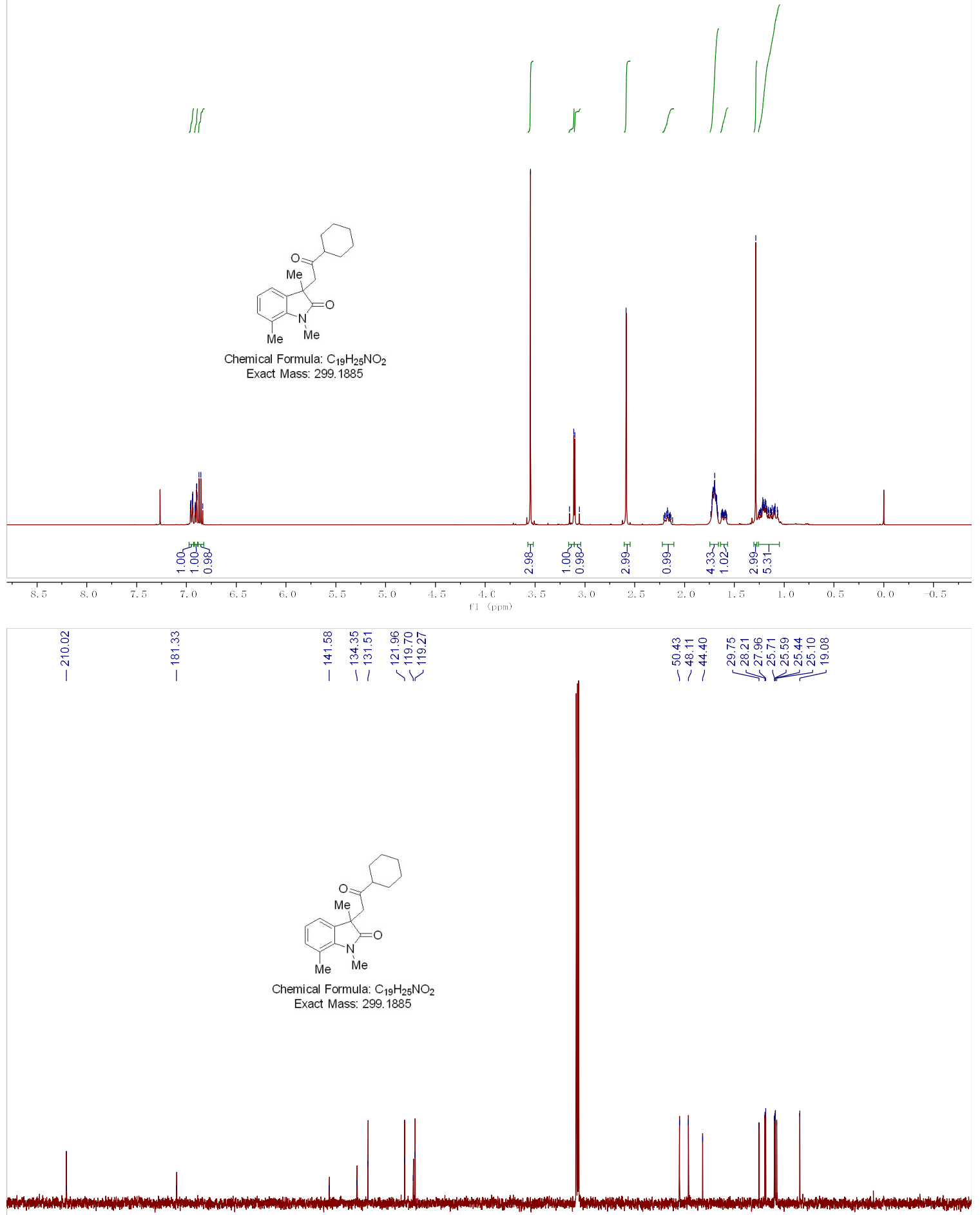

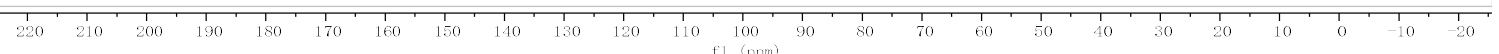




\section{4ka}

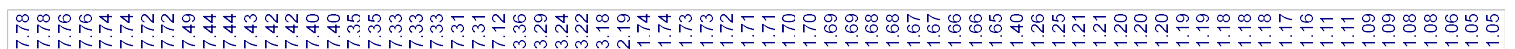

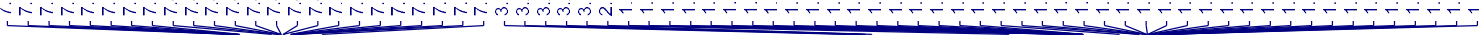
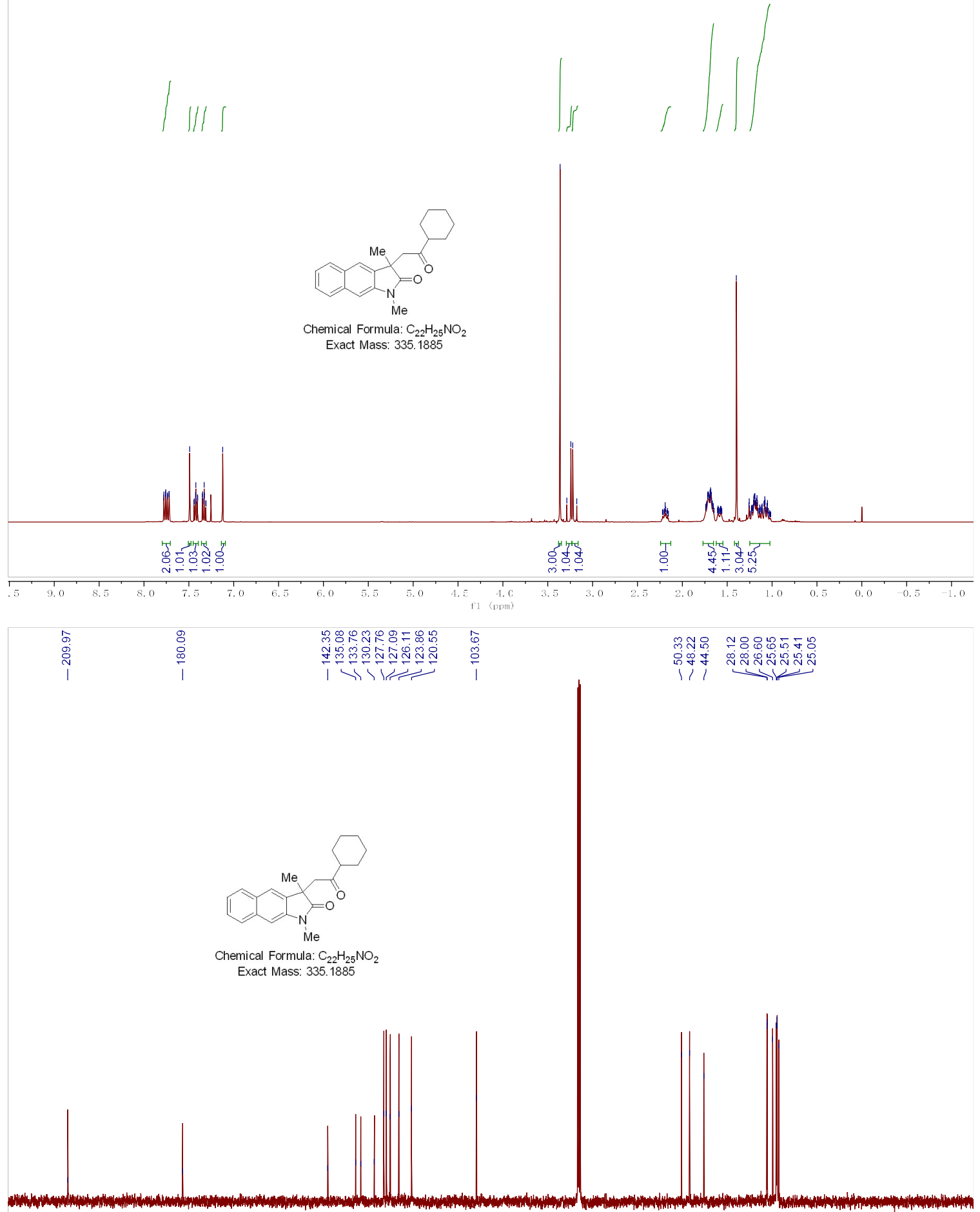

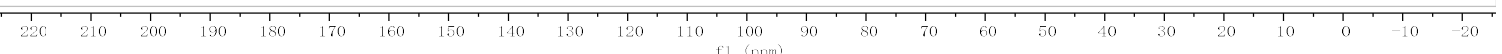


$4 \mathrm{la}$

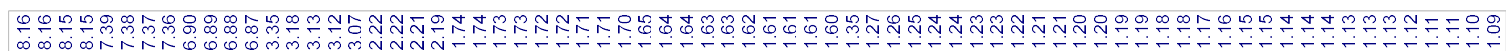

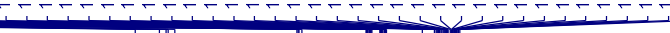

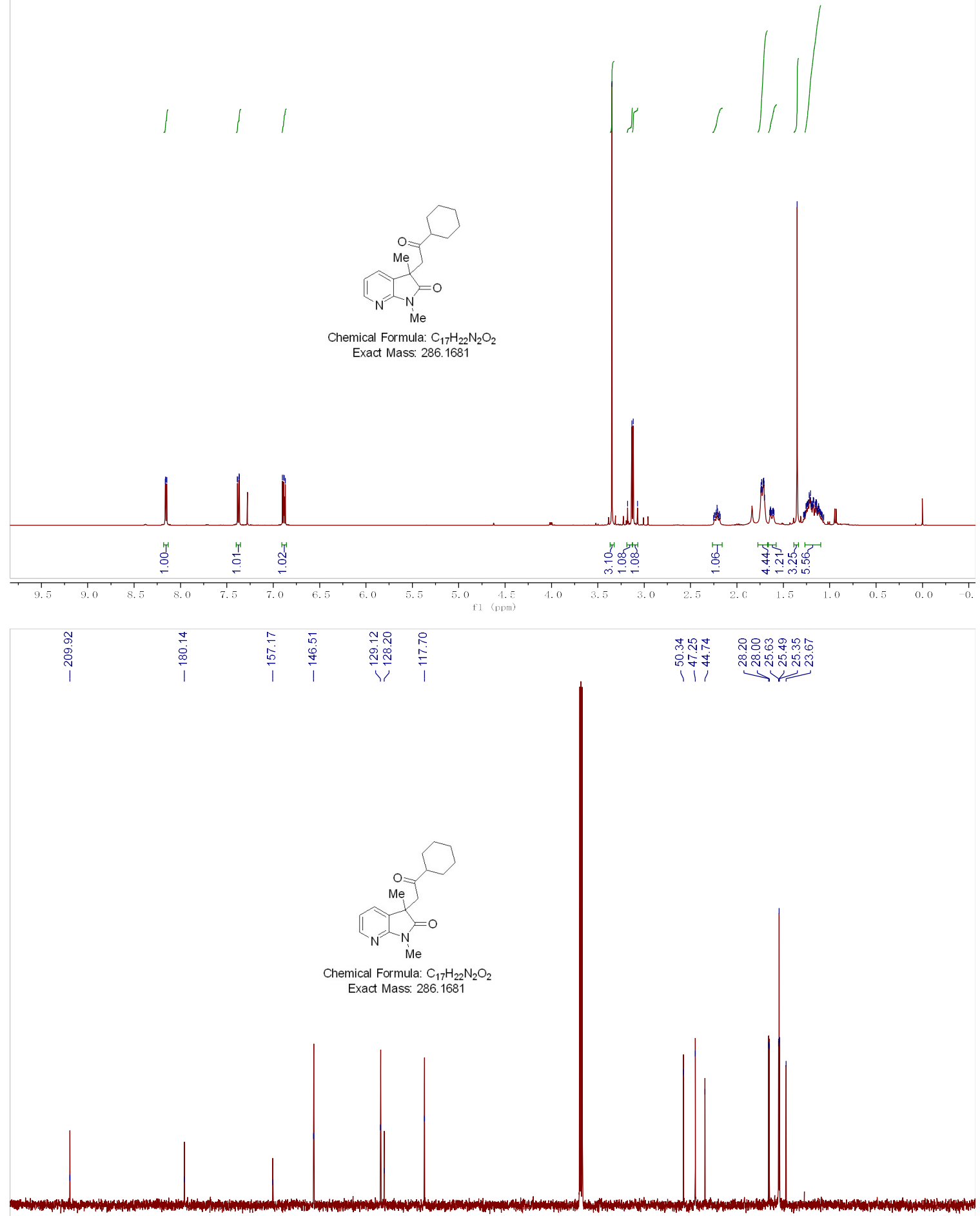

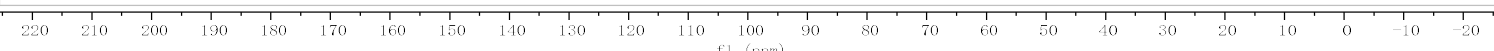




\section{4ma}

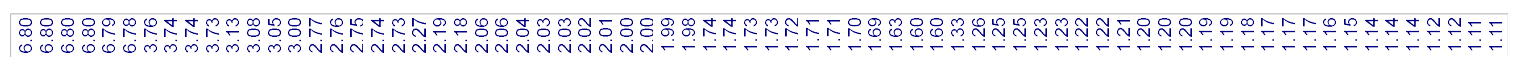

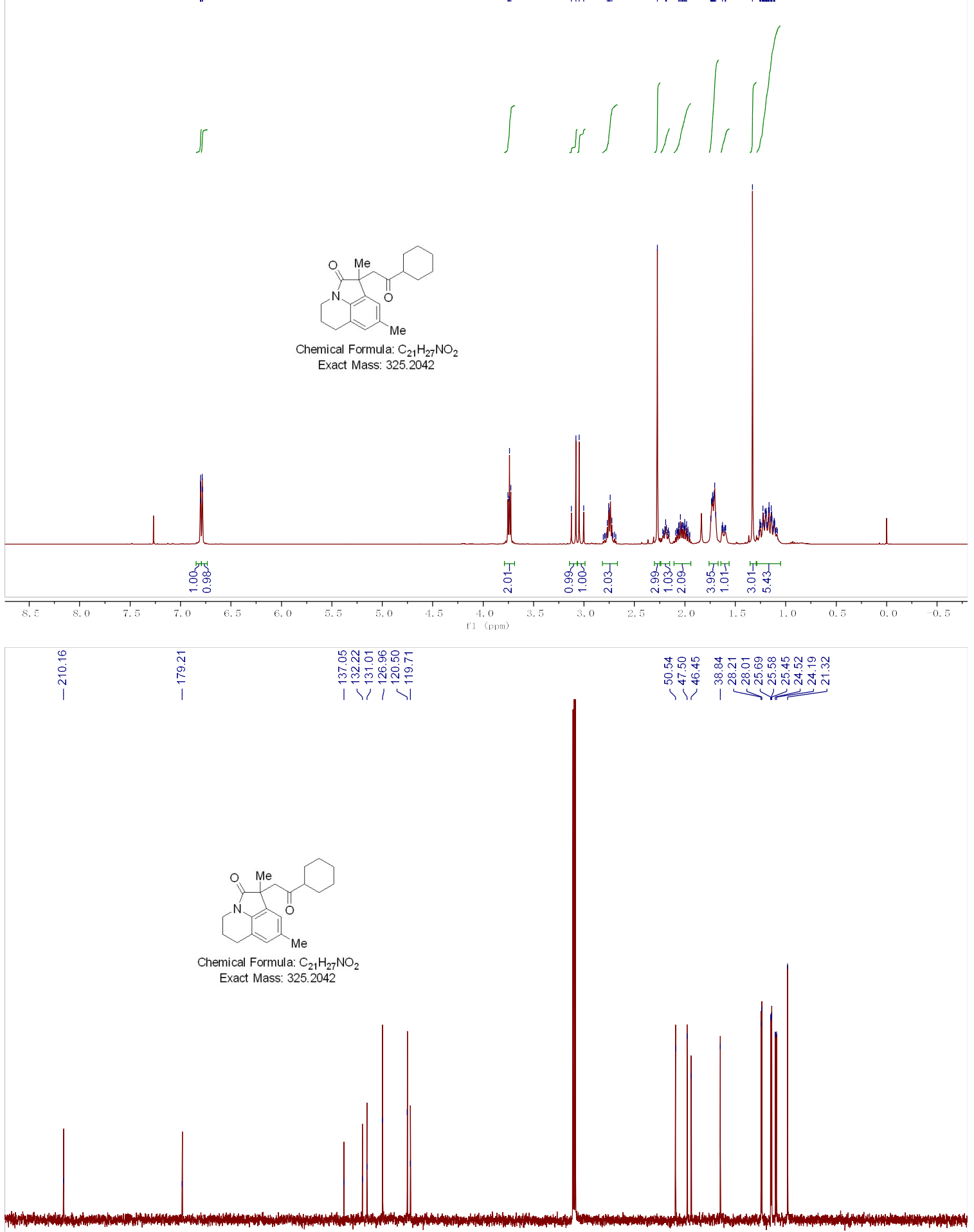

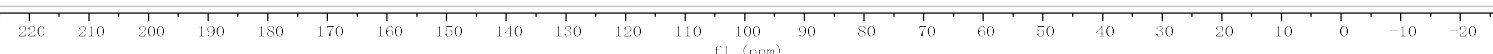




\section{4na}

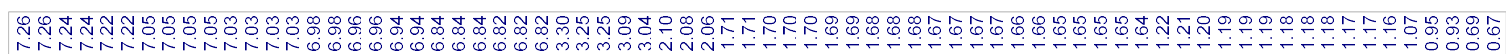

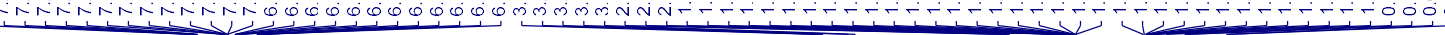
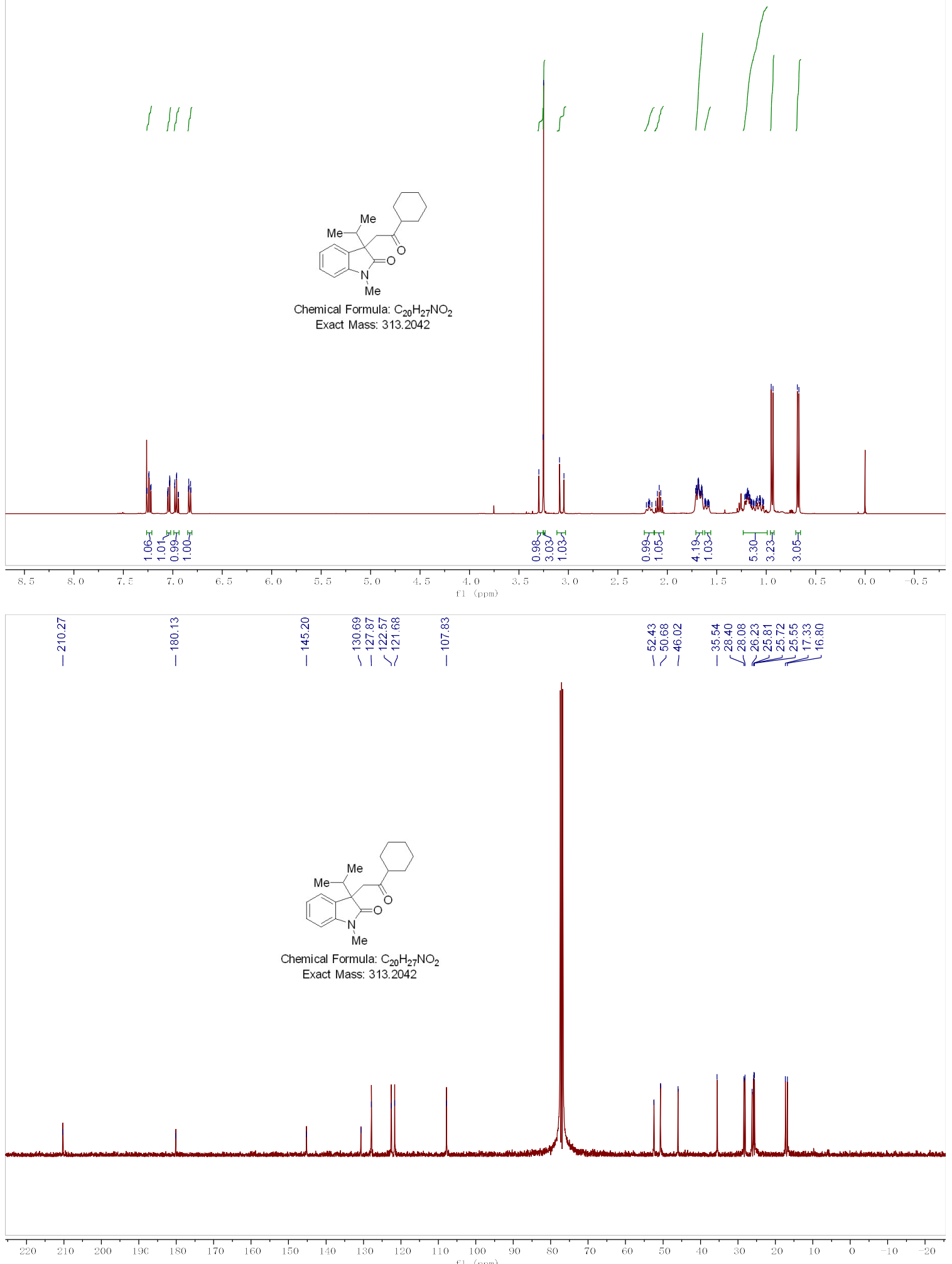
$40 a$

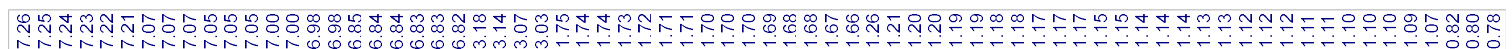

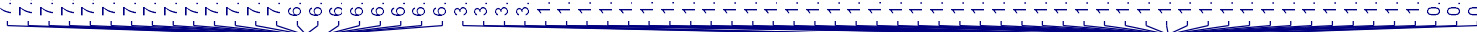

If 1

$\int 4$
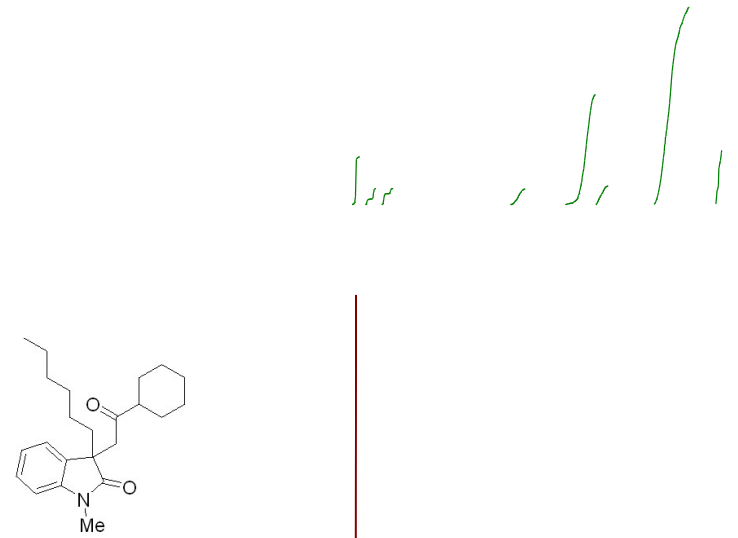

Chemical Formula: $\mathrm{C}_{23} \mathrm{H}_{33} \mathrm{NO}_{2}$

Exact Mass: 355.2511
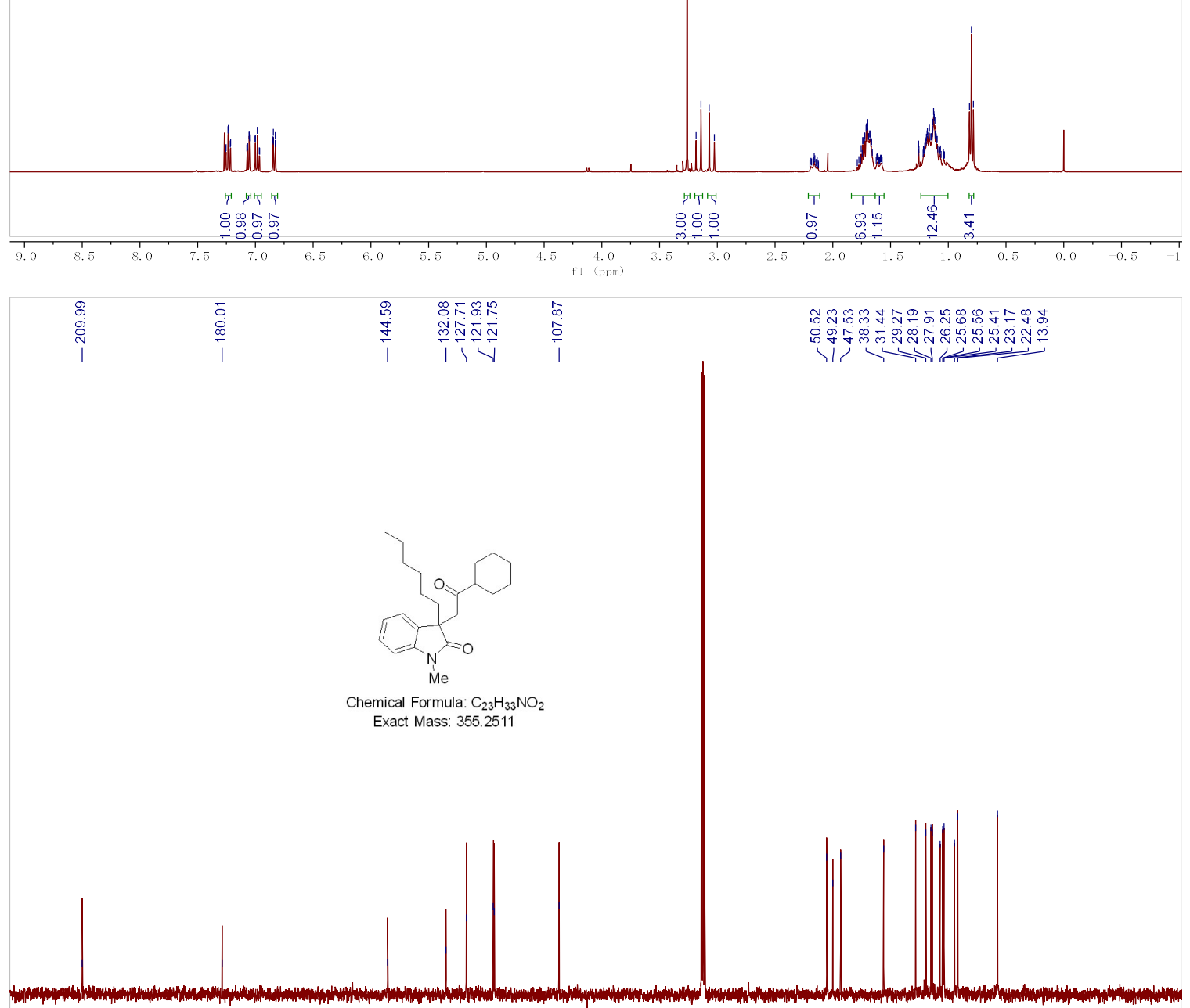

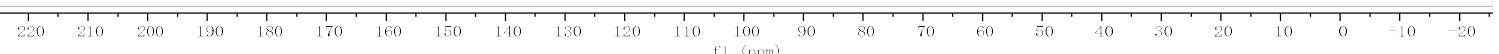




\section{$4 \mathrm{pa}$}

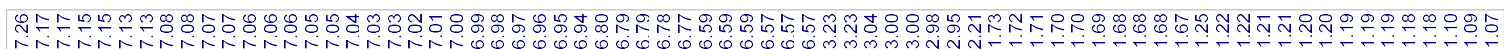

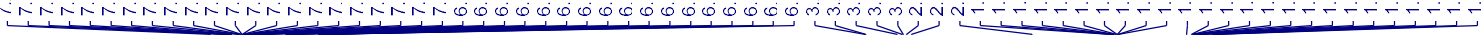
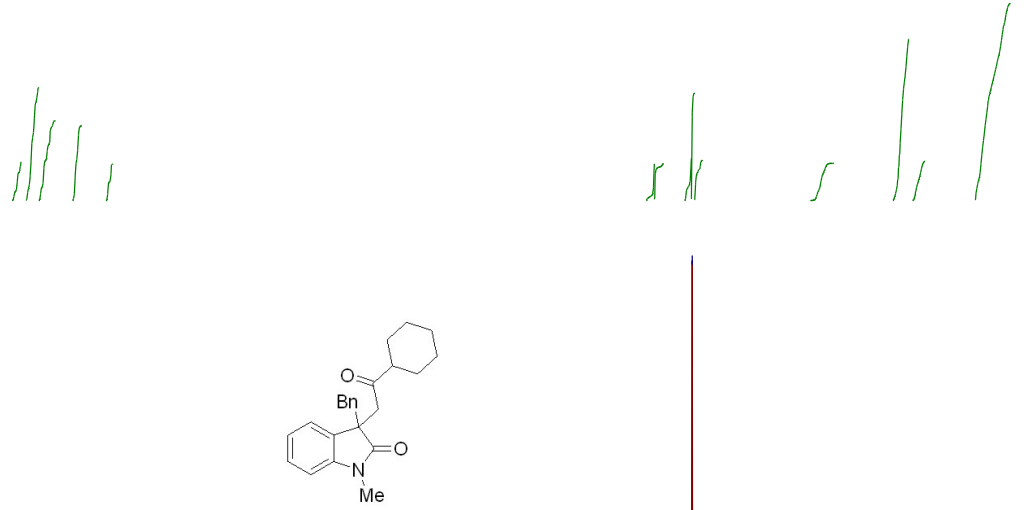

Chemical Formula: $\mathrm{C}_{24} \mathrm{H}_{27} \mathrm{NO}_{2}$

Exact Mass: 361.2042
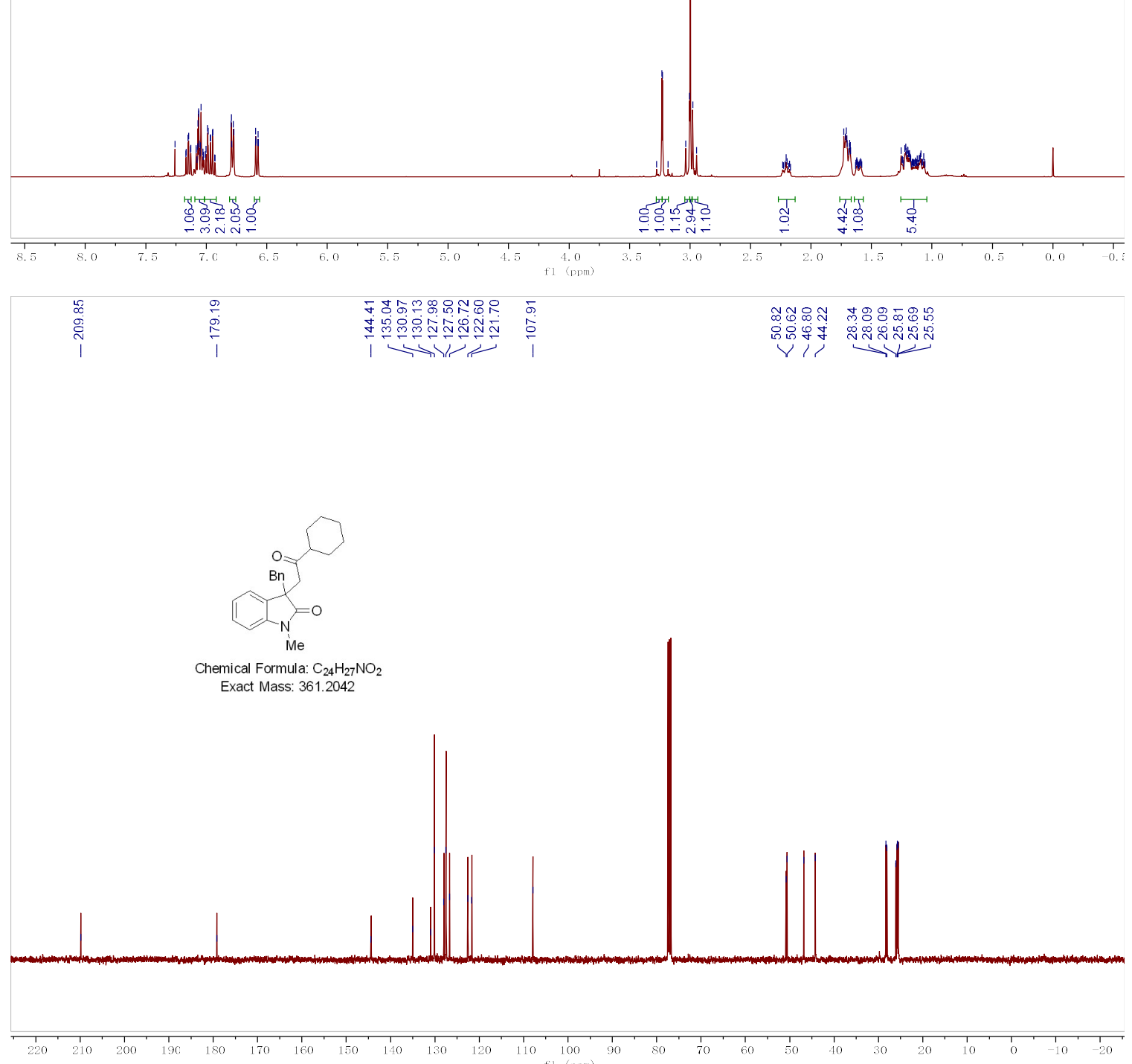
4qa

Dow

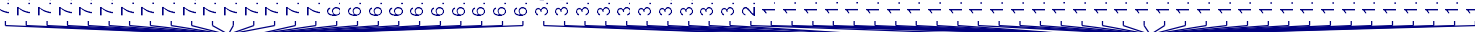
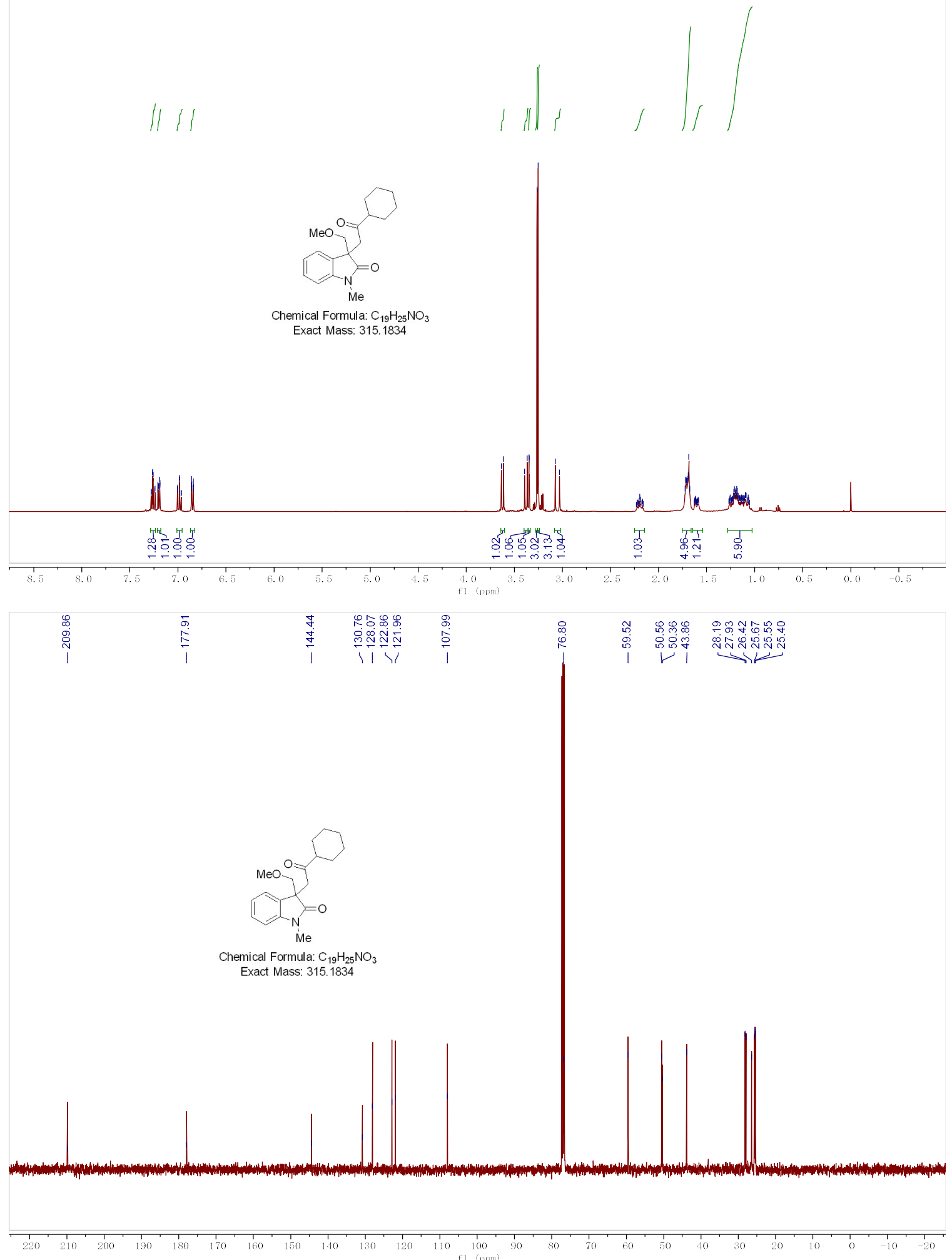


\section{4ra}

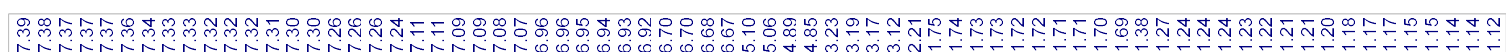

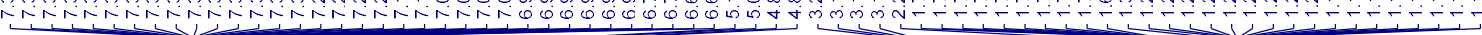
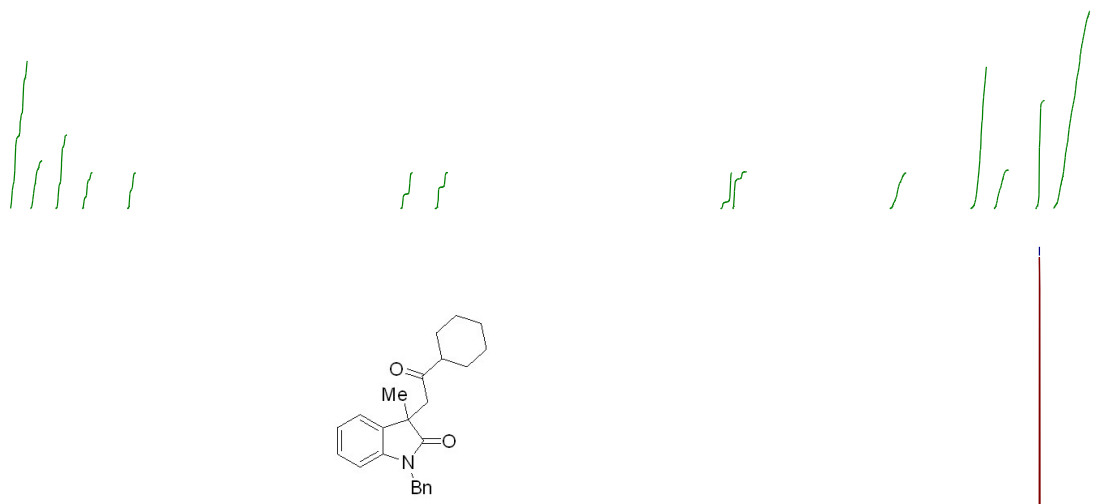

Chemical Formula: $\mathrm{C}_{24} \mathrm{H}_{27} \mathrm{NO}_{2}$

Exact Mass: 361.2042
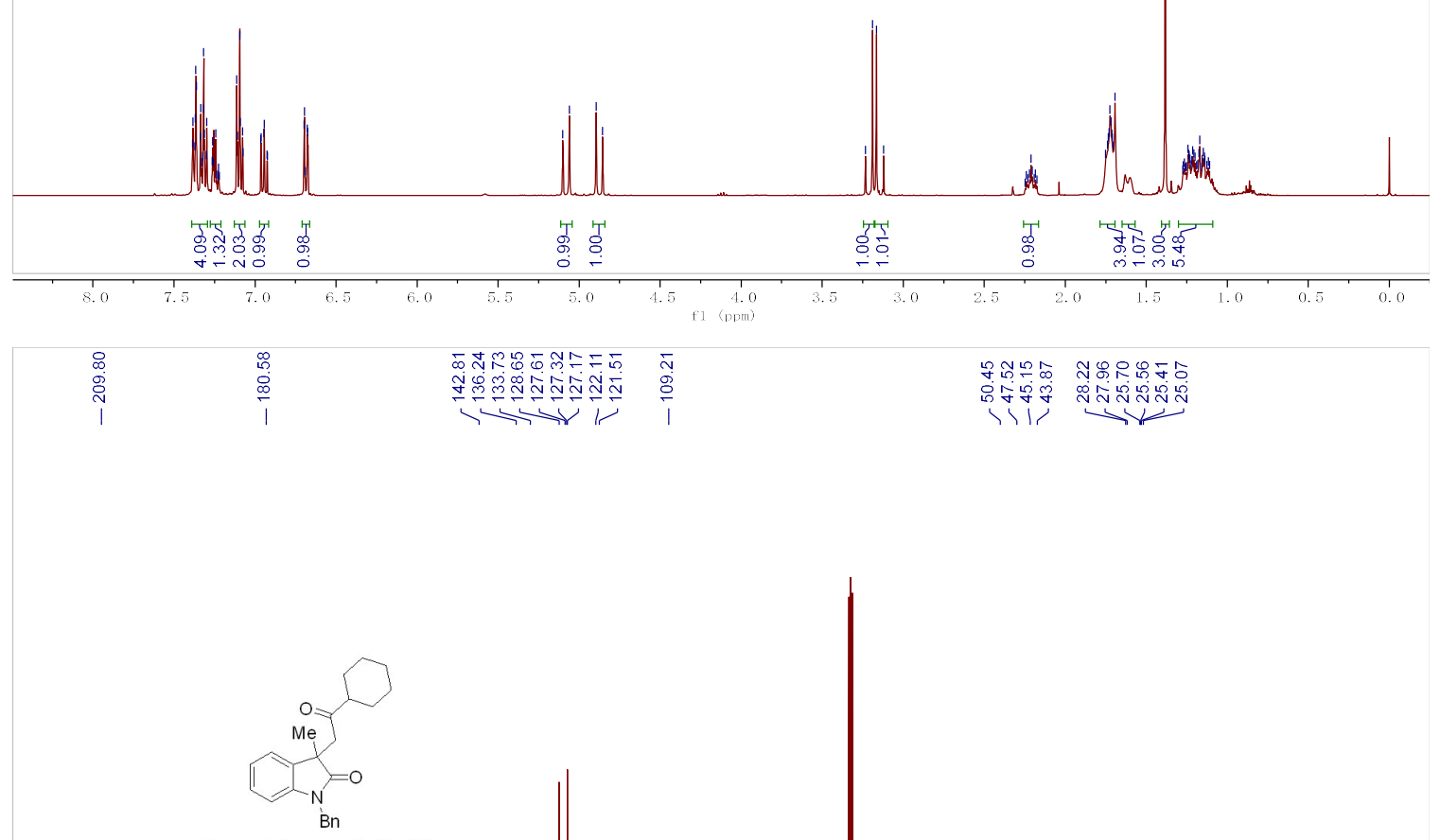

Chemical Formula: $\mathrm{C}_{24} \mathrm{H}_{27} \mathrm{NO}_{2}$

Exact Mass: 361.2042
-
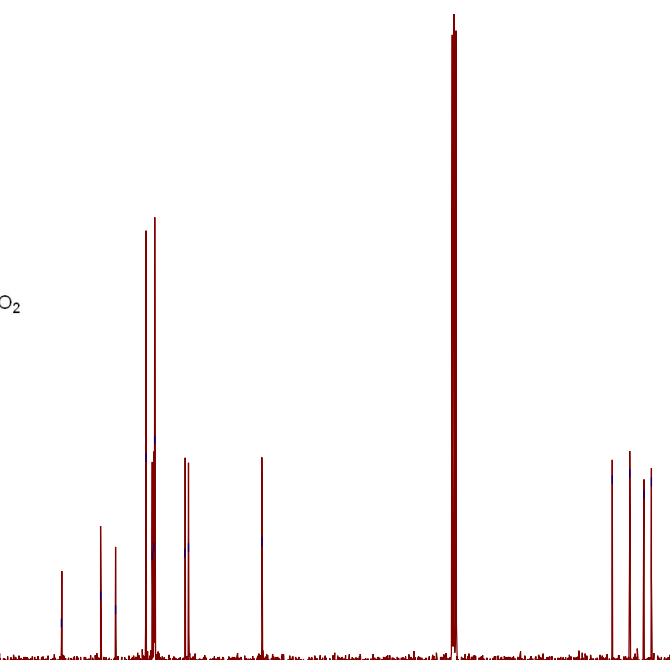

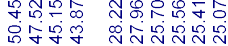

1,10

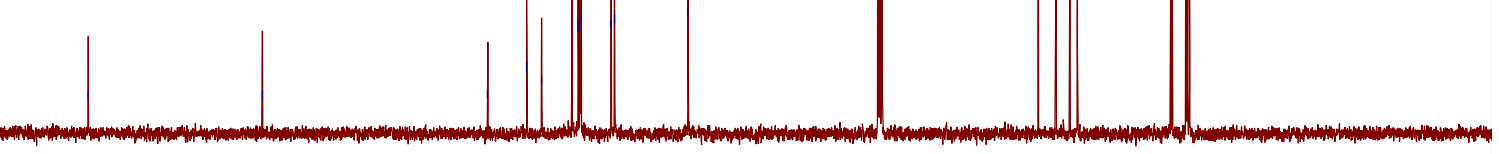




\section{4ab}

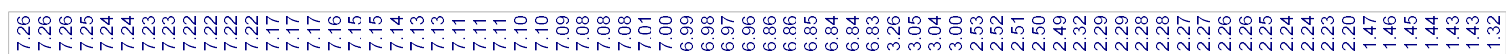

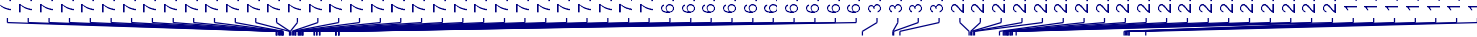

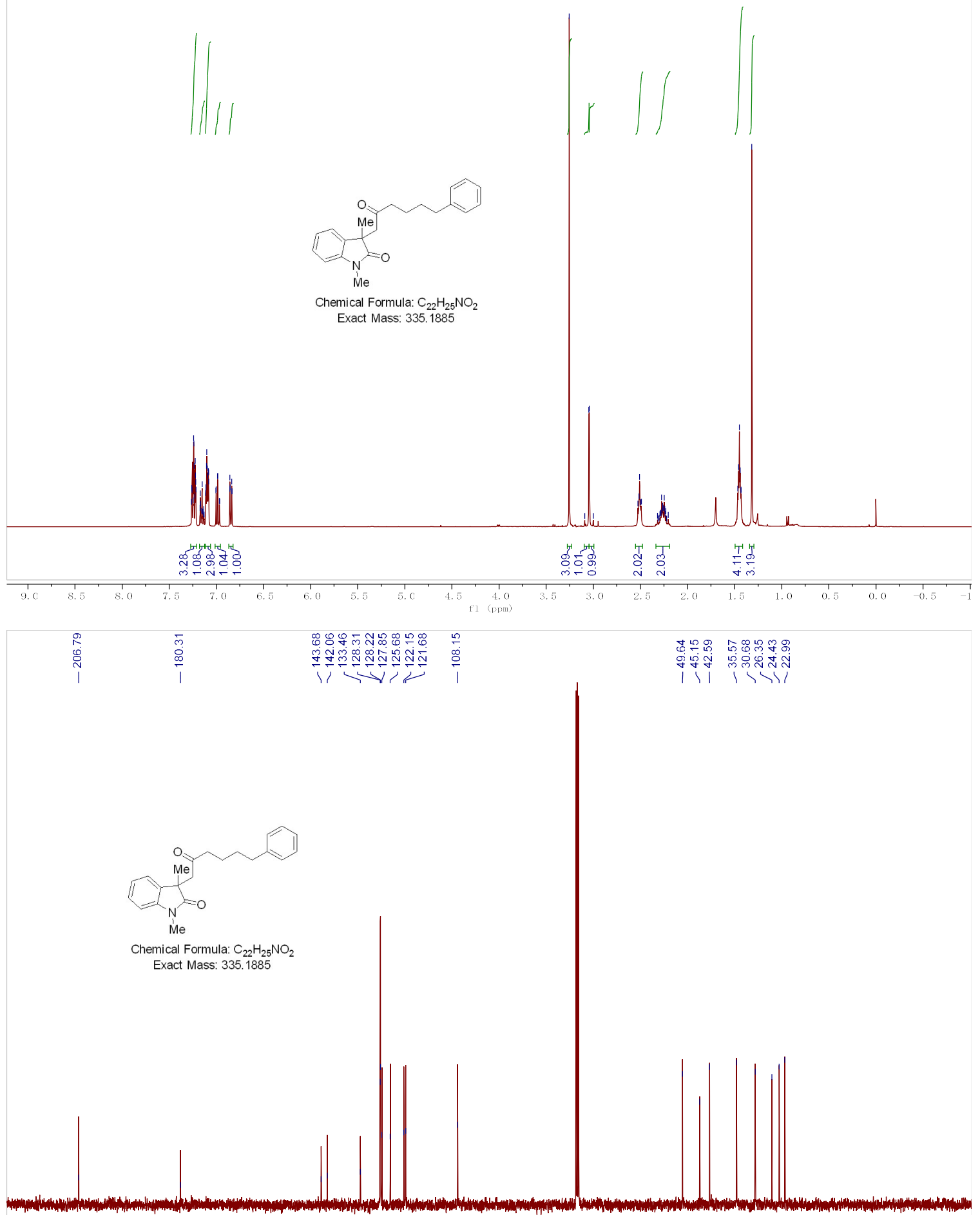

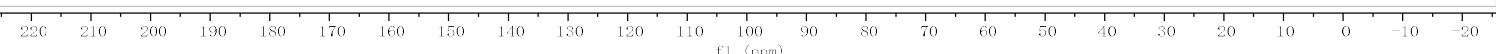


$4 a c$

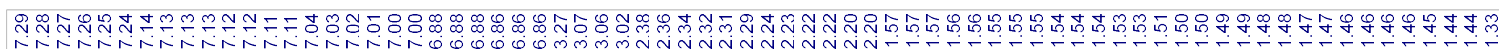

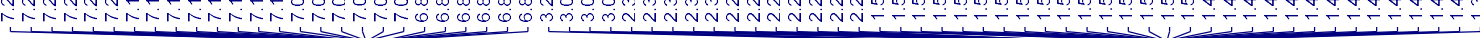
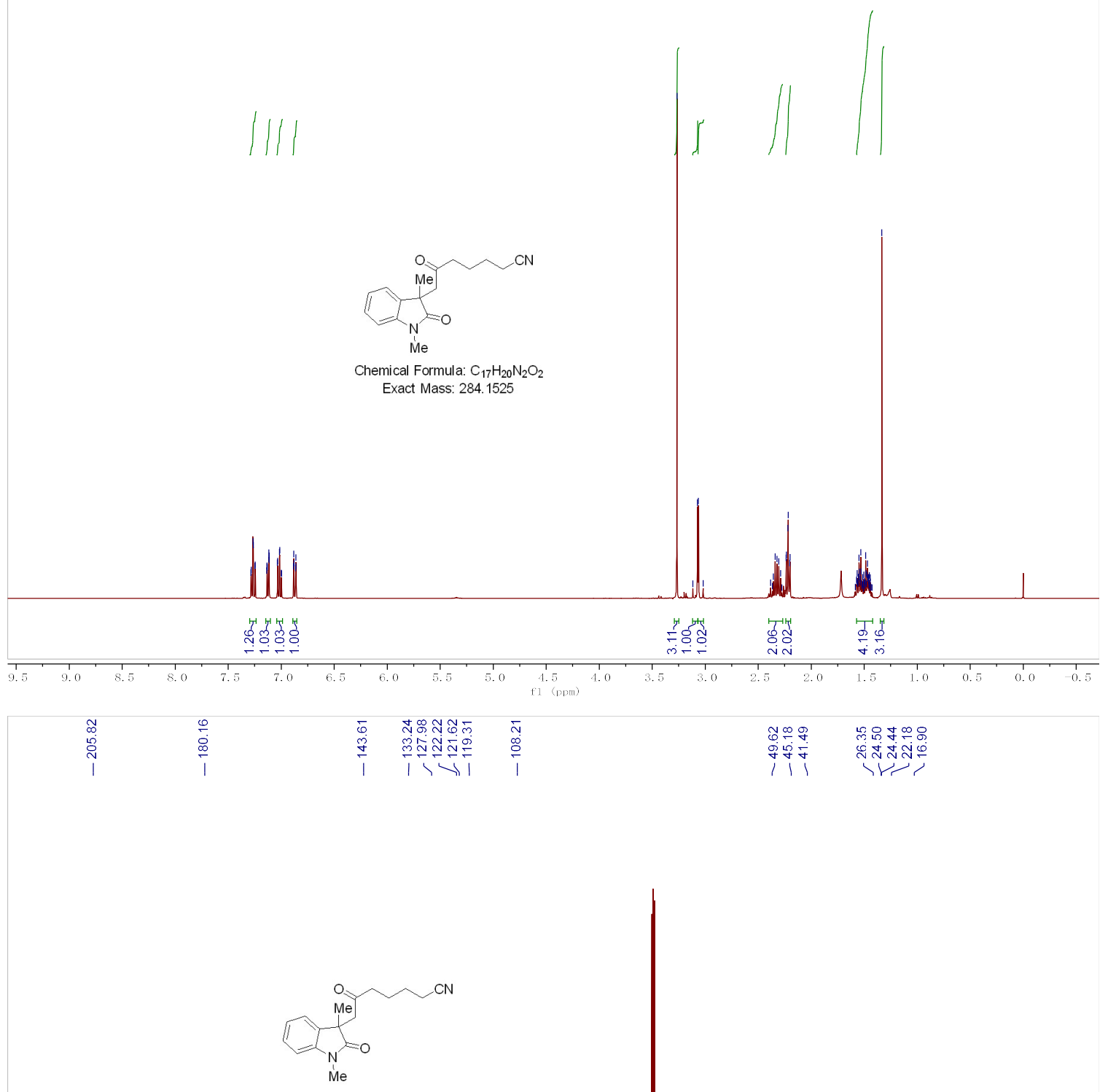

Chemical Formula: $\mathrm{C}_{17} \mathrm{H}_{20} \mathrm{~N}_{2} \mathrm{O}_{2}$ Exact Mass: 284.1525
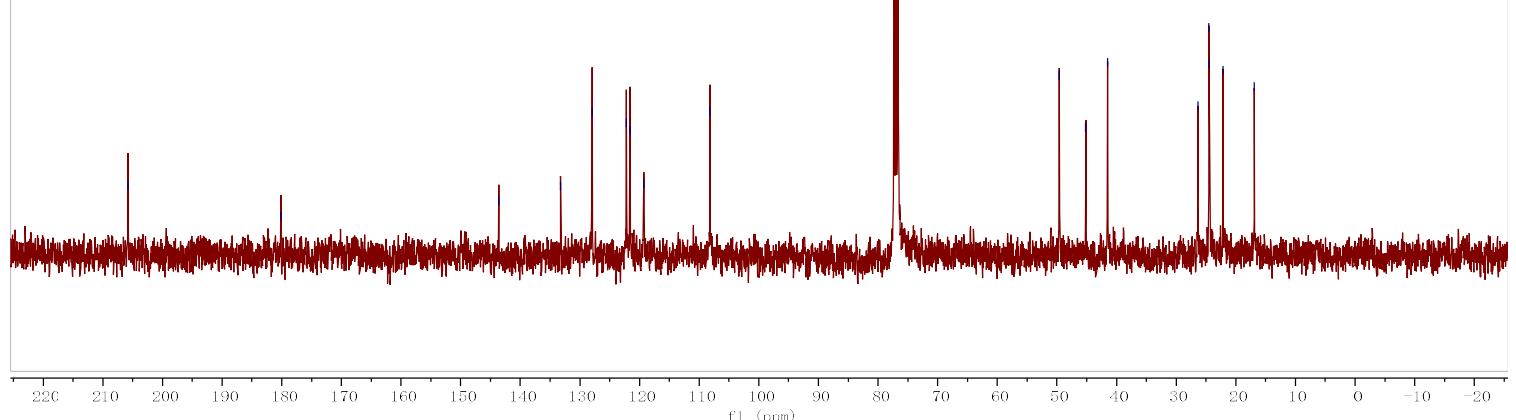


\section{4ad}
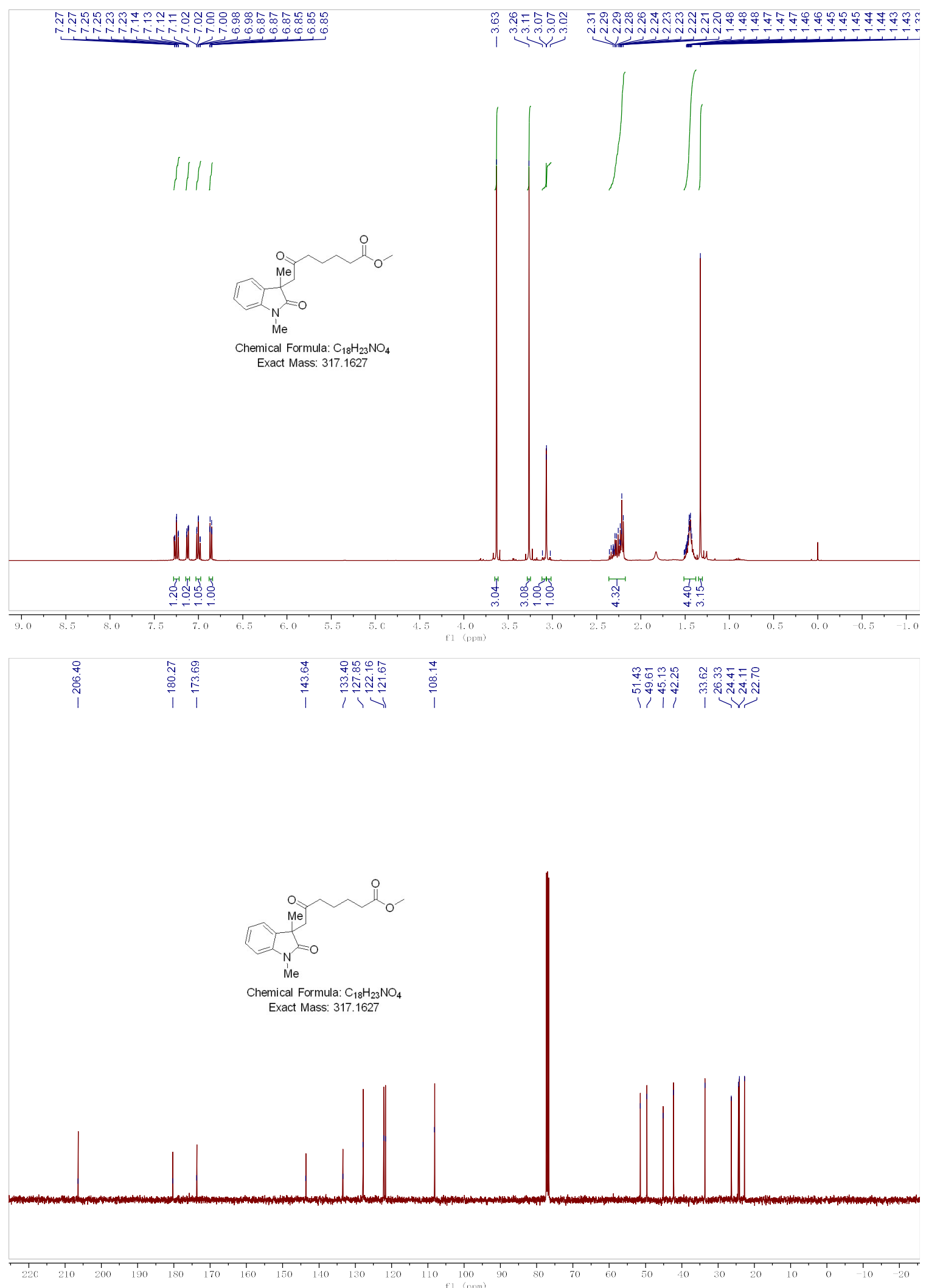


\section{$4 a e$}

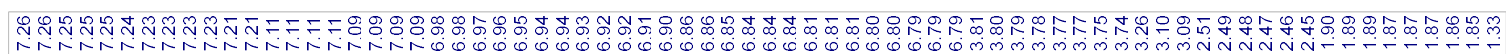
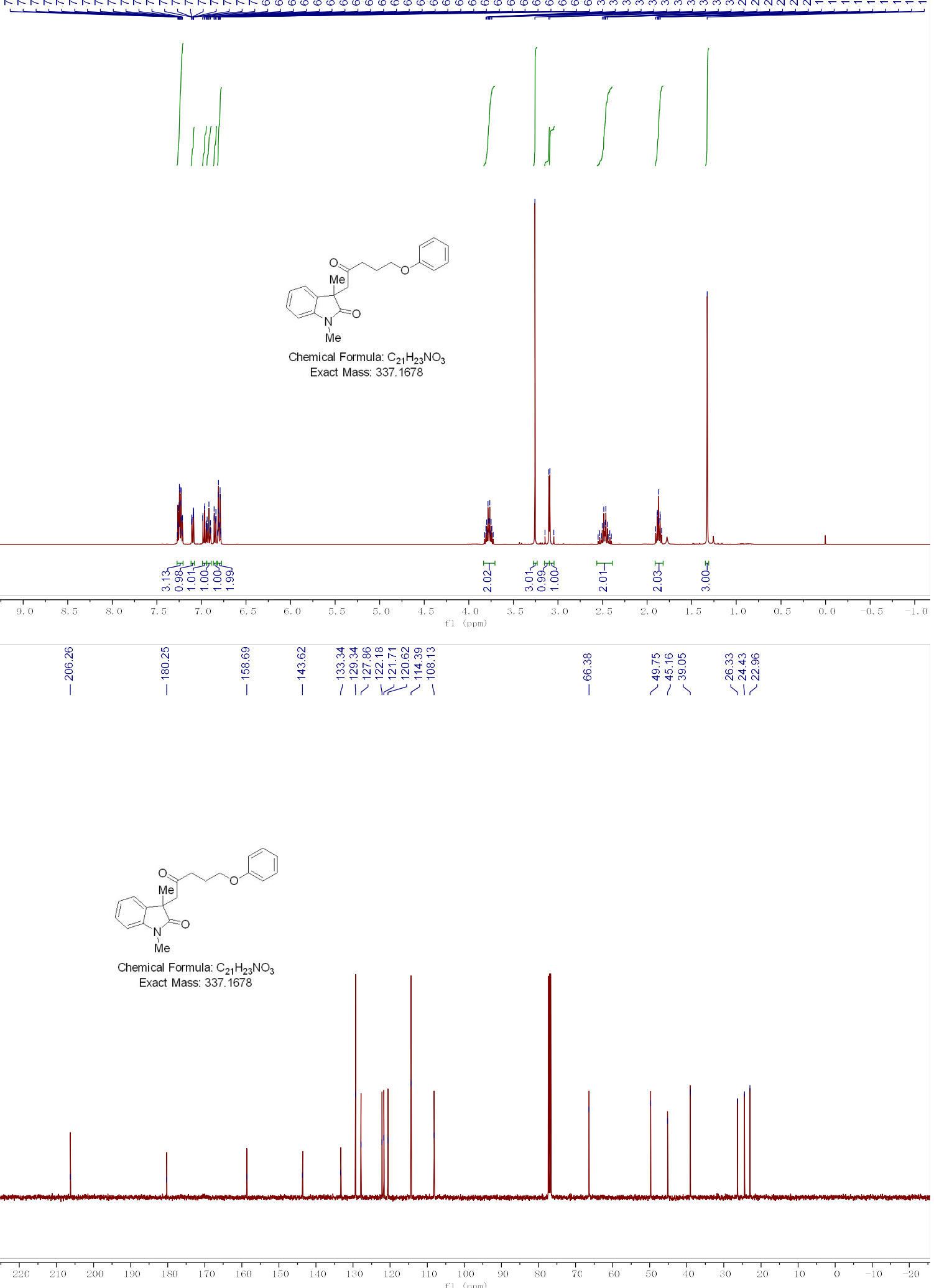

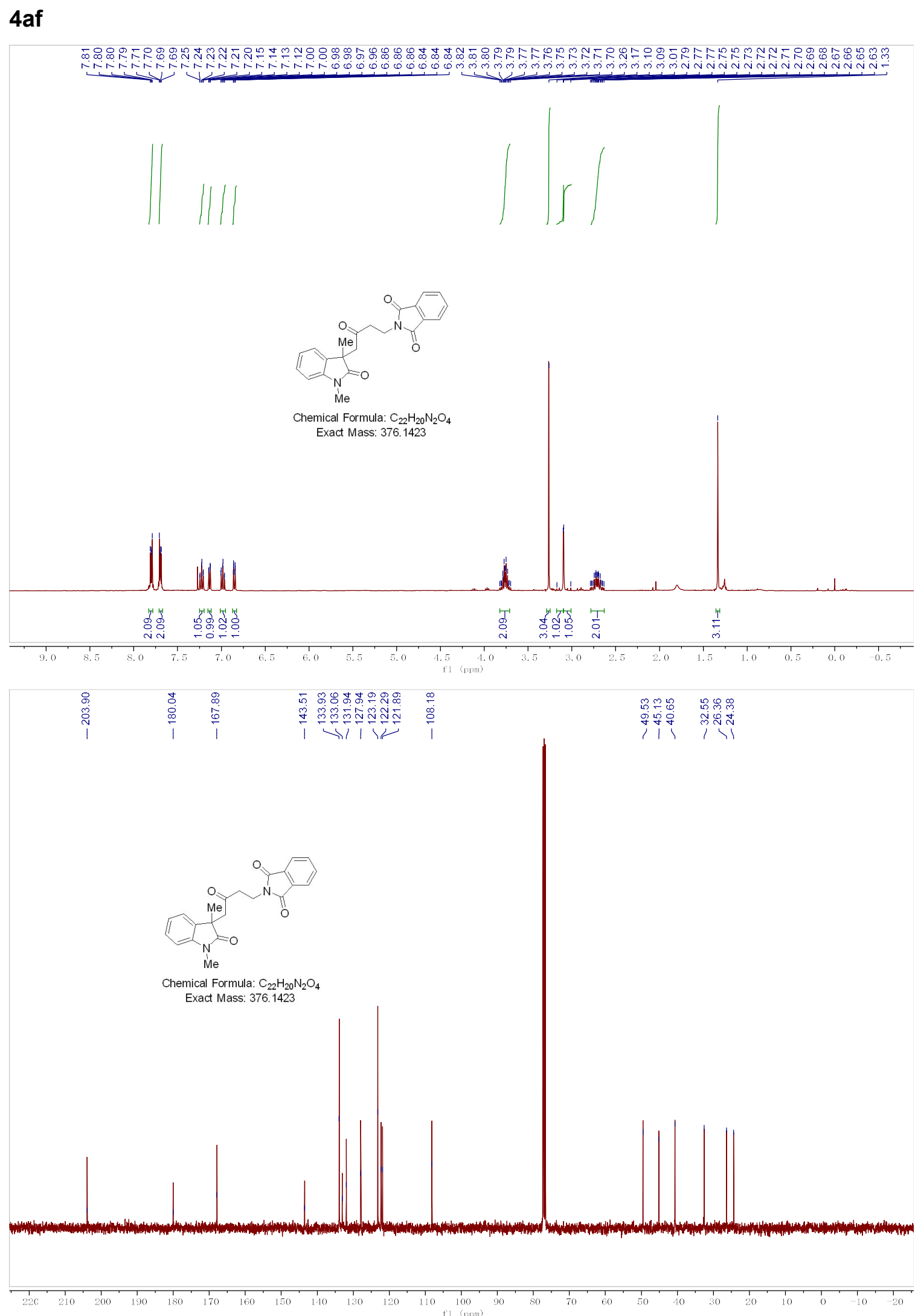
4ag

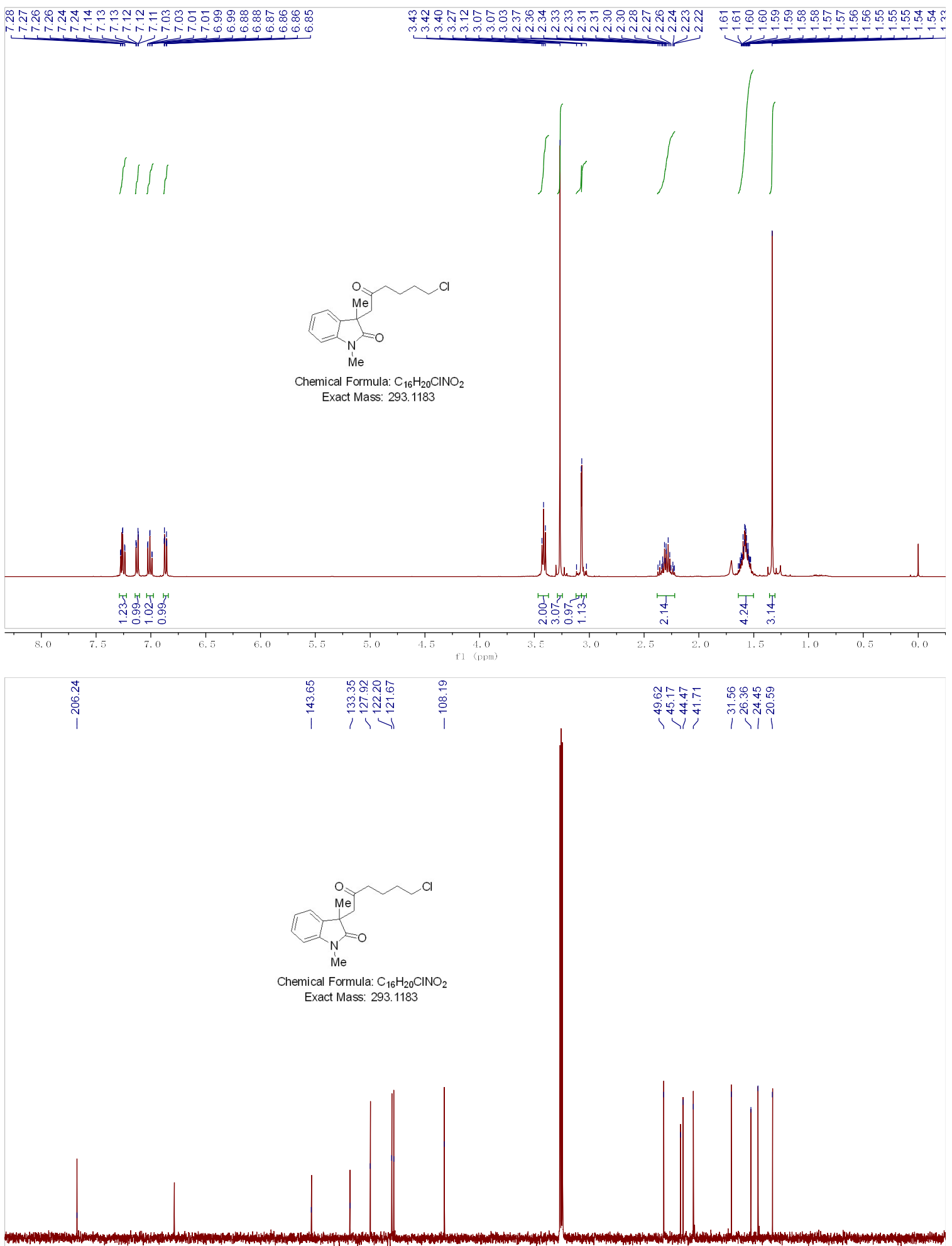

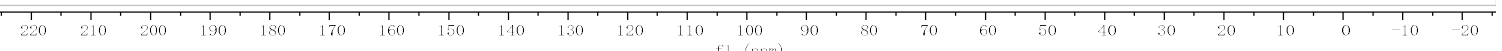


4ah

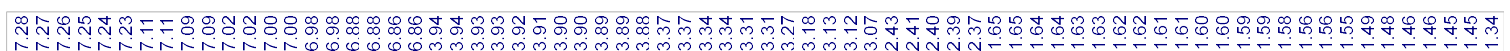

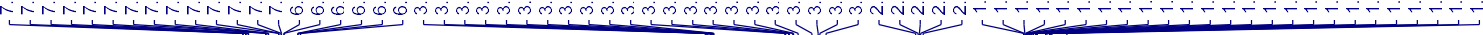
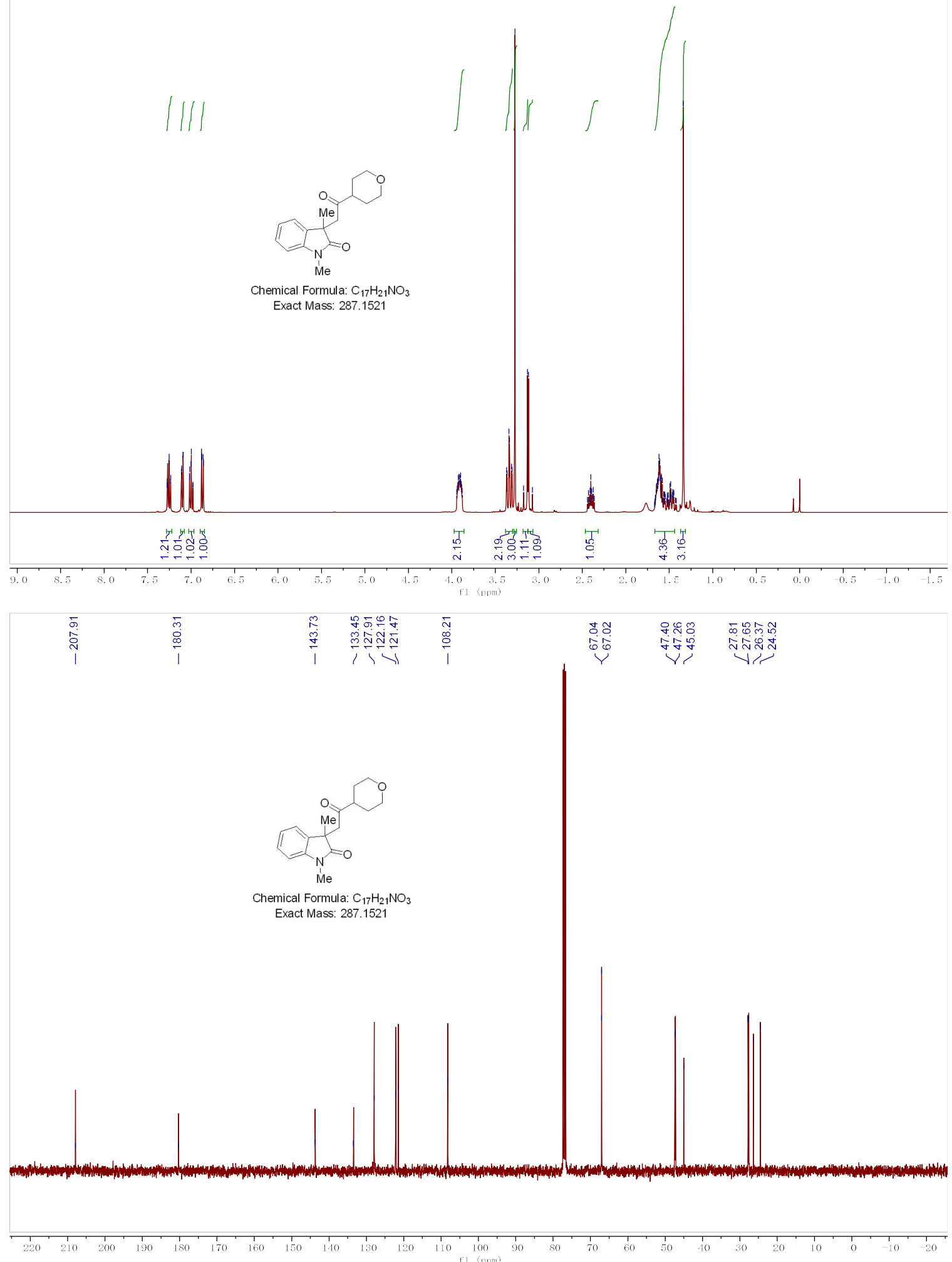
4ai

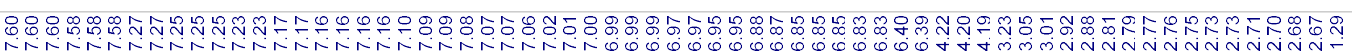

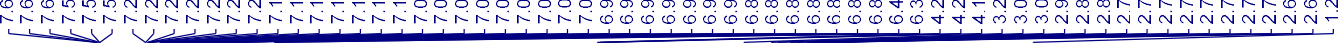

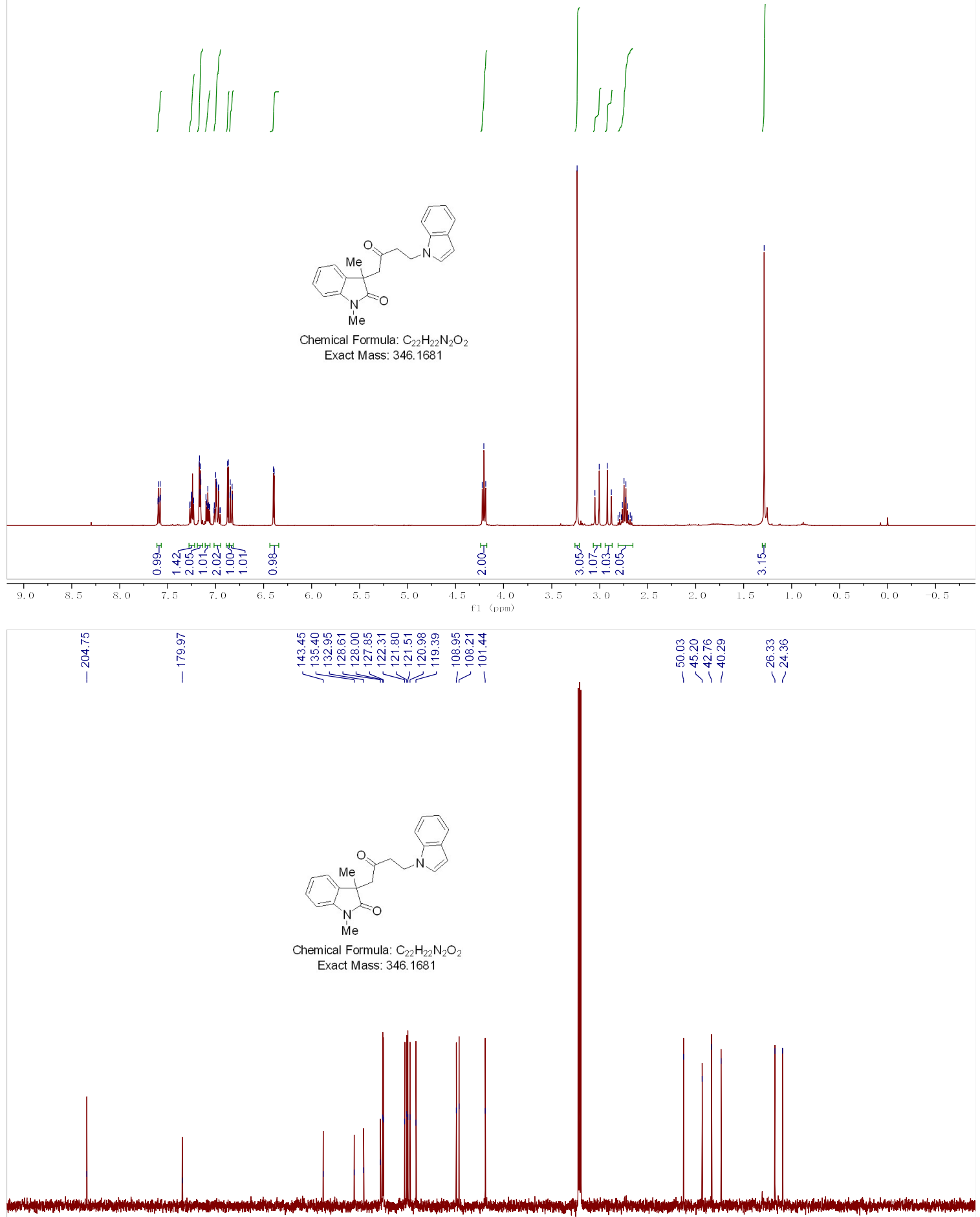

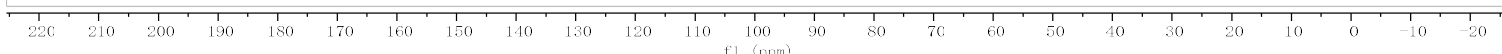


4aj

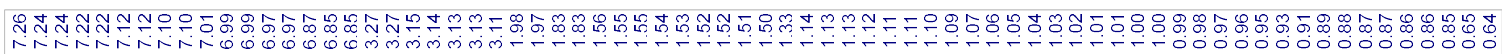

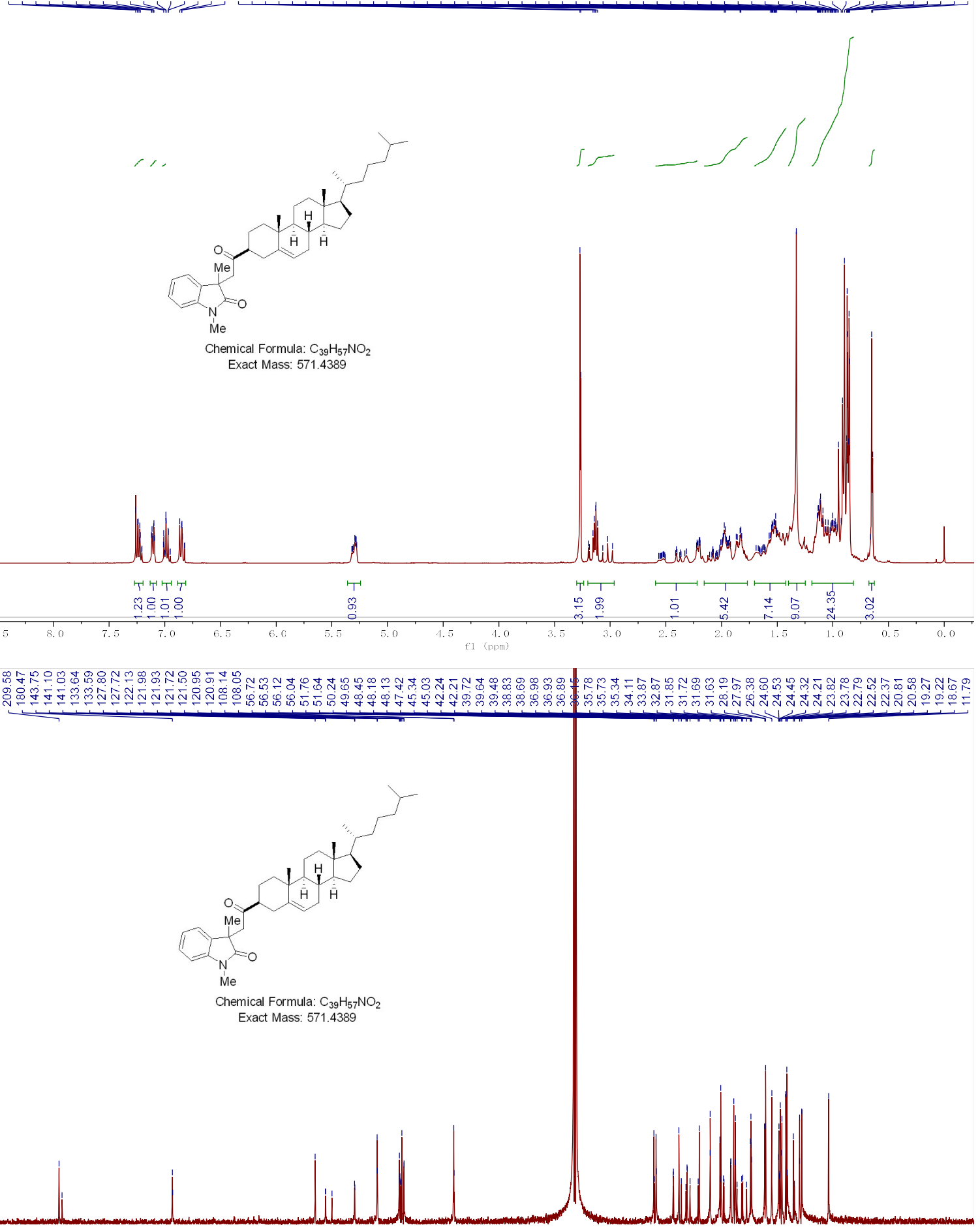

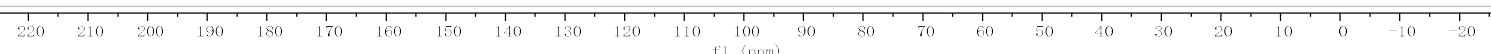




\section{4ak}

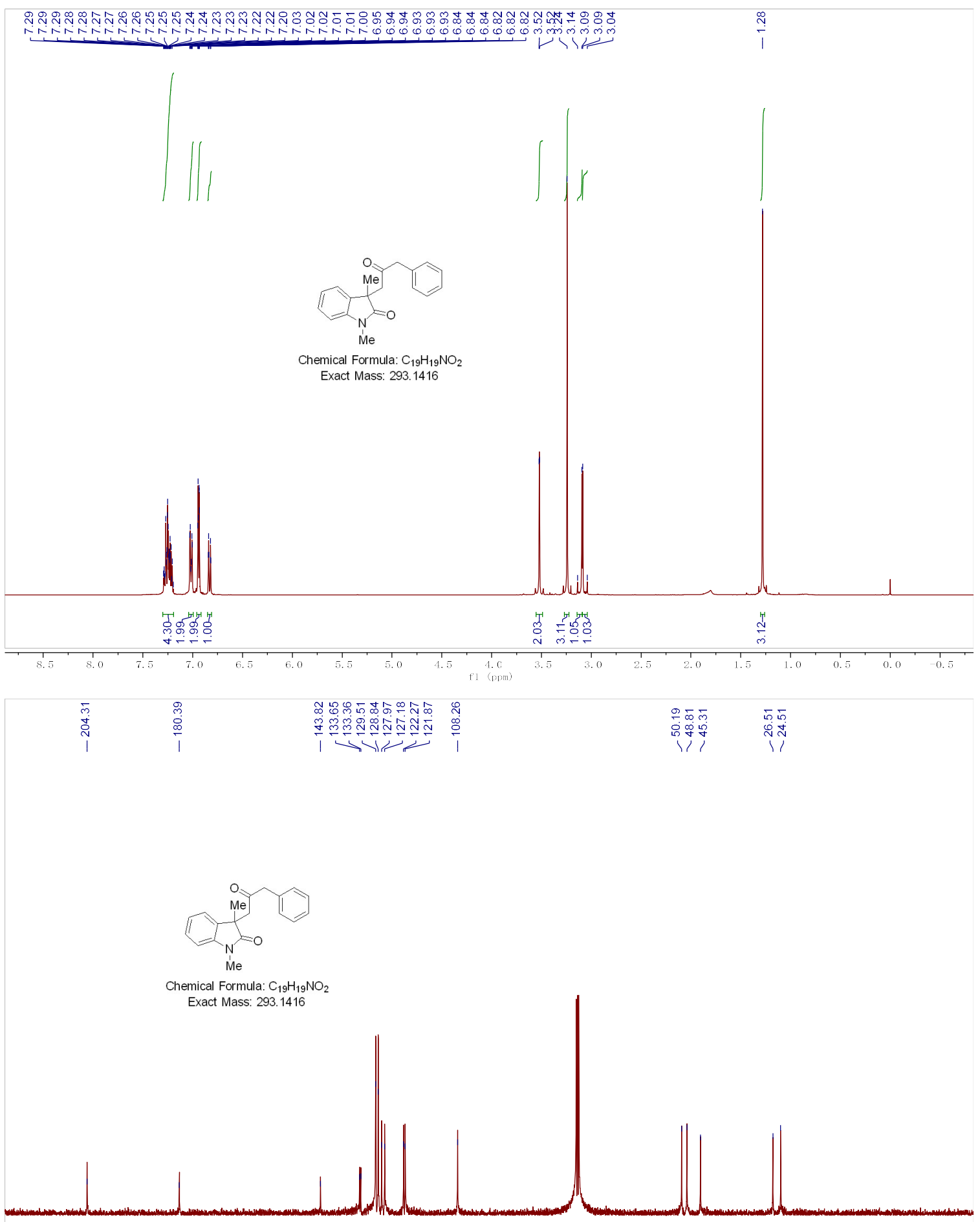

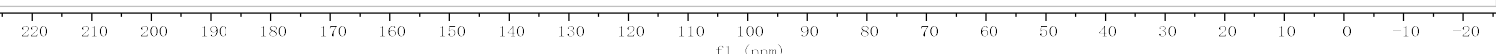




\section{4al}

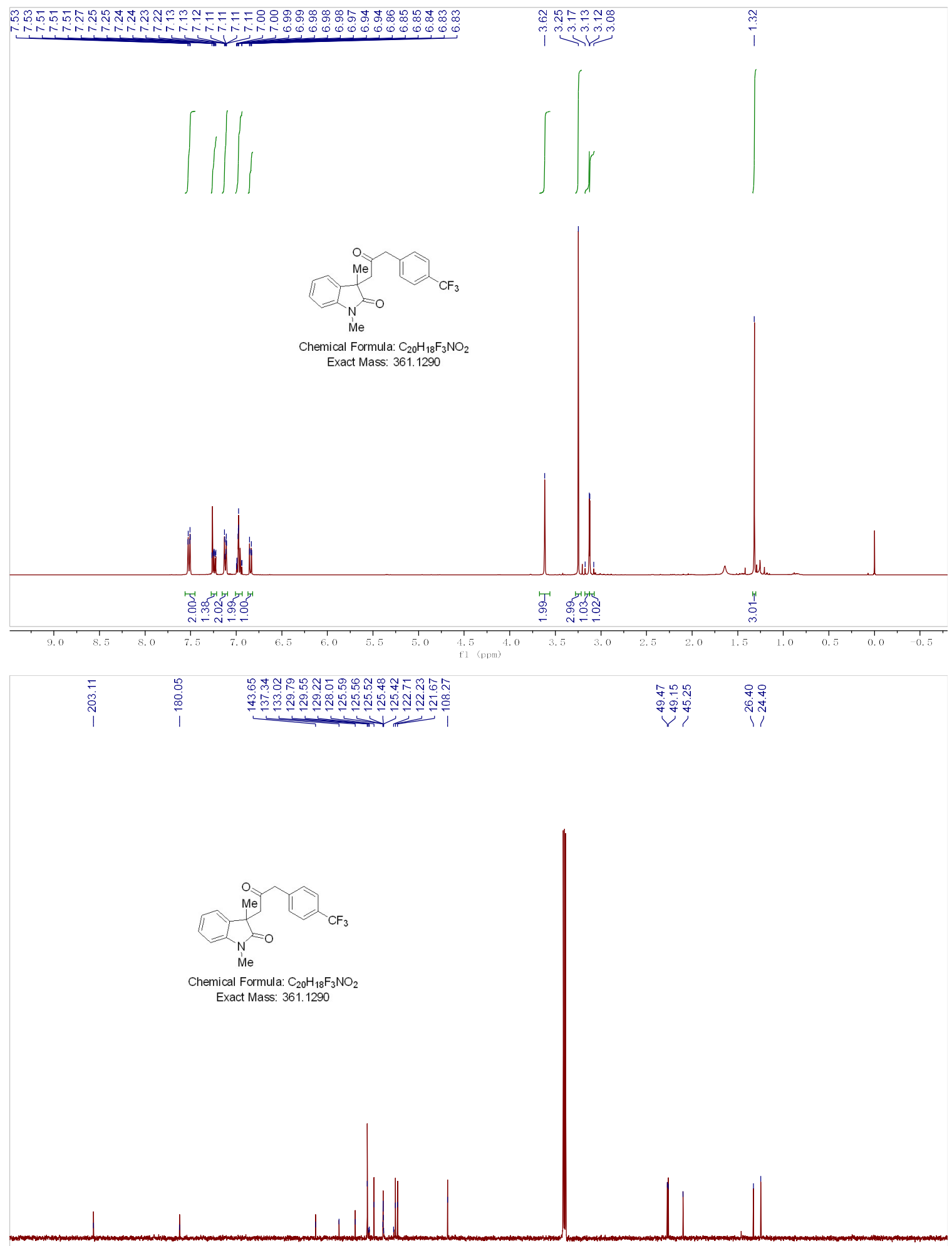

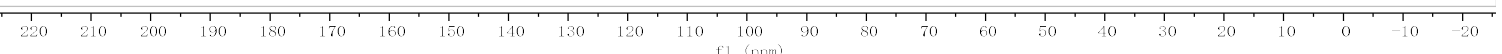



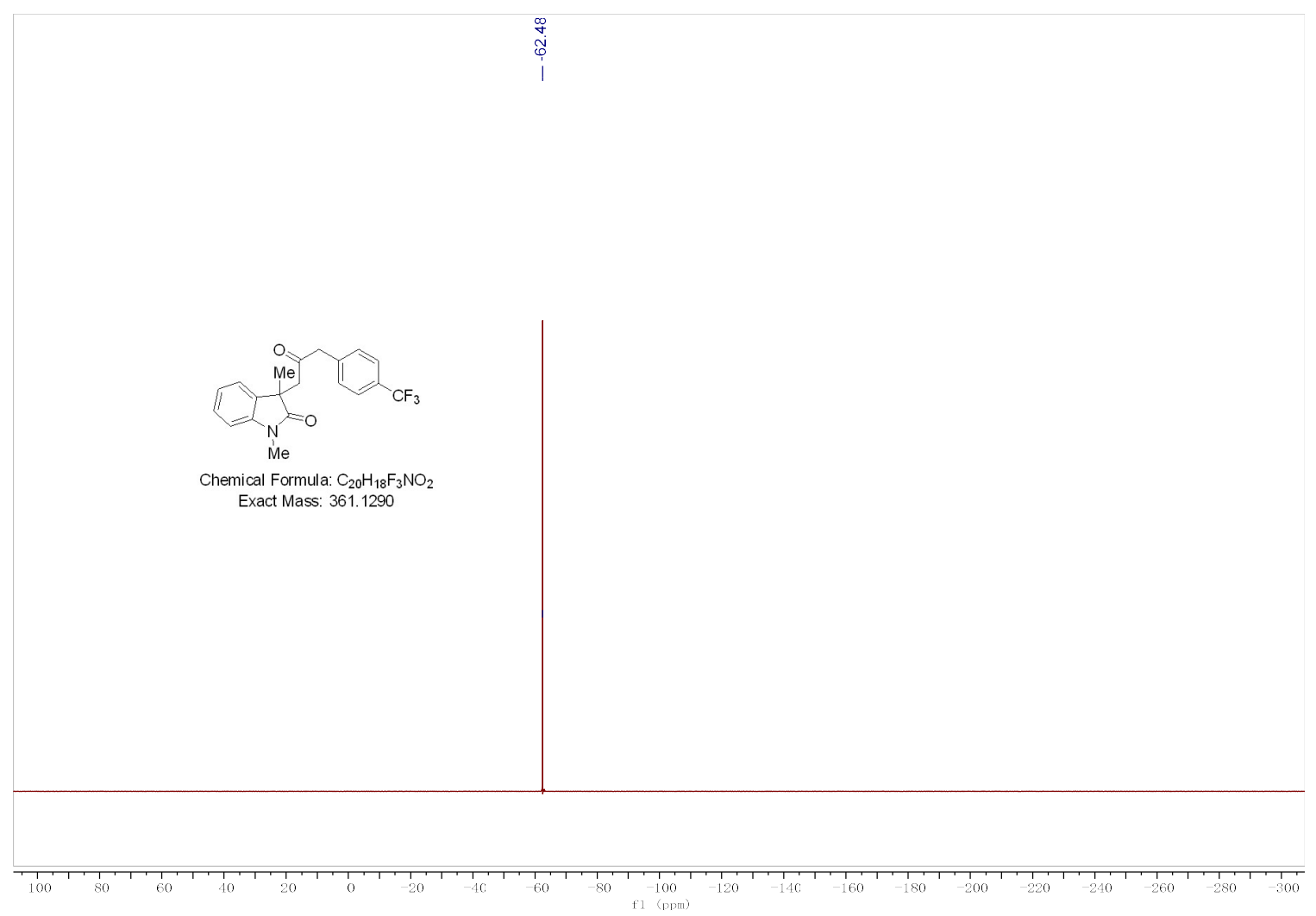

\section{4am}

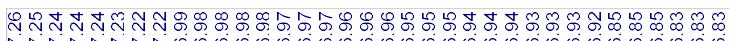

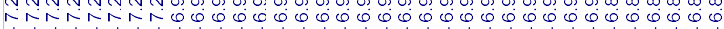

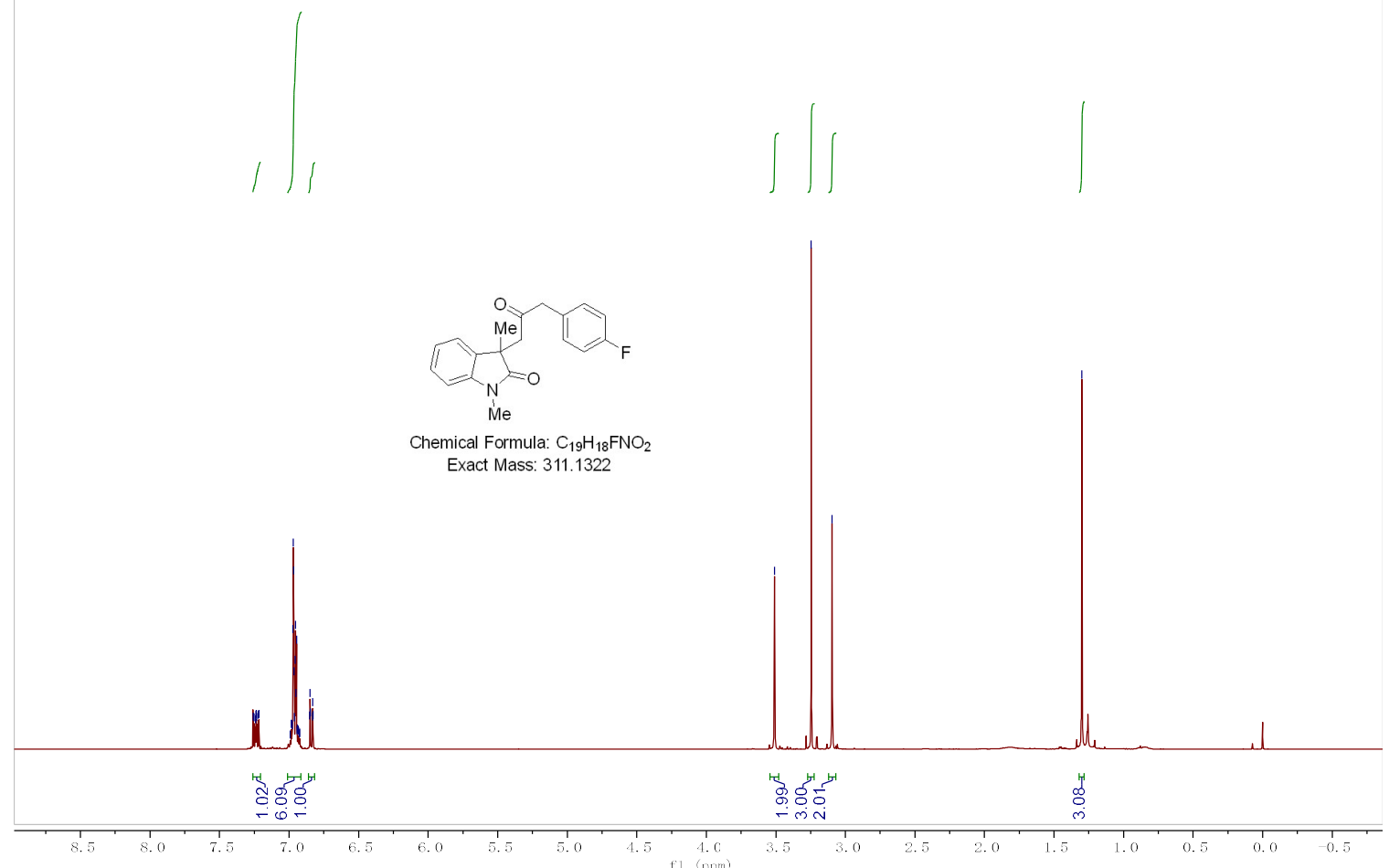

\section{Б5 $2 \frac{0}{\mathrm{~m}}$}



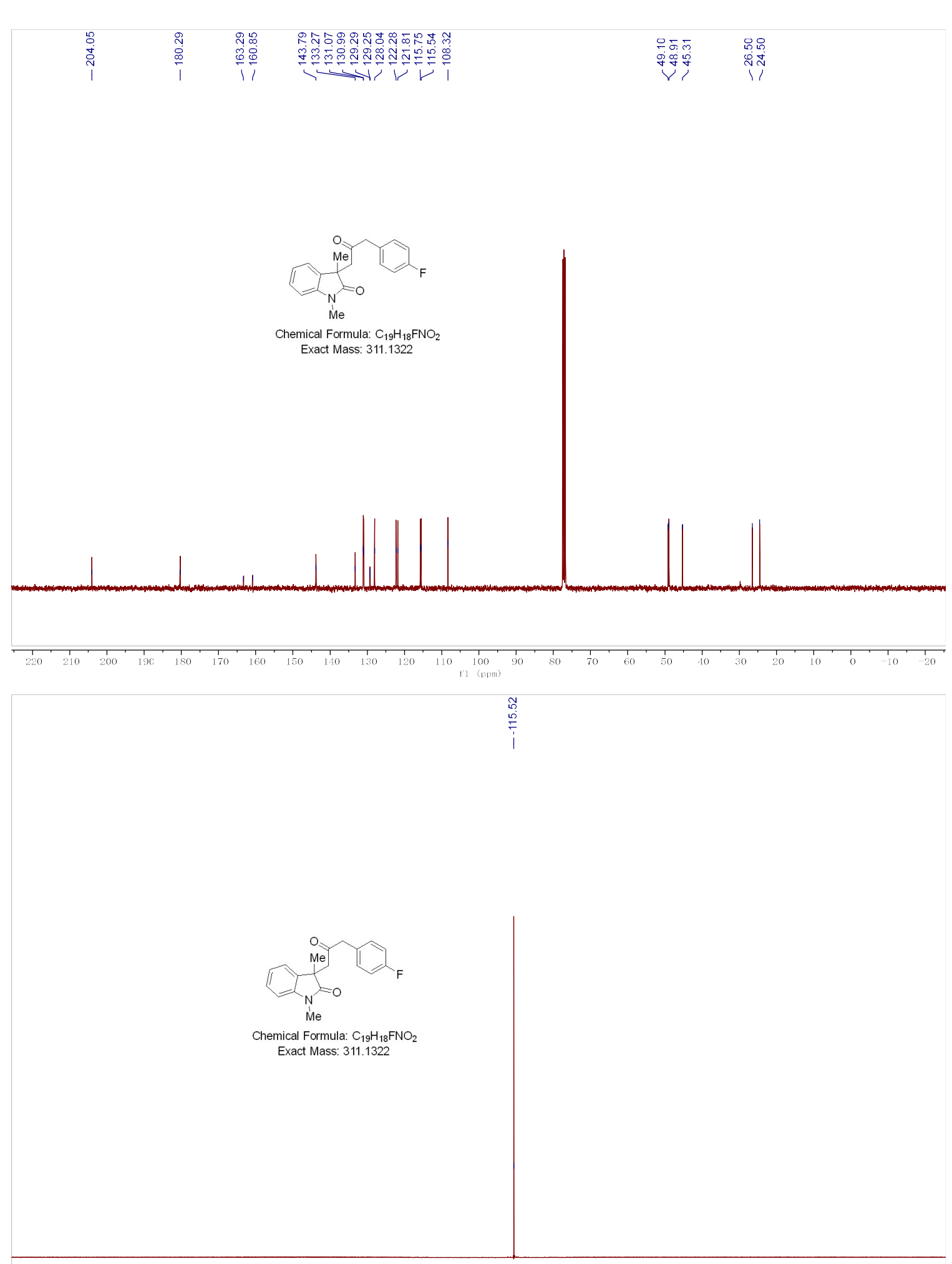

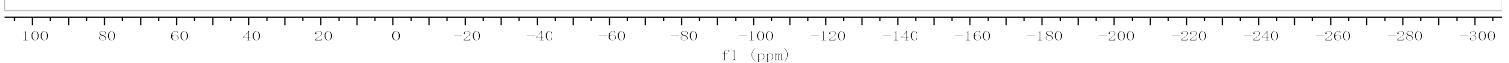




\section{4an}
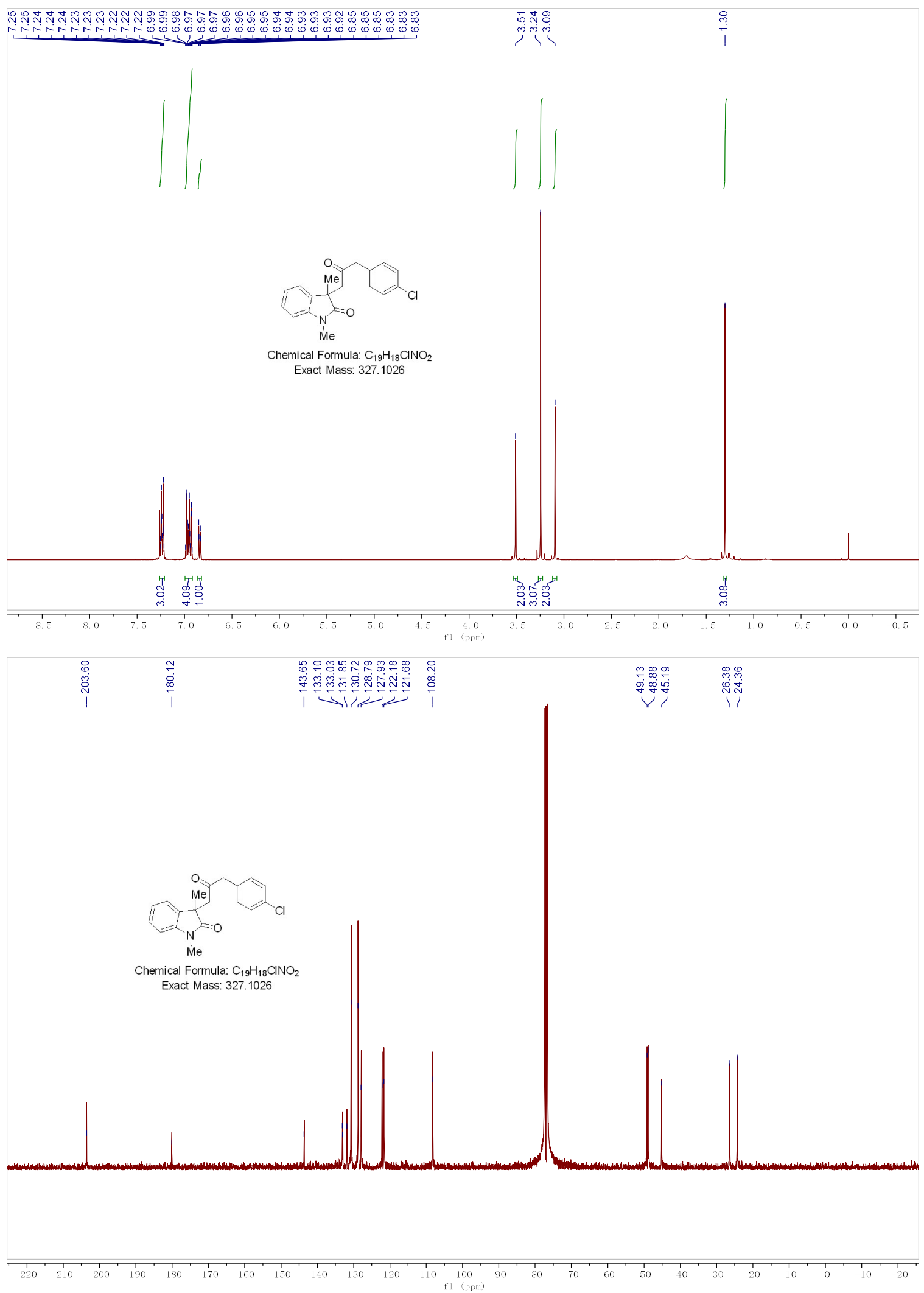


\section{4ao}

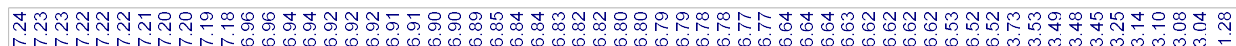

MATMATAT0000000000000000000000000000000000000mmmmmmmm
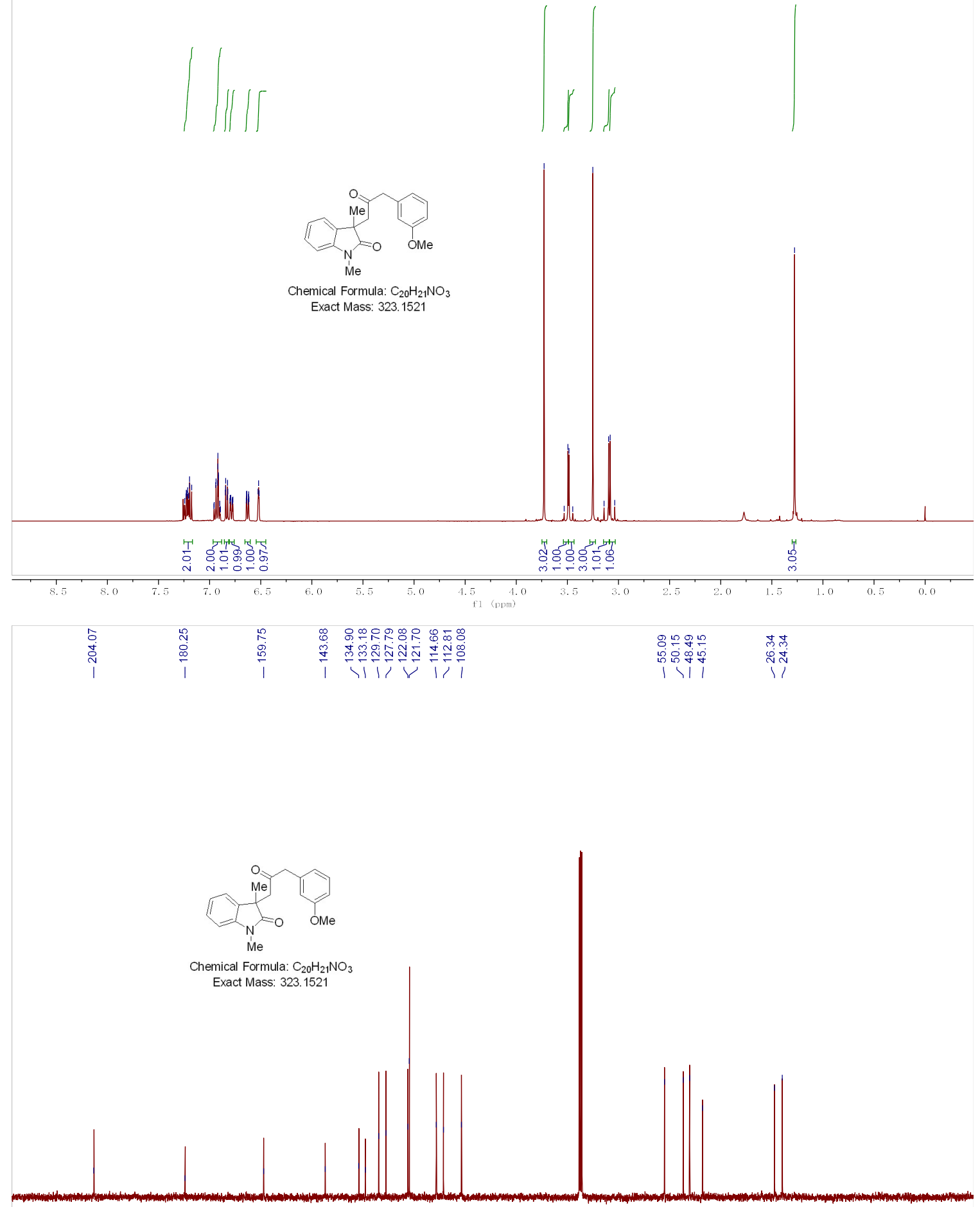


\section{6aa}

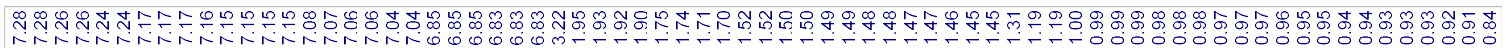
W

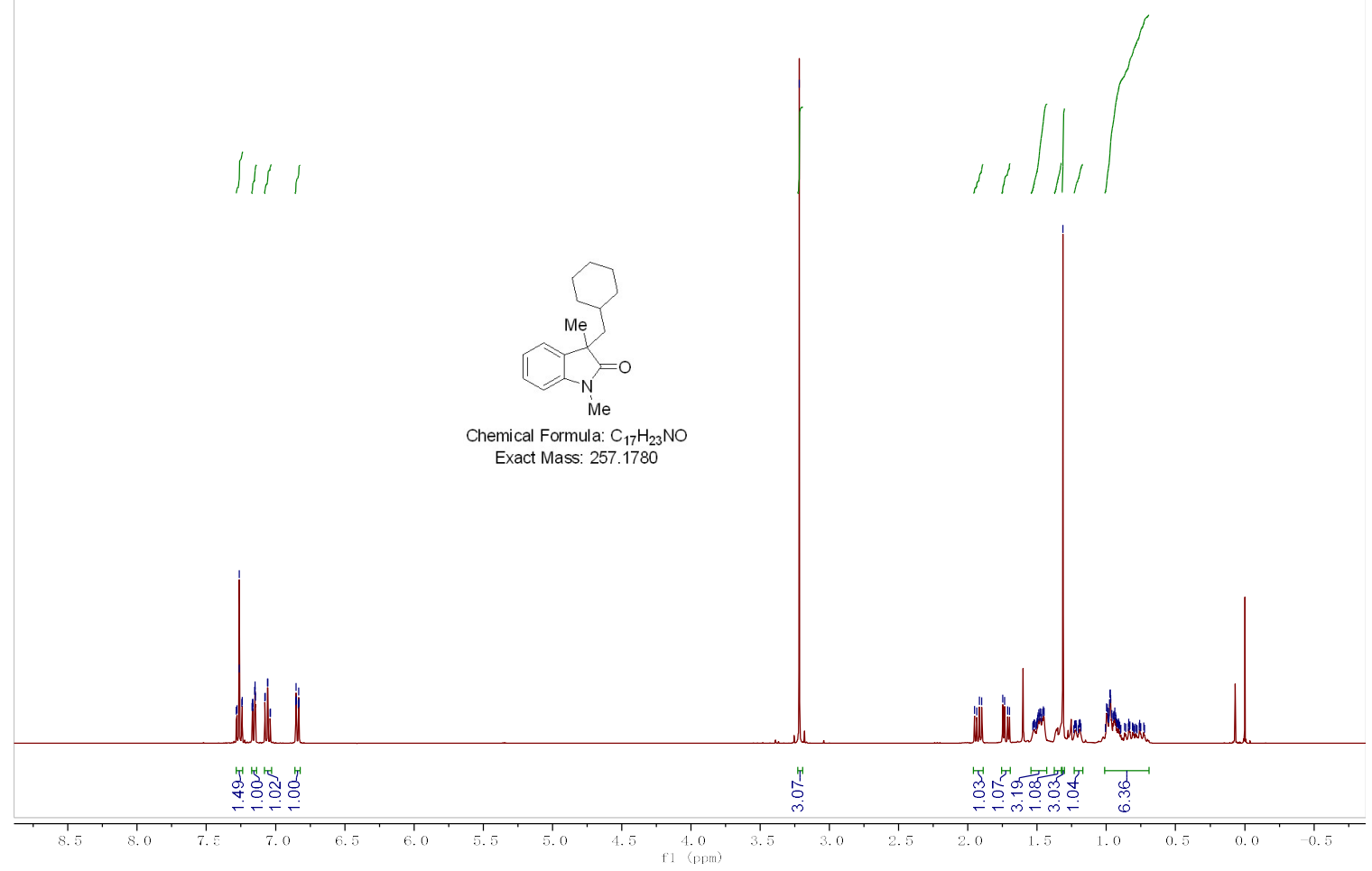




\section{References}

1. (a) Wang, H.; Guo, L.; Duan, X. Palladium-Catalyzed Alkylarylation of Acrylamides with Unactivated Alkyl Halides. J. Org. Chem. 2016, 81, 860. (b) Li, Z.; Zhang, Y.; Zhang, L.; Liu, Z. Free-Radical Cascade Alkylarylation of Alkenes with Simple Alkanes: Highly Efficient Access to Oxindoles via Selective $\left(\mathrm{sp}^{3}\right) \mathrm{C}-\mathrm{H}$ and $\left(\mathrm{sp}^{2}\right) \mathrm{C}-\mathrm{H}$ Bond Functionalization. Org. Lett. 2014, 16, 382. (c) Ling, A.; Zhang, L.; Tan, R.; Liu, Z. Molecular Oxygen-Promoted General and Site-Specific Alkylation with Organoboronic Acid. J. Org. Chem. 2018, 83, 14489.

2. Sakamoto, R.; Hiramaa, N.; Maruoka, K. The radical acylarylation of $\mathrm{N}$-arylacrylamides with aliphatic aldehydes using the photolysis of hypervalent iodine(III) reagents. Org. Biomol. Chem. 2018, 16, 5412. 